\title{
An Analysis of Testing Requirements for Fluoride Salt-Cooled High Temperature Reactor Components
}

November 2009

\author{
Prepared by \\ David E. Holcomb \\ Sacit M. Cetiner \\ George F. Flanagan \\ Fred J. Peretz \\ Graydon L. Yoder, Jr.
}




\section{DOCUMENT AVAILABILITY}

Reports produced after January 1, 1996, are generally available free via the U.S. Department of Energy (DOE) Information Bridge.

Web site http://www.osti.gov/bridge

Reports produced before January 1, 1996, may be purchased by members of the public from the following source.

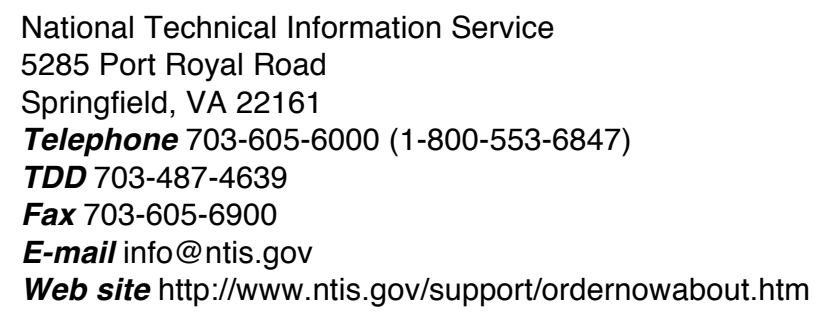

Reports are available to DOE employees, DOE contractors, Energy Technology Data Exchange (ETDE) representatives, and International Nuclear Information System (INIS) representatives from the following source.

Office of Scientific and Technical Information

P.O. Box 62

Oak Ridge, TN 37831

Telephone 865-576-8401

Fax 865-576-5728

E-mail reports@osti.gov

Web site http://www.osti.gov/contact.html

This report was prepared as an account of work sponsored by an agency of the United States Government. Neither the United States Government nor any agency thereof, nor any of their employees, makes any warranty, express or implied, or assumes any legal liability or responsibility for the accuracy, completeness, or usefulness of any information, apparatus, product, or process disclosed, or represents that its use would not infringe privately owned rights. Reference herein to any specific commercial product, process, or service by trade name, trademark, manufacturer, or otherwise, does not necessarily constitute or imply its endorsement, recommendation, or favoring by the United States Government or any agency thereof. The views and opinions of authors expressed herein do not necessarily state or reflect those of the United States Government or any agency thereof. 
Nuclear Science and Technology Division

\title{
AN ANALYSIS OF TESTING REQUIREMENTS FOR FLUORIDE SALT-COOLED HIGH TEMPERATURE REACTOR COMPONENTS
}

\author{
David E. Holcomb \\ Sacit M. Cetiner \\ George F. Flanagan \\ Fred J. Peretz \\ Graydon L. Yoder, Jr.
}

Date Published: November 2009

Prepared by

OAK RIDGE NATIONAL LABORATORY

Oak Ridge, Tennessee 37831-6283

managed by

UT-BATTELLE, LLC

for the

U.S. DEPARTMENT OF ENERGY

under contract DE-AC05-00OR22725 



\section{CONTENTS}

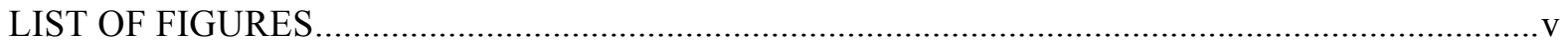

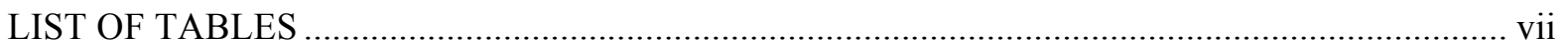

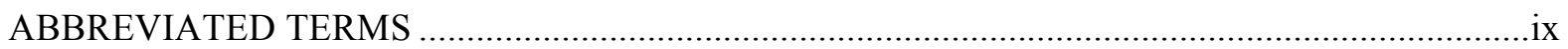

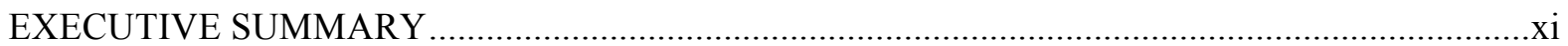

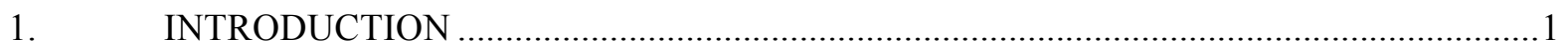

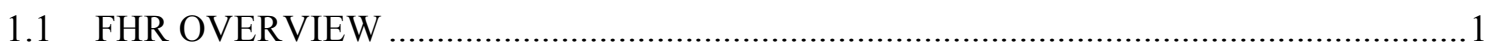

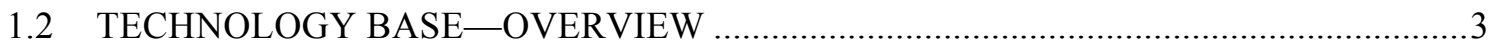

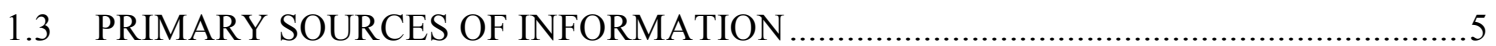

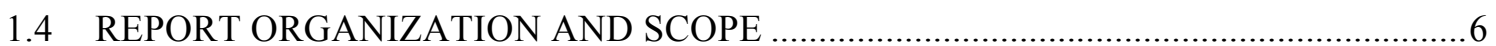

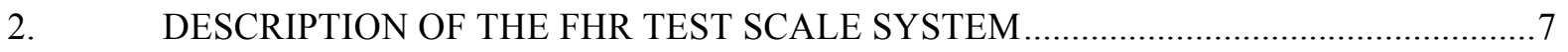

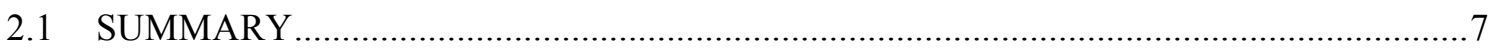

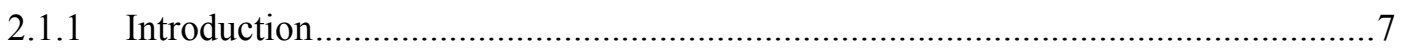

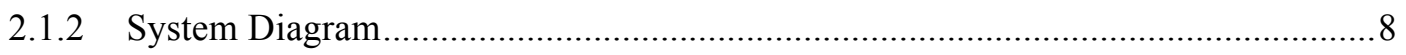

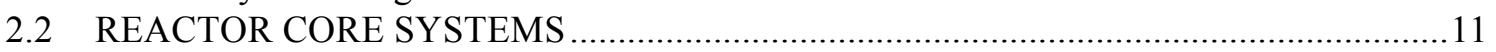

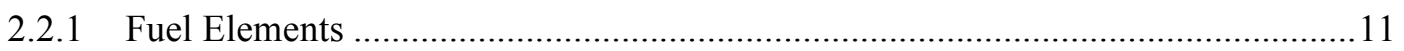

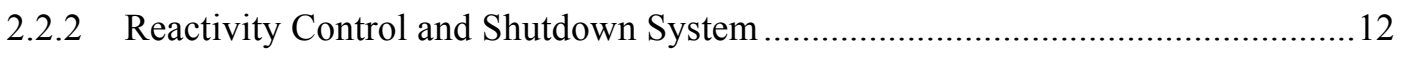

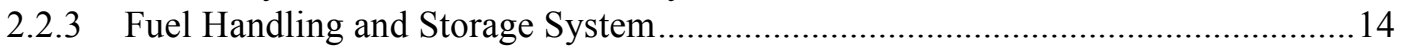

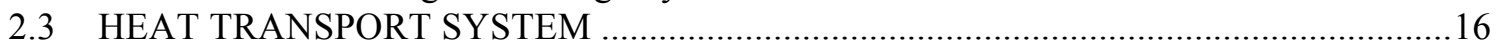

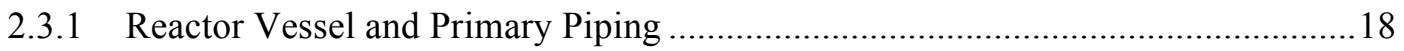

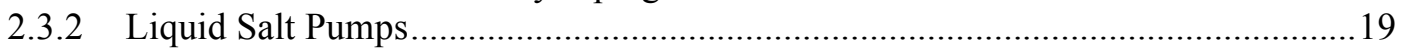

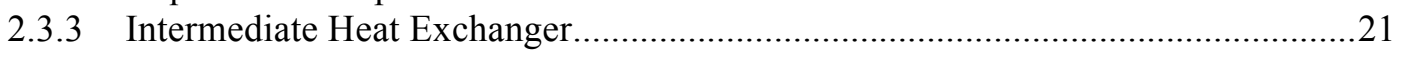

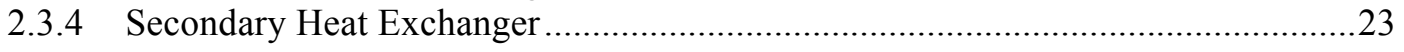

2.3.5 Salt Processing and Inventory Control System.....................................................23

2.3.6 Cover Gas Chemistry and Pressure Control System .............................................24

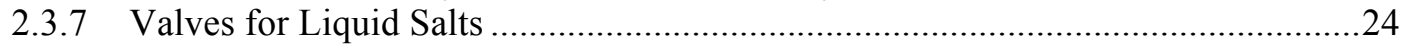

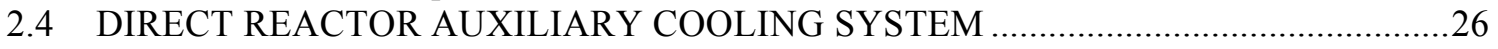

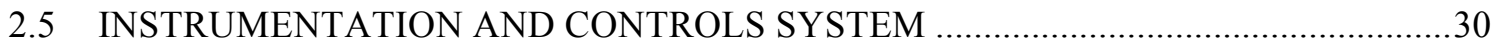

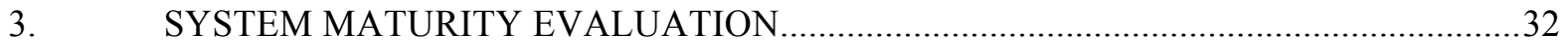

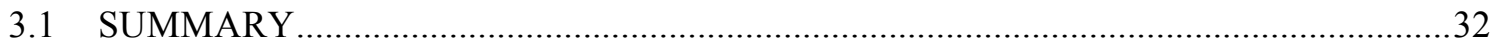

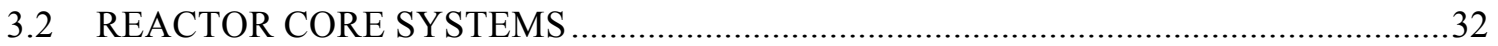

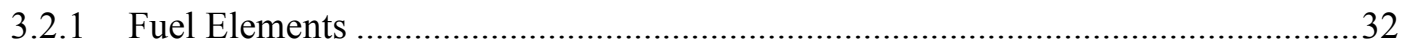

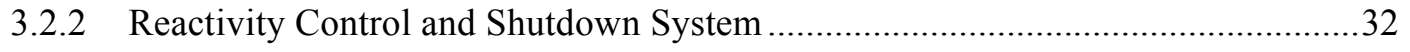

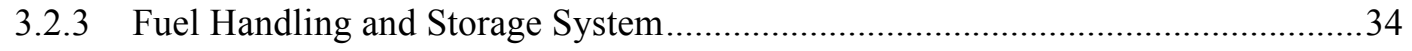

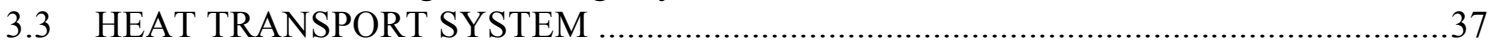

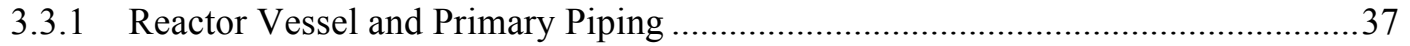

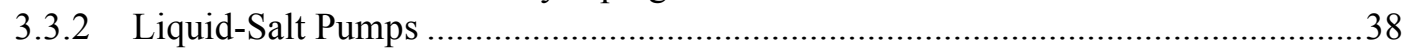

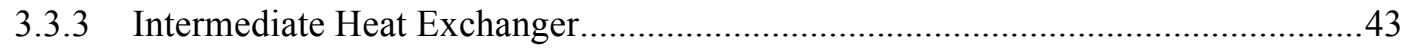

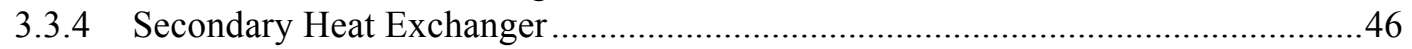

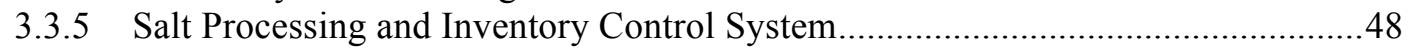

3.3.6 Cover Gas Chemistry and Pressure Control System ............................................49

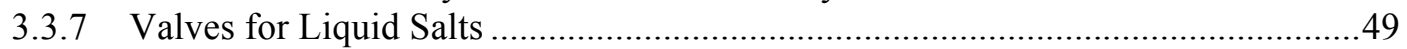

3.4 DIRECT REACTOR AUXILIARY COOLING SYSTEM .............................................51

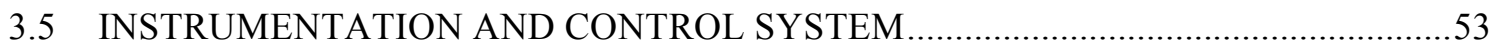

4. IDENTIFICATION OF COMPONENT TEST REQUIREMENTS ….............................56

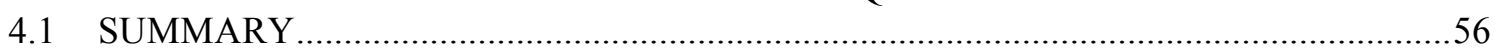

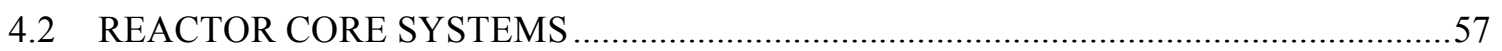




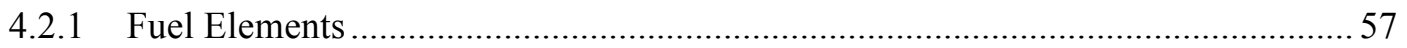

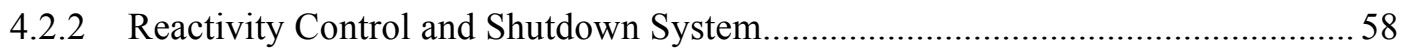

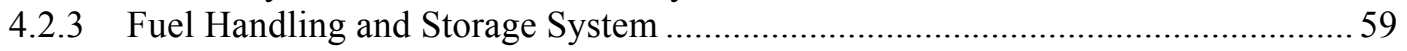

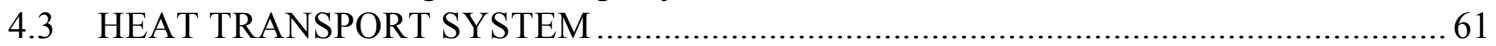

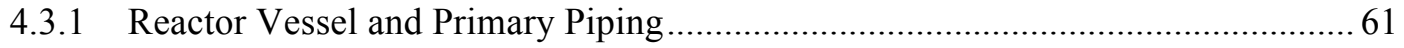

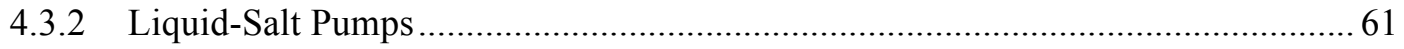

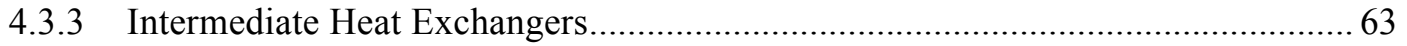

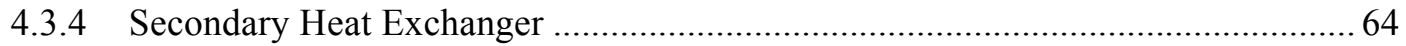

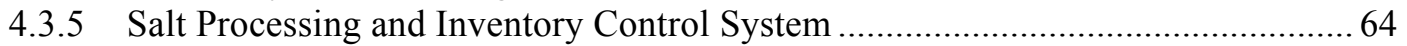

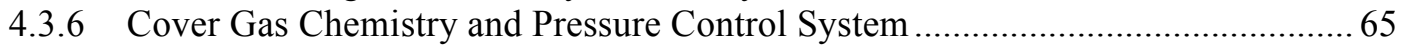

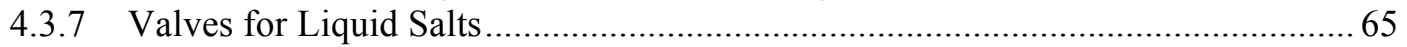

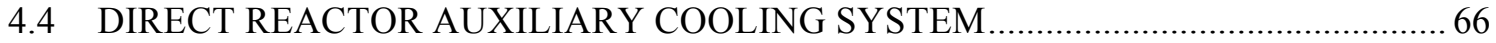

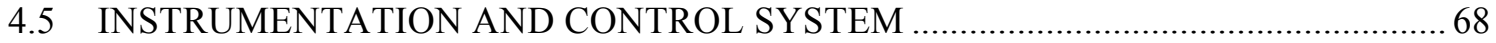

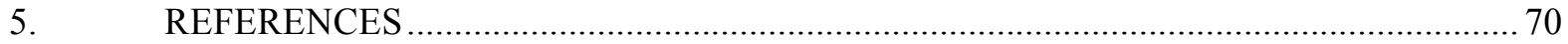




\section{LIST OF FIGURES}

Figure $\quad$ Page

Fig. 1. Conceptual drawing of a pebble bed FHR and power generation cycle.................. 2

Fig. 2. The MSRE as seen from above....................................................................... 4

Fig. 3. Overall FHR-TS primary heat transfer flow ..................................................... 8

Fig. 4. FHR-TS process flow block diagram ............................................................ 9

Fig. 5. Photograph of UCB FHR-TS core model ......................................................... 11

Fig. 6. FHR (a) fuel pebble, fuel pebble cross section, and coated particle;

(b) enlarged view of a coated particle .......................................................... 12

Fig. 7. Cross section of a high temperature reactor shutdown absorber stack.................. 13

Fig. 8. Poisoned salt secondary shutdown system ................................................. 14

Fig. 9. A conceptual drawing of fuel pebble handling in an FHR ................................ 15

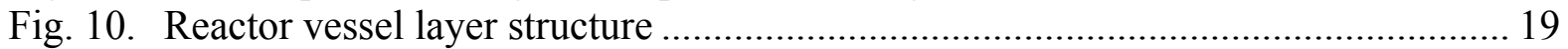

Fig. 11. Cross-section of the MSRE fuel salt pump .................................................... 20

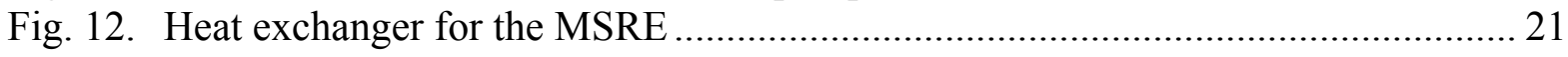

Fig. 13. Freeze valve design from the MSRE ............................................................. 25

Fig. 14. Schematic drawing of DRACS and its interface with the Primary Heat

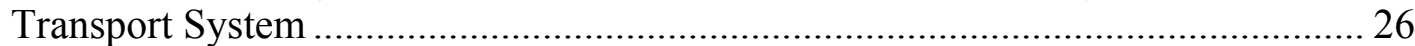

Fig. 15. Simplified schematic of floating ball type fluid diode: (a) low flow-resistance condition, (b) high flow-resistance condition ................................................. 28

Fig. 16. Vortex type fluid diode: (a) reverse flow (high resistance), (b) forward flow

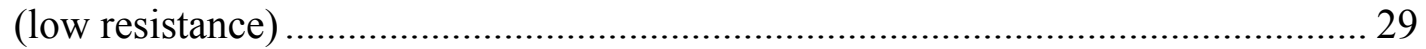

Fig. 17. Fluidic diode design based on guide blades................................................... 29

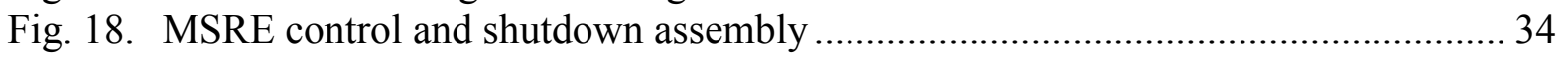

Fig. 19. Test of fuel pebble handling proposed for the PB-AHTR ................................... 36

Fig. 20. Photograph of the PREX-1 pebble test facility ................................................ 36

Fig. 21. Long-shaft liquid-salt pump with one liquid-salt lubricated bearing..................... 39

Fig. 22. Primary heat exchanger subassemblies of the MSRE....................................... 43

Fig. 23. Tube-to-tube sheet joint in the MSRE primary heat exchanger ........................... 46

Fig. 24. Early salt-to-gas heat exchanger design.......................................................... 47

Fig. 25. Shutdown cooler bayonet heat exchanger in EBR-II............................................ 52 



\section{LIST OF TABLES}

Table

Page

Table 1. FHR-TS general design parameters as compared to the MSRE .........................................10

Table 2. Primary Heat Transport System baseline parameters ....................................................... 16

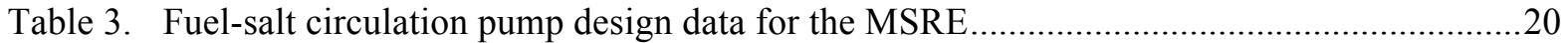

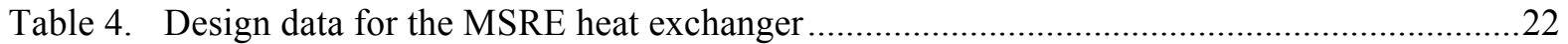

Table 5. Characteristics and operation time for ORNL salt and liquid metal pumps ........................40

Table 6. Comparison of major operating parameters between the MSRE primary heat exchanger and the FHR-TS baseline design Intermediate Heat Exchanger .........................44

Table 7. Summary of FHR-TS near-term component testing requirements .....................................69 



\section{ABBREVIATED TERMS}

$\begin{array}{ll}\text { AHTR } & \text { Advanced High Temperature Reactor } \\ \text { ANP } & \text { Aircraft Nuclear Propulsion } \\ \text { ARE } & \text { Aircraft Reactor Experiment } \\ \text { ASME } & \text { American Society of Mechanical Engineers } \\ \text { CAD } & \text { computer-aided design } \\ \text { CANDU } & \text { Canada deuterium uranium reactor } \\ \text { CTF } & \text { component test facility } \\ \text { DHX } & \text { DRACS heat exchanger } \\ \text { DRACS } & \text { direct reactor auxiliary cooling system } \\ \text { EBR-II } & \text { Experimental Breeder Reactor II } \\ \text { EPR } & \text { European pressurized reactor } \\ \text { FEM } & \text { finite element method } \\ \text { FHR } & \text { fluoride salt-cooled high temperature reactor } \\ \text { FHR-TS } & \text { FHR test scale } \\ \text { FHSS } & \text { fuel handling and storage system } \\ \text { FPHE } & \text { formed plate heat exchanger } \\ \text { H'X } & \text { hybrid heat exchanger } \\ \text { HSI } & \text { human-system interface } \\ \text { HTGR } & \text { high temperature gas-cooled reactor } \\ \text { I\&C } & \text { instrumentation and controls } \\ \text { IHTS } & \text { intermediate heat transport system } \\ \text { IHX } & \text { intermediate heat exchanger } \\ \text { LMFBR } & \text { liquid-metal fast breeder reactor } \\ \text { LMTD } & \text { log-mean temperature difference } \\ \text { LWR } & \text { light water reactor } \\ \text { MSBE } & \text { Molten Salt Breeder Experiment } \\ \text { MSR } & \text { molten salt reactor } \\ \text { MSRE } & \text { Molten Salt Reactor Experiment } \\ \text { NDHX } & \text { natural draft heat exchanger } \\ \text { NGNP } & \text { Next Generation Nuclear Plant } \\ \text { PBMR } & \text { pebble bed modular reactor } \\ \text { PCS } & \text { power conversion system } \\ \text { PHTS } & \text { primary heat transport system } \\ \text { PICS } & \text { processing and inventory control subsystem } \\ \text { PREX } & \text { Pebble Recirculation Experiment } \\ \text { R\&D } & \text { research and development } \\ \text { RV } & \text { reactor vessel } \\ \text { SAFR } & \text { sodium advanced fast reactor } \\ \text { SCS } & \text { shutdown cooling system } \\ \text { UCB } & \text { University of California-Berkeley } \\ & \\ \text { HW }\end{array}$





\section{EXECUTIVE SUMMARY}

Nuclear power plant components must function reliably both individually and as integrated systems. Testing the performance of the plant components and systems is critical to acquiring confidence that a reactor design will function as intended. While much of the technology of the prior molten salt reactor program and the current high temperature gas reactor program can be directly applied to fluoride salt-cooled reactors, the testing requirements for the new fluoride salt-cooled high temperature reactor class have not previously been evaluated.

Fluoride salt-cooled high temperature reactors (FHRs) are an emerging reactor class. The essential features of FHR class reactors are fluoride salt cooling, high temperature heat production, coated particle fuel, and a Brayton energy conversion cycle. No complete FHR class design has yet been developed. The first FHR will almost of necessity be a test-scale reactor to validate the system attributes before proceeding to larger scale systems. Component test planning is therefore focused on supporting a smaller size $[<20 \mathrm{MW}(\mathrm{t})]$ reactor.

This report seeks to identify and describe the reactor component performance and reliability requirements, determine what information is necessary to provide assurance that components will adequately achieve the requirements, and then provide guidance on how the required performance information can efficiently be obtained. The report focus is bounded at the interface between and the primary coolant salt and the fuel and the gas supply and return to the Brayton cycle power conversion system. The analysis is also limited to component level testing and does not address system level testing issues. Further, the report is oriented as a bottom-up testing requirements analysis as opposed to a having a top-down facility description focus.

The information and analysis presented in this report represent an amalgamation of several information sources. The reactor conceptual design is primarily derived from the most recent University of California at Berkeley FHR-16 test reactor design overview documents and the slightly older ORNL prismatic block fuel Advanced High Temperature Reactor (AHTR) development program reports. Much of the component design information was obtained from the extensive documentation set of the prior molten salt reactor program. Additionally, significant material status information was obtained from the fluoride salt-cooled reactor materials technology plan that is currently in preparation as well as the present day high temperature gas reactor design description documents.

Component test planning is part of a larger FHR development effort. Materials evaluation and development, separate and integral effect testing to support safety evaluation, fuel development and performance testing, and creation of a complete conceptual reactor design are the additional steps required to advance FHR systems. Demonstration of the key performance and safety advantages of the reactor class, system economic analysis, and reestablishment of lithium isotopic enrichment are also central to advancing the development of FHRs as a preferred power generation option.

When a completed test reactor design becomes available, the design assumptions embodied in this report will need to be revisited. The design parameters should only be considered placeholders until an endorsed test reactor design is developed. While the overall plant design will evolve with development effort, component designs and requirements are to a large degree independent of the plant design. All candidate fluoride salt coolants have similar thermal and hydraulic properties, and the same amount of decay heat must be rejected to the environment independent of fuel configuration. 
The power plant design parameters provided in this report inherently reflect technology development rate assumptions. For example as more advanced fluoride salt compatible materials become available the core outlet temperature and therefore the plant thermal efficiency will rise, yet a conceptual design being completed today would be restricted to currently available materials. The report technology discussions reflect this ambiguity describing systems both with available technology as well as incorporating technologies that have a high probability of becoming available over the next few years.

The most pressing system technology testing need is demonstration of reactor auxiliary cooling. Many of FHR desirable properties are critically dependent on the ability to reject decay heat to the local environment through natural circulation without fuel damage under loss of forced circulation conditions. The direct reactor auxiliary cooling system (DRACS) performance, while derived from earlier liquid metal reactor experience, has not previously been demonstrated experimentally. Of nearly equal importance is performance demonstration of the secondary heat exchanger that transfers the reactor's energy from a low-pressure salt to a high-pressure fluid. This heat transfer component did not exist in the molten salt reactor program. This secondary heat exchanger has challenging performance and environmental tolerance requirements, and its operation is central to the desirability of FHRs. Finally, simply reestablishing the technologies to handle, purify, and continuously operate reactor-scale molten salt components would greatly enhance the acceptability of liquid salt reactor designs.

A variety of specialized testing stands and heat transfer loops are needed to support system design, reliability evaluation, and safety demonstrations. The testing needs will evolve from design models to surrogate materials test stands to scaled prototypic materials component testing to integrated system testing as the reactor design progresses. In the near term only moderate-size equipment is required, with roughly one megawatt being the upper end of the test system power and a few tens of kilograms per minute maximum coolant flow rate. Only for validation and acceptance testing of full-scale test reactor systems will larger facilities be required. While testing using beryllium bearing salts will be necessary for validating the salt lubrication and corrosion properties, the thermal and hydraulic properties of candidate fluoride salts are sufficiently similar and described by known scaling laws so as to permit key tests to be performed with low toxicity fluoride salts.

Quality assurance is a principal requirement for any testing used in making nuclear safety or design decisions. While final technology maturity demonstration will require test facilities at a representative scale, initial testing can be performed by multiple collaborating organizations with small-scale facilities. Creating accessible fluoride salt test systems backed by a nuclear-grade quality assurance program at national labs and industry, with universities partnering in their operation to support design innovation, appears to be an efficient way to develop the required system and component performance, reliability, and safety information. 


\section{INTRODUCTION}

The purpose of this report is to establish what component testing would be required for a fluoride saltcooled high temperature test reactor and how the testing can most efficiently be performed to provide reasonable assurance of component performance and reliability. While the FHR test reactor will be of similar size as the Molten Salt Reactor Experiment (MSRE) that operated at ORNL from 1965 to 1969 and can benefit from the extensive experience gained from the MSRE, a modern component test facility is required to test FHR-specific components and to regain experience and capability which has atrophied since the end of the molten salt reactor program in 1975.

The FHR concept introduces several innovative reactor system components, which are not commonly employed in reactor design. The testing requirements evaluation has been divided into four steps.

1. Identify the FHR component safety, reliability, and performance requirements.

2. Determine the information necessary to provide reasonable assurance that the components will satisfy the requirements.

3. Develop a methodology to efficiently acquire the required information for each component and for the integrated operation of components as a system.

4. Establish component test facility $(\mathrm{CTF})$ requirements necessary to acquire the component performance data.

The component testing for any FHR class reactor would have large degrees of similarity. More definitive understanding of the specific science and technology issues is gained by analyzing a particular system. The FHR reactor concept currently under most active development is the pebble bed FHR-16 currently being investigated by the University of California at Berkeley (UCB). This report focuses baseline modeling and simulation on a similar test-scale reactor both because more extensive use can be made of the earlier MSRE data and because a test reactor provides the most practical route to demonstrate the advantageous features of an FHR.

The liquid cooling nature of an FHR makes matching key system parameters (power density, temperatures, flow velocity, etc.) between test- and commercial-scale systems feasible. As with light water reactors (LWRs), FHR operational and safety characteristics scale well from test-scale reactors to commercial-scale systems. Thus a flexibly designed test reactor would provide a science and technology platform to systematically research, develop, and demonstrate the technical potential of FHRs.

For planning purposes a pebble bed FHR test-scale reactor has been selected as the model system for testing requirements analysis. As no complete design yet exists for this reactor, modeling estimates represent an amalgam of the UCB FHR-16 design information, the prior molten salt reactor program design documents, and the earlier ORNL prismatic fuel Advanced High Temperature Reactor (AHTR) design along with engineering judgment as to how an integrated system would need to be configured. Use of a pebble bed FHR in this report does not imply that this reactor design is superior to or has been selected over other possible FHR configurations for next-stage development.

\subsection{FHR OVERVIEW}

FHRs are a candidate successor reactor class to LWRs. The acronym FHR is used here to represent the class of reactors that feature fluoride salt cooling, high temperature heat production, coated particle fuel, and a Brayton power generation cycle. These defining elements of FHRs provide the potential to markedly improve upon the already desirable attributes of LWRs, thus providing the potential for 
transformational improvement in energy production. An FHR reactor core consists of coated particle fuel embedded within graphite fuel elements. Graphite reflectors provide additional moderation and core structure. Heat is removed from the reactor core by a primary liquid fluoride salt coolant. The reactor's heat is transferred from the primary fluoride salt coolant to a secondary fluoride salt. The secondary fluoride salt in turn transfers its heat to a closed-loop Brayton electricity generation cycle. A conceptual drawing of a pebble bed FHR power plant proposed by UCB is shown in Fig. 1.

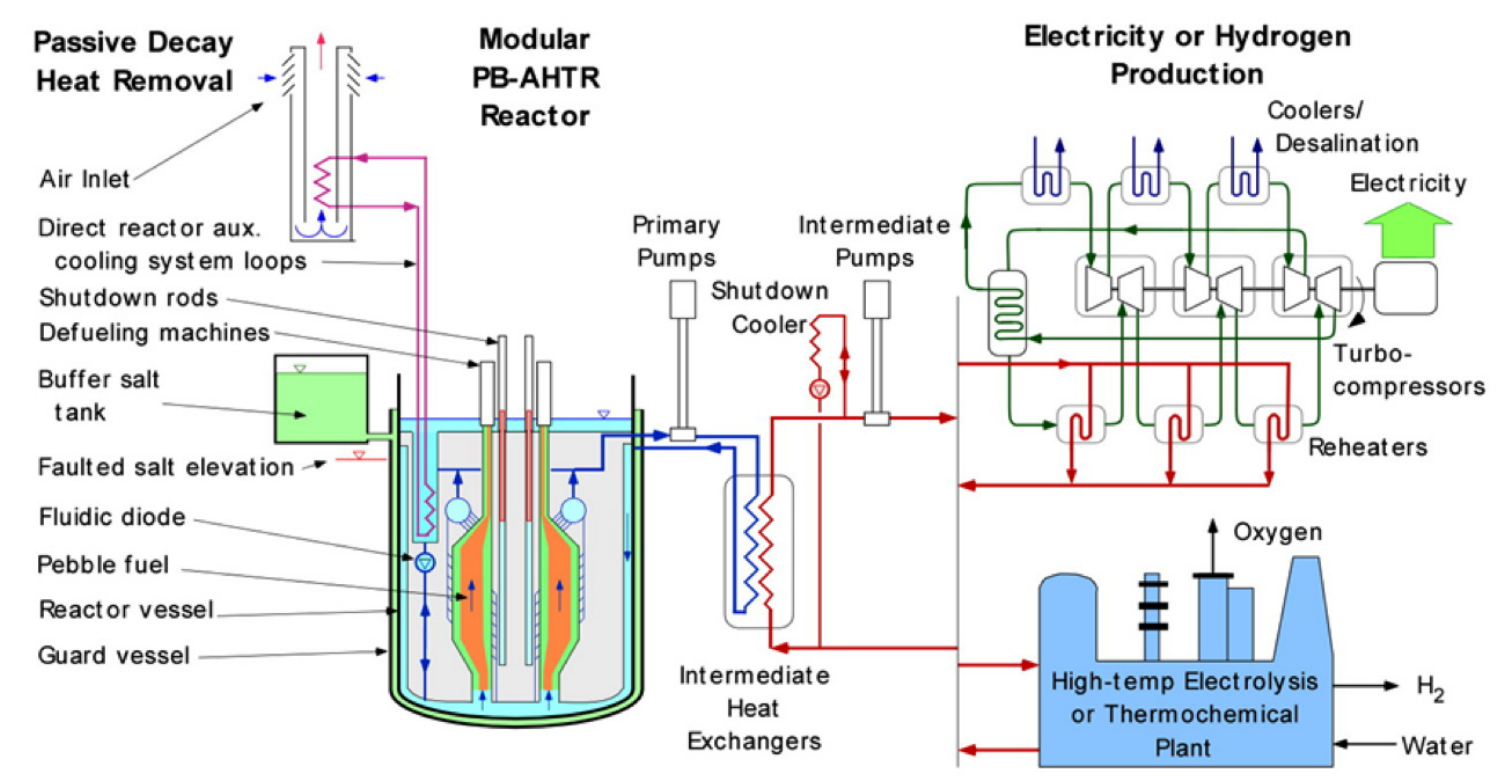

Fig. 1. Conceptual drawing of a pebble bed FHR and power generation cycle.

The defining elements of FHRs provide a very high degree of passive safety. Liquid fluoride salts have boiling points well above maximum reactor accident temperatures, allowing the reactor to remain at near atmospheric pressure and thereby avoiding the massive, expensive, high-pressure piping and vessel of water-cooled reactors. FHRs lack the potential to generate high pressure inside containment, inherently decreasing the containment structural requirements. The combination of liquid fluoride cooling and coated particle fuel provides several hundred degrees of temperature margin between worstcase accident scenario temperatures and fuel damage. Further, fluoride salts have high solubility for all fission products except the noble gases, providing an additional inherent safety barrier. Fluoride salts have high volumetric expansion with temperature, providing strong natural circulation cooling in the event of loss of forced convection cooling. Accident scenario decay heat removal is by passive, modular, natural circulation cooling.

The FHR's high outlet temperature increases the reactor thermal efficiency, lowering both the amounts of waste heat and spent fuel produced per unit energy and also enabling FHRs to serve as industrial process heat sources. The higher temperature cycle also greatly decreases cooling water requirements or alternatively facilitates economic dry cooling. The effective natural circulation heat transfer provided by liquid salts enables passive heat rejection to ambient air using a DRACS. The effective, passive heat removal allows large flexibility in reactor design. The reactor's low pressure, combined with the modular nature of its DRACS, enables FHRs to be developed at almost any power scale. Nuclear safety systems must be (with very high probability) continuously available over decades. Their reliability requirements build very large verification costs into nuclear safety system construction, operation, and maintenance. Because the safety characteristics of FHRs are inherent to their materials and physics, significantly fewer safety systems are required, helping to lower the overall reactor cost. 
FHRs share many properties with the dissolved fuel molten salt reactors that were developed and operated in the 1950s-60s. A considerable body of supporting information and understanding exists for this reactor type. Also, FHR coated particle fuel is similar to that being employed in high temperature helium-cooled reactors such as the Next Generation Nuclear Plant (NGNP). The NGNP project has established an extensive fuel development and test capability that is directly applicable to FHRs.

FHRs can be designed for on-line refueling much as existing Canada deuterium uranium reactors (CANDUs) or pebble bed reactors. Moving fuel is especially advantageous for an FHR, relative to gascooled reactors, because the FHR has much higher power density and consequent more rapid fuel burnup. In a fixed fuel FHR, maintaining a high power density along with an adequately long refueling interval is a principal fuel and core design challenge. Moving fuel FHRs also enable access to the thorium fuel cycle. In order to breed fissile material from fertile thorium, fuel would be repeatedly irradiated in the reactor periphery and moved ex-core between irradiation cycles to allow the generated ${ }^{233} \mathrm{~Pa}$ to decay into fissile ${ }^{233} \mathrm{U}$. No other proposed reactor class is as well suited for breeding ${ }^{233} \mathrm{U}$ within mechanically robust, chemically refractory, radiologically self-guarding fuel.

FHRs combine many technologies of varying levels of technological maturity into a system. Since the fuel, coolant, primary structural materials, safety basis, and power generation system differ substantially from current commercial reactors, substantial component and system level testing is necessary to provide confidence that the FHR concept will in practice perform according to its predictions and provide scientific and engineering data for the physics, chemistry, materials, and control systems necessary for designing and licensing FHRs.

\subsection{TECHNOLOGY BASE-OVERVIEW}

Investigation of molten salt reactors started in the late 1940s as part a U.S. program to develop a nuclear powered airplane. Experiments to establish the feasibility of molten salt fuels were begun in 1947. Molten fluoride salts were adopted in 1950 as the main line effort of the Aircraft Nuclear Propulsion (ANP) program. In carrying out this program, much information on the physical, chemical, and engineering characteristics of molten salt systems was obtained from studies of fluoride salt chemistry and materials compatibility, and from development of components, materials, fabrication methods, and reactor maintenance processes. In 1954, the Aircraft Reactor Experiment (ARE), a $2.5 \mathrm{MW}(\mathrm{t})$ molten salt reactor fueled with $\mathrm{UF}_{4}$ dissolved in a mixture of zirconium and sodium fluorides, moderated with beryllium oxide, was built and operated successfully for 9 days at steady-state outlet salt temperatures approaching $900^{\circ} \mathrm{C}$. No mechanical or chemical problems were encountered, and the reactor was found to be stable and self-regulating.

The Molten Salt Reactor (MSR) program was initiated in 1957, drawing upon the information developed in the ANP program as well as beginning new lines of investigation. By 1960 enough favorable experimental results were obtained to support authorization for design and construction of a 10-MW(t) Molten Salt Reactor Experiment (MSRE). Design of the MSRE started in the summer of 1960 , and construction started at the beginning of 1962. The reactor went critical in June 1965, and the MSRE initiated power operation in early 1966. The MSRE provided facilities for testing fuel salt, graphite, and Hastelloy $\mathrm{N}$ under reactor operating conditions. The basic reactor performance was outstanding and indicated that the desirable features of the molten salt concept could be embodied in a practical reactor that can be constructed, operated, and maintained safely and reliably. A photograph of the MSRE from above the reactor vessel is shown as Fig. 2. 


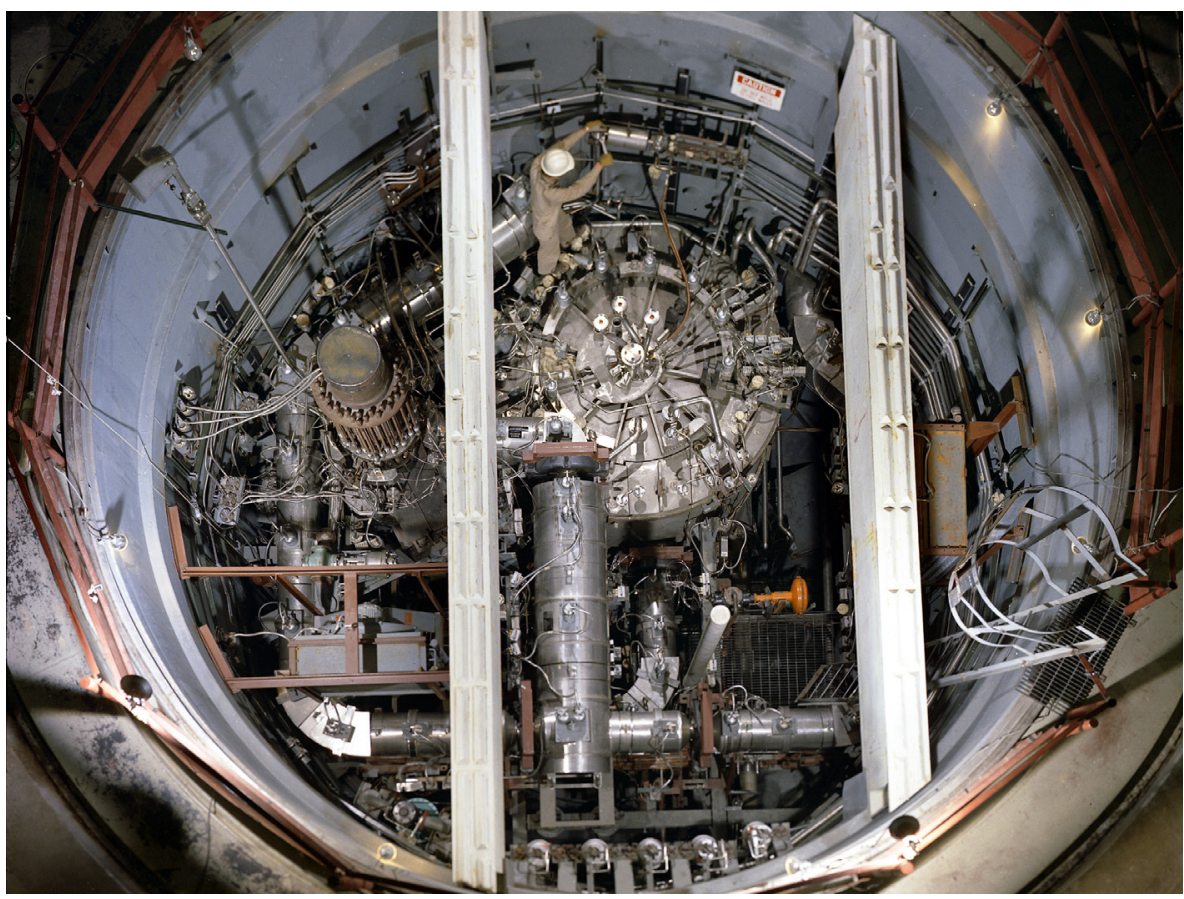

Fig. 2. The MSRE as seen from above.

The MSRE experience was of major importance to the molten salt concept. Until the MSRE began to operate well, few people besides those actively involved in the development program considered molten salt reactors to be really practical. The major reason was that operation and maintenance of a system containing a highly radioactive fluid fuel that melted at over $425^{\circ} \mathrm{C}$ seemed extremely difficult. In 1966, however, the MSRE began to provide evidence to offset that view. When power operation began, the usual start-up problems were encountered, but sustained power operation provided a remarkable demonstration of operability. Starting in late 1966, an uninterrupted one-month run was made, then a three-month run, and finally a six-month run. Next, using a small fluoride volatility plant connected to the reactor, the original partially enriched ${ }^{235} \mathrm{U}$ fuel was removed from the salt and was replaced by ${ }^{233} \mathrm{U}$ that had been made in a production reactor. The MSRE then operated a final year on the ${ }^{233} \mathrm{U}$, which made it the first reactor to ever have been operated on this fuel, and for a period plutonium was used as the makeup fuel. When shut down, the MSRE had circulated fuel salt at around $650^{\circ} \mathrm{C}$ for a total of 2.5 years. Perhaps the most important result from the MSRE was the conclusion that it was quite a practical reactor.

In 1972 ORNL proposed a major development program that would culminate in the construction and operation of a demonstration reactor called the Molten Salt Breeder Experiment (MSBE). In January 1973, ORNL was directed to terminate MSR development work. The program was reinstated a year later, and in 1974 ORNL submitted a more elaborate proposal calling for about \$720 million to be spent over an 11-year period. This last proposal was also rejected, and in 1976 ORNL was again ordered to shut down the MSR program for budgetary reasons.

In 2003 C. W. Forsberg, P. Pickard, and P. F. Peterson proposed combining the liquid fluoride salt coolant of the MSR with the coated particle fuel of gas reactors and the pool configuration of sodium fast reactors, and the FHR reactor concept was born [1]. An FHR class reactor was subsequently investigated and designated the Advanced High Temperature Reactor (AHTR). This reactor used coated particle fuel compacts embedded into prismatic graphite blocks and was designed at $1200 \mathrm{MW}(\mathrm{e})$ scale. 


\subsection{PRIMARY SOURCES OF INFORMATION}

The FHR test scale (FHR-TS) system description presented in this report is an amalgam of the UCB spring 2009 design report on the FHR-16 [2], an earlier design study for a 410-MW(e) pebble bed AHTR [3], the prior molten salt reactor program design documents, and the earlier ORNL prismatic fuel AHTR design [4] along with engineering judgment as to how an integrated system would need to be configured. The FHR-TS design remains incomplete and thus continues to evolve as more design details become available

A myriad of information is available from the prior MSR reactor program designs-ARE, MSRE, MSBE, and MSBR. A synopsis of the key prior molten salt reactor program documents used in preparation of this report follows.

\section{ORNL-TM-1855, “Components and Systems Development for Molten Salt Reactors”}

ORNL-TM-1855 had similar objectives, albeit on a molten salt fueled instead of a molten salt-cooled reactor, to the present effort and as such provided significant guidance on the content and structure of the analysis [5]. While AHTRs have significant technical differences from molten salt breeder reactors, the content and structure of this document are closely aligned to the prior analysis.

ORNL-TM-1855 is a components and systems status evaluation and gap identification report, similar to what is intended in this document. Evaluations in TM-1855 draw upon the engineering know-how and operating experience of molten salt reactor technology from earlier programs and initiatives including the MSRE. The immediate objective of the report was to describe the status of development of components and systems for molten salt reactors, and to present a rudimentary development program for the MSBE.

At the time TM-1855 was prepared, no structural design study had been made for the MSBE. The development program was based on a study of the problems of the available MSBR reference design with the assumption that the MSBE would be a "scaled-down" version of the modular MSBR. At the time, the MSBR was being planned to generate about 1,000 MW(e), and the MSBE was projected to have [less than] 200-MW(t) capacity. All components and systems for the MSBE were to be of a design that could be scaled up to the higher power level of the MSBR with major operating parameters kept the same.

These conditions have high resemblance to the present status of the FHR concept. The intention of this report to present the current state-of-the-art of the technology based on the past operating experience and more recent technological advancements. In a similar fashion to the MSBE, FHR-TS is considered an important intermediate step in reaching the ultimate goal of a commercial-scale AHTR.

\section{ORNL-TM-728, “MSRE Design and Operations Report, Part I-Description of Reactor Design”}

ORNL-TM-728 [6] provides extensive information on entire aspects of the design and operation of the MSRE. Even though this report was not written specifically for functional requirements of components but rather as a design document, the document includes significant information on the technology that was available at the time. ORNL-TM-728 also provides insight into the challenges that were faced during the development of particular systems, structures, and components.

\section{ORNL-TM-1993, "Experience with High-Temperature Centrifugal Pumps in Nuclear Reactors and Their Application to Molten-Salt Thermal Breeder Reactors"}

ONRL-TM-1993 [7] provides a compilation of design features, development problems, and operating experience for liquid-metal and molten salt circulating pumps used in various nuclear reactors and test 
facilities. The primary goal of the document is to search out the problem areas and to select suitable combinations of features for the circulating pumps required by each of the three molten salt systems-i.e., the fuel, the blanket and the coolant—in the proposed molten salt thermal breeder reactor. The pumps are divided into two major configurations: the "short-shaft pump" and the "long-shaft pump."

The document was then written to identify potential problem areas during the design and manufacturing stages of high temperature pumps. These challenges partly address current technological gaps, and development and testing requirements for the pumps that are expected to be used in the FHR-TS.

\section{ORNL-TM-2643, "Conceptual System Design Description of the Salt Pump Test Stand for the Molten Salt Breeder Experiment"}

ONRL-TM-2643 [8] presents the conceptual design of the salt pump test stand, including the description, function, and design requirements for components and subsystems. It also discusses the principles of operation of the test stand.

This report is particularly useful in identifying the testing requirements that were considered essential to be addressed by the test stand, thereby providing insight into the testing requirements for the pump test facility for the AHTR.

\section{ORNL-4812, "The Development Status of Molten-Salt Breeder Reactors"}

ORNL-4812 [9] is a status report on the MSBR program. Chapter 8 of this report is dedicated to reactor components and systems, including pumps, heat exchangers, valves, and control rods and drives. For each major component and system, the report gives a brief description followed by the operating experience. The report then tries to identify the status of the technology and the technological gaps to achieve a final product that will meet all the functional and operational requirements.

\section{ORNL-5018, "Program Plan for Development of Molten-Salt Breeder Reactors"}

ORNL-5018 [10] is a full program plan and technology status report on the MSBR program. Chapter 10 of the report discusses the roadmap for reactor technology development. The report describes the state-of-the-art of the technology as of 1975 by documenting the operating experience and development status of MSBR components and systems, such as pumps, valves, and steam systems. The report presents development plans by ten identified task groups, each focusing on a particular component or system.

\subsection{REPORT ORGANIZATION AND SCOPE}

This report first provides an overview of the current FHR-TS baseline design. The report coverage focuses on describing the component and system functioning as opposed to their materials or structure. Next the report provides an assessment of the technical maturity of the components and structures including both recent technological advances and the prior technology demonstrations within the MSR development program. Finally, the report presents an evaluation of the remaining development and testing tasks necessary before final design of an FHR-TS can take place. The report is organized to provide a bottom-up view of the component and system testing requirements, and component test facility recommendations are only those that arise from the testing requirements. 


\section{DESCRIPTION OF THE FHR TEST SCALE SYSTEM}

\subsection{SUMMARY}

\subsubsection{Introduction}

While UCB has developed baseline design information for much of the FHR-TS heat transport system, a complete design for an FHR-TS does not yet exist. This report describes the components and systems comprising one possible design variant for a pebble bed fluoride salt-cooled test reactor. The design information presented in this report is based upon a combination of the design and operational information of the MSRE, the UCB FHR-16 design report, the earlier prismatic fuel AHTR design, and a general understanding of the technological status and operating principles of an FHR. The system operational parameters presented are illustrative in nature, will almost certainly change as the system design progresses, and consequently should not be relied upon for making definitive design decisions.

The MSRE had a maximum operating thermal power level of 7.4 MW and a core exit temperature of $650^{\circ} \mathrm{C}$. Based upon very preliminary thermal performance modeling, it is estimated that sufficient scale similarity exists between an FHR test reactor and a larger commercial unit such that essential thermal performance data can be obtained with a $<20 \mathrm{MW}(\mathrm{t})$ test reactor provided that the coolant entrance and exit temperatures as well as core peak power density match between the test and commercial units.

The FHR-TS baseline design calls for an annular pebble bed core. The FHR-TS baseline primary coolant is a lithium fluoride-beryllium fluoride $\left(66-34 \mathrm{~mole} \%, 99.99+\%{ }^{7} \mathrm{Li}\right)$ salt commonly referred to as flibe. The upper limit for the core outlet temperature in the FHR-TS is determined primarily by the structural materials of the primary piping and heat exchangers as all other reactor components have significantly higher temperature tolerance. Hastelloy- $\mathrm{N}$ is the highest temperature rated, currently available alloy for liquid salt reactor pressure vessel service. Use of Hastelloy-N as the coolant pressure boundary limits the reactor temperature to $704^{\circ} \mathrm{C}$ as Hastelloy-N softens significantly at higher temperatures.

Higher temperature tolerant alloys, however, are currently under consideration as part of the high temperature gas-cooled reactor (HTGR) development program. Notably, alloys $800 \mathrm{H}$ and 617 have significant residual strength to over $850^{\circ} \mathrm{C}$. While neither of these alloys is chemically compatible with fluoride salts at high temperatures, nickel is highly compatible with the salt, and if a nickel-based clad high temperature material system can be proven, the FHR-TS outlet temperature would increase to the limits of the new alloy. Because the lower pressure of an FHR as compared to an HTGR results in lower stress on the primary pressure boundary materials, the FHR-TS may be able to use HTGR developed alloys to significantly higher temperatures than they would be useful for at a gas-cooled reactor with the same core outlet temperature.

A primary purpose for the FHR-TS is to provide representative nuclear, thermal, hydraulic, and control phenomena so as to provide confidence, through benchmarking, in the reactor modeling tools used to design larger-scale systems. The key fuel and neutronic scaling requirement is to achieve a similar power and neutron flux density within the test reactor core independent of volume. Neutronic phenomena thus influence the minimum core size primarily through the necessity to remain critical while only employing low-enrichment fuel. In general, technological challenges (corrosion, instrumentation endurance, material strength, etc.) increase with increasing temperatures. Hence an FHR experimental reactor program has a strong motivation to operate at full commercial reactor temperatures. Also, a reasonably high operating temperature is required for testing the reactor's value and capability to drive a closed gas Brayton power cycle. Additionally, FHRs have an inherent minimum operating temperature 
range arising from the freezing point (and relatively high viscosity of the salts slightly above their melting points) of the selected primary coolant salt $\left(\sim 460^{\circ} \mathrm{C}\right)$.

\subsubsection{System Diagram}

The FHR-TS energy flow will mimic that of a larger power reactor. However, some system changes are required because of the smaller size. The principal concessions to the reduced scale are that the fuel will require higher enrichment (although remaining below the $20 \%$ threshold for low enrichment) and that only a single heat transport loop will be employed. An overall primary heat transfer system flow diagram is shown as Fig. 3.

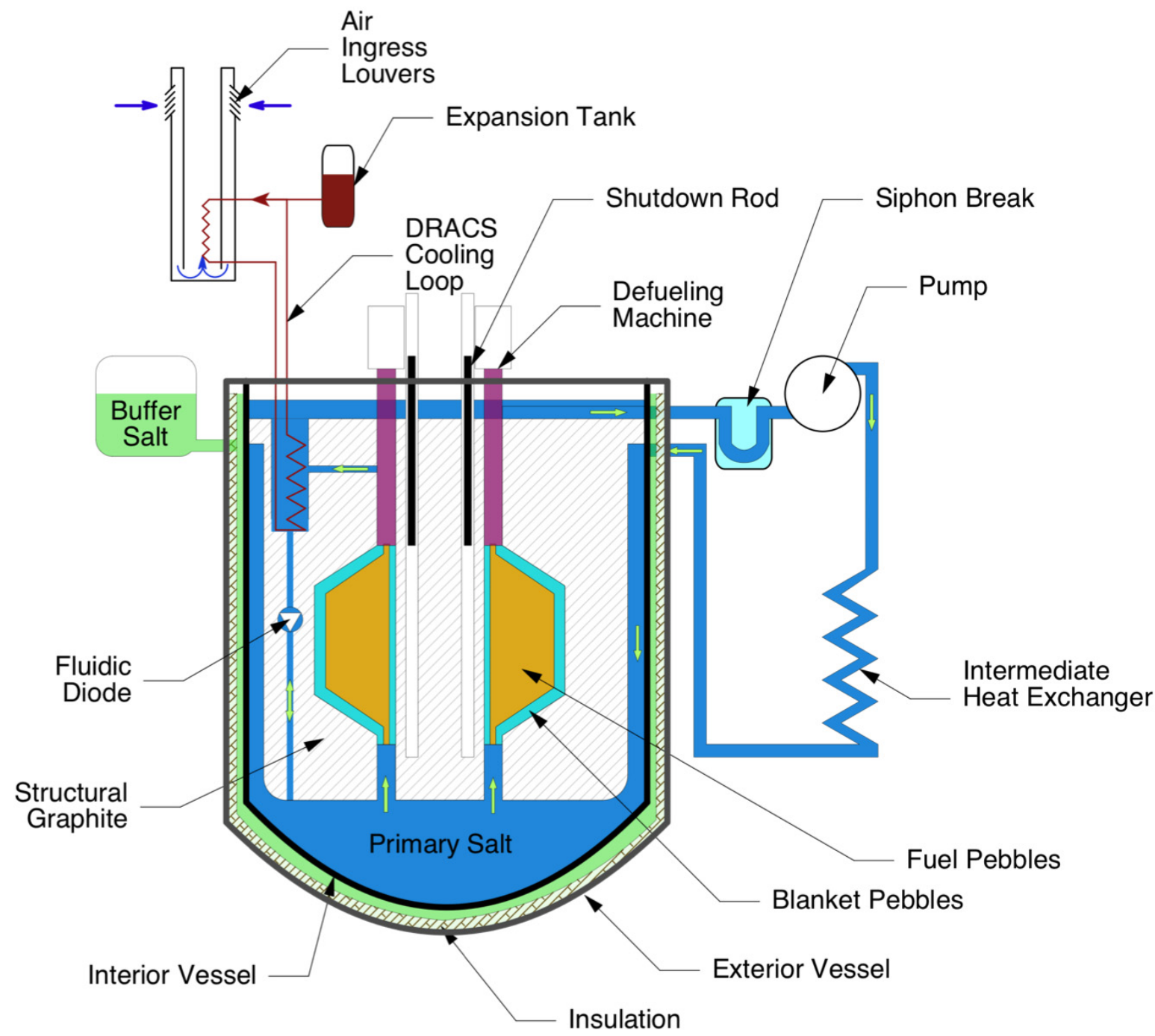

Fig. 3. Overall FHR-TS primary heat transfer flow.

While UCB has proposed a baseline configuration, several key system layout issues remain unresolved at the present time. For example, the intermediate heat exchanger may be located within the reactor vessel to both mitigate the safety consequences for primary loop piping breaks along with minimizing the required amount of isotopically selected primary salt. Alternatively, the intermediate heat 
exchanger may be located ex-vessel and sufficient salt volume located above the core to flood the surrounding dry-well such that in the event of a large break in the vessel or primary piping, the core remains covered with salt. Further, whether insulation can be placed between the interior and exterior reactor vessels to lower the exterior vessel temperature will only be ascertained as additional system materials development is performed. Additionally, whether the power generation cycle will employ a nitrogen-helium mixture or supercritical carbon dioxide has not yet been determined. A process flow block diagram for the FHR-TS is shown as Fig. 4, and a comparison of FHR-TS and the MSRE general design parameters is included as Table 1. The green color in Fig. 4 represents the normal operation systems. The black color in Fig. 4 represents the maintenance or shutdown systems, and the red color represents the loss of forced cooling accident condition systems.

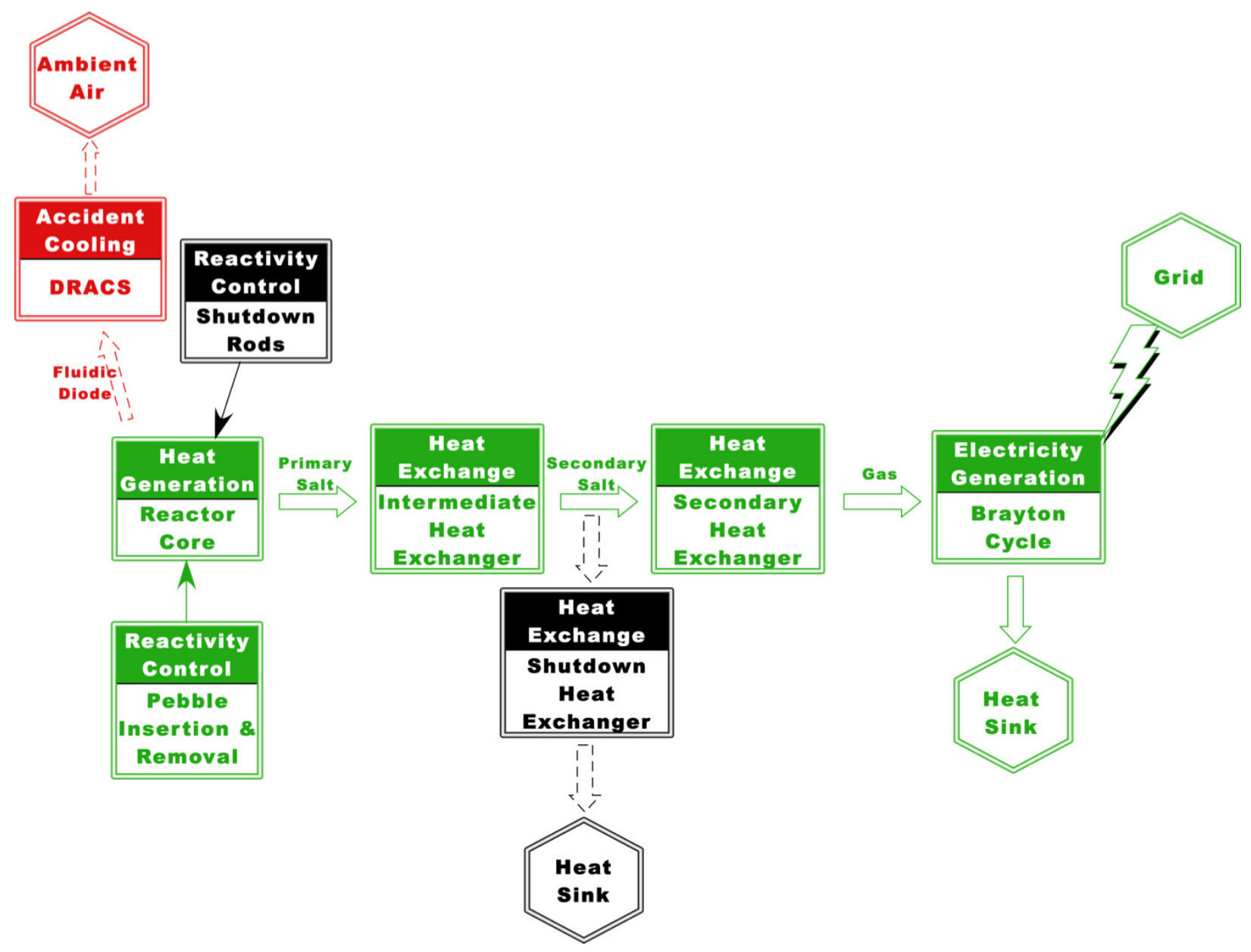

Fig. 4. FHR-TS process flow block diagram. 
Table 1. FHR-TS general design parameters as compared to the MSRE

\begin{tabular}{|c|c|c|c|}
\hline & FHR-TS & MSRE & Units \\
\hline \multicolumn{4}{|l|}{ General Plant Data } \\
\hline Reactor thermal output & 16 & 7.4 & $\mathrm{MW}(\mathrm{t})$ \\
\hline Power plant output, gross & 7.3 & $\mathrm{~N} / \mathrm{A}$ & MW(e) \\
\hline Power plant output, net & 7.0 & $\mathrm{~N} / \mathrm{A}$ & MW(e) \\
\hline Power plant efficiency, net & 43.8 & $\mathrm{~N} / \mathrm{A}$ & $\%$ \\
\hline \multicolumn{4}{|l|}{ Reactor Core } \\
\hline Active core height & 1.00 & & $\mathrm{~m}$ \\
\hline Average core power density (driver region) & 20 & & $\mathrm{MW} / \mathrm{m}^{3}$ \\
\hline Outer diameter of pebble & 30 & $\mathrm{~N} / \mathrm{A}$ & $\mathrm{mm}$ \\
\hline Total number of pebbles in core (all regions) & 97,000 & $\mathrm{~N} / \mathrm{A}$ & \\
\hline Number of driver pebbles & 42,000 & $\mathrm{~N} / \mathrm{A}$ & \\
\hline Number of blanket pebbles & 55,000 & $\mathrm{~N} / \mathrm{A}$ & \\
\hline Number of driver pebbles in active region & 34,000 & $\mathrm{~N} / \mathrm{A}$ & \\
\hline \multicolumn{4}{|l|}{ Primary Coolant System } \\
\hline Primary coolant flow rate (MSRE estimated) & 65 & 140 & $\mathrm{~kg} / \mathrm{s}$ \\
\hline Reactor operating pressure & Atmospheric & & $\mathrm{MPa}$ \\
\hline Core coolant inlet temperature & 600 & 632 & ${ }^{\circ} \mathrm{C}$ \\
\hline Core coolant outlet temperature & 704 & 654 & ${ }^{\circ} \mathrm{C}$ \\
\hline \multicolumn{4}{|l|}{ Intermediate Coolant System, if applicable } \\
\hline Intermediate coolant flow rate & 55 & 105 & $\mathrm{~kg} / \mathrm{s}$ \\
\hline Intermediate coolant operating pressure & Atmospheric & & $\mathrm{MPa}$ \\
\hline Intermediate coolant minimum temperature & 548 & 546 & ${ }^{\circ} \mathrm{C}$ \\
\hline Intermediate coolant maximum temperature & 690 & 579 & ${ }^{\circ} \mathrm{C}$ \\
\hline \multicolumn{4}{|l|}{ Ultimate Heat Rejection System } \\
\hline Working medium & $\begin{array}{l}\text { High-pressure } \\
\text { nitrogen-helium or } \\
\text { supercritical } \mathrm{CO}_{2}\end{array}$ & $\begin{array}{l}\text { Atmospheric } \\
\text { pressure air }\end{array}$ & \\
\hline Working medium flow rate (nominal conditions) & 5.5 & & $\mathrm{~kg} / \mathrm{s}$ \\
\hline Working medium pressure & 10 & & MPa \\
\hline Working medium upper temperature & 675 & & ${ }^{\circ} \mathrm{C}$ \\
\hline Working medium lower temperature & 495 & Ambient & ${ }^{\circ} \mathrm{C}$ \\
\hline \multicolumn{4}{|l|}{ Reactor Vessel } \\
\hline Outer diameter of cylindrical shell & 1.35 & & $\mathrm{~m}$ \\
\hline Design pressure & 0.5 & & $\mathrm{MPa}$ \\
\hline Design temperature & 800 & & ${ }^{\circ} \mathrm{C}$ \\
\hline \multicolumn{4}{|l|}{ Guard Vessel } \\
\hline Design pressure & 0.2 & & $\mathrm{MPa}$ \\
\hline Design temperature & 600 & & ${ }^{\circ} \mathrm{C}$ \\
\hline
\end{tabular}




\subsection{REACTOR CORE SYSTEMS}

The FHR-TS design features an annular pebble bed reactor core. A photograph of the UCB FHR-16 core model, which shows a vertical, $30^{\circ}$ thick cross section of the annular core, is shown as Fig. 5 . The comparatively simple heat transfer, flow, and mechanical motion characteristics of single-phase, welllubricated liquid systems such as the FHR primary coolant and fuel facilitate the use of computational models and simulant materials to predict real system thermal and hydraulic phenomena. Initial surrogate material simulation of the lubricating nature of the fluoride salt for the pebble surface, coolant, and graphite reflector walls indicates that pebbles will progress in an orderly manner up through the core, remaining at the radial location at which they were inserted as they traverse the core height while avoiding the pebble grinding and clumping that has been problematic with helium-lubricated pebble beds.

The UCB system model uses dry plastic spheres that proceed down through the core, whereas the actual system will have liquid salt lubrication, upward fuel pebble motion, and upward salt flow. The UCB baseline FHR-16 design specifies a $16 \mathrm{MW}(\mathrm{t})$ core power and a core power density of $20 \mathrm{MW} / \mathrm{m}^{3}$. As can be seen in the figure, the core design calls for a diverging entrance region and converging exit region. The core is designed to be critical only at the expanded diameter, greatly reducing the flux to the entrance and exit region materials. To further reduce the neutron flux on the surrounding graphite blocks, the fuel can be radially zoned such that either unfueled graphite or breeder pebbles are located adjacent to the fixed graphite. The details of the tribology of fluoride salt and carbon surfaces are an additional research and development (R\&D) element for pebble fuel cores.

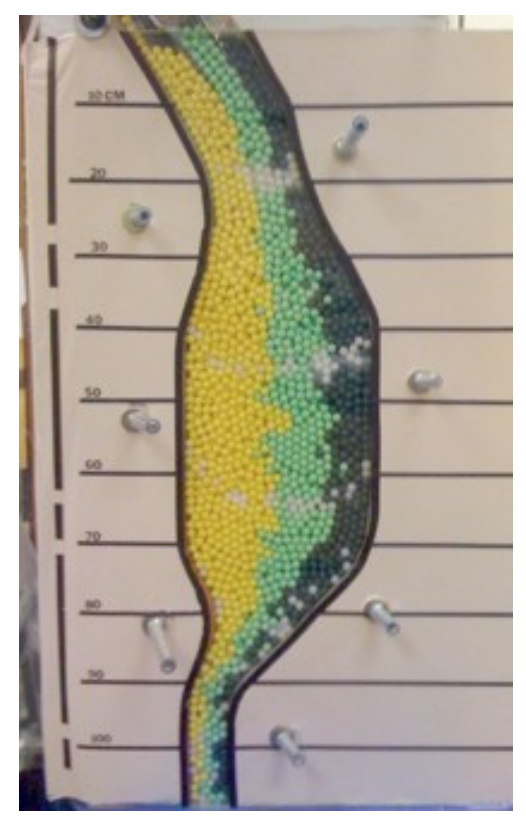

Fig. 5. Photograph of UCB FHR-TS core model.

\subsubsection{Fuel Elements}

The fuel pebbles of the FHR-TS are a primary element of the reactor's energy conversion system. The nuclear and fabrication aspects of the fuel pebbles are outside the scope of component testing. The current FHR-TS design calls for the fuel pebbles to have a smaller diameter than the pebble bed gascooled reactor to provide larger contact surfaces between the coolant and fuel (roughly $3 \mathrm{vs} 6 \mathrm{~cm}$ in diameter). A fuel pebble would consist of a low-density graphite center (to provide slightly positive buoyancy) surrounded by a fueled annulus containing a bed of as high as possible density coated particle 
fuel. The fueled annulus would be covered by a thin, protective, high-density graphite outer surface. An illustration of a fuel pebble and the layering of a coated fuel particle is shown in Fig. 6.

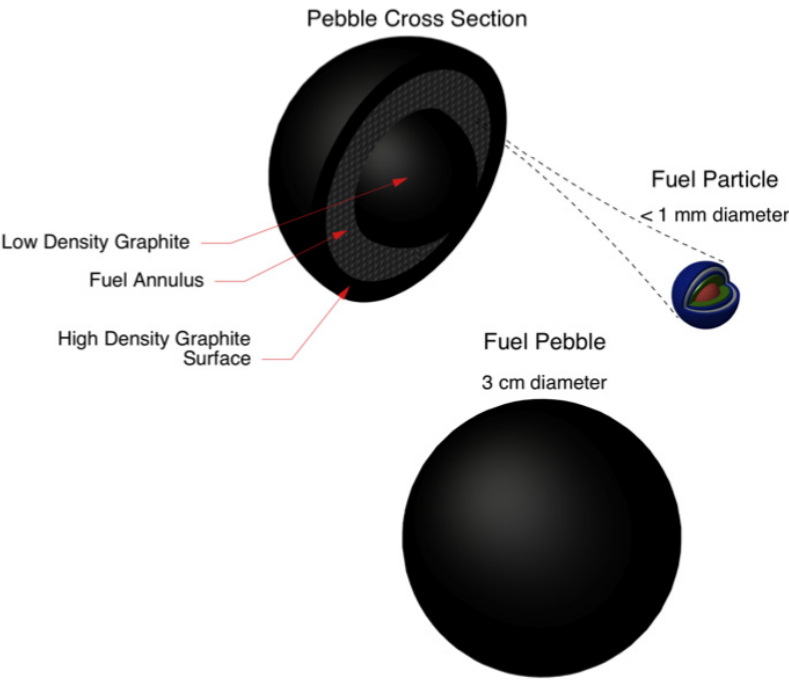

(a)

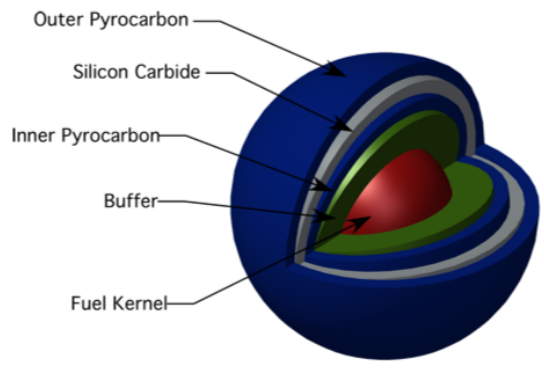

(b)

Fig. 6. FHR (a) fuel pebble, fuel pebble cross section, and coated particle; (b) enlarged view of a coated particle.

The flow resistance of the bed of pebbles; the heat transfer from individual pebbles; the friction, lubrication, and wear of the pebbles against each other and the other primary circuit elements; as well as any potential loss of buoyancy due to coolant density decrease at higher temperature are all phenomena requiring evaluation.

\subsubsection{Reactivity Control and Shutdown System}

The FHR has intrinsic, negative coolant and fuel temperature reactivity feedback. The reactivity control and shutdown systems control the reactivity in the core by adjusting the amount of fuel within the core for slow transients and provides a means for rapid reactor control and shutdown by inserting control and shutdown rods either into the central graphite island and/or the outer radial reflector. The FHR-TS is intended to operate without control rods or embedded poisons. The primary operational reactivity control method is to insert and/or remove pebbles from the core. Flow control is also possible due to the combination of strong negative temperature reactivity feedback, the excellent cooling properties of the salt, and the robust nature of the fuel. The achievable power ramp rate is thus determined by the combination of the ability of the fuel handling system to add and/or remove pebbles and the primary pump maximum flow rate. Therefore, the fuel handling system rate capability needs to factor the desired control response into its design.

Primary components of the reactor shutdown system include shutdown links or rods, interlinking components, guide tubes, and a drive mechanism. The mechanical, temperature, and flux requirements for the shutdown elements are nearly identical between salt- and gas-cooled high temperature reactors. Consequently, extensive use is anticipated of the existing HTGR control element designs [11]. An illustration of a typical segment of shutdown absorber rod control element is shown in Fig. 7. The specific design and material selection for shutdown absorbers have not yet been finalized. The guide tubes for the shutdown elements may either be salt filled tubes hydraulically interconnected to the primary coolant or cover gas (argon) filled and cooled thimbles. The low pressure of the FHR combined 
with the elements only being employed for shutdown reduces the shutdown rod thimble strength and cooling requirements, providing greater design flexibility. Boron carbide $\left(\mathrm{B}_{4} \mathrm{C}\right)$ is a leading candidate material for the shutdown elements. Leading candidate materials for the shutdown rod guide tubes are silicon carbide; silicon carbide composites; carbon-carbon composites; and nickel-based alloy-clad, high temperature tolerant, low neutron absorption metals such as $\mathrm{Nb}-1 \mathrm{Zr}$.

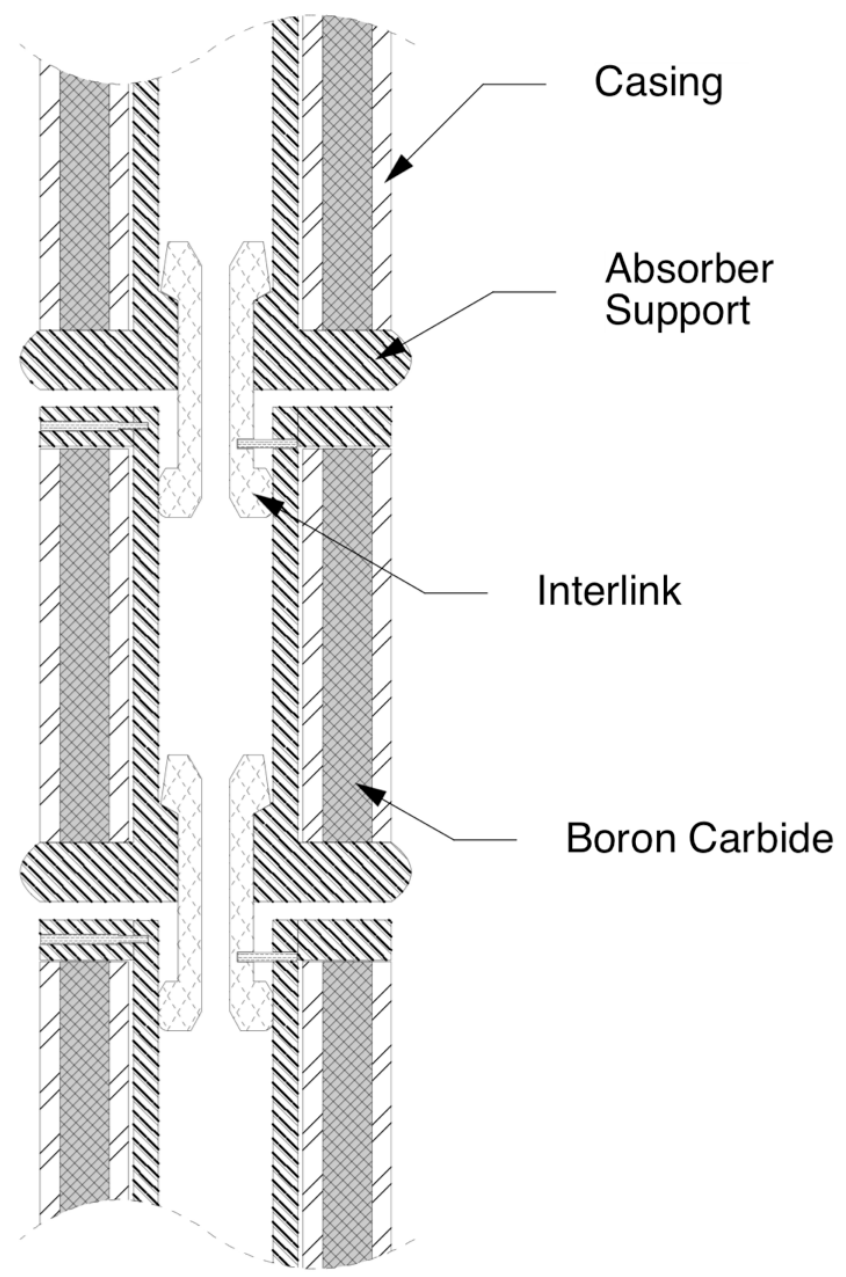

Fig. 7. Cross section of a high temperature reactor shutdown absorber stack.

A potential secondary shutdown method is to inject poisoned salt (e.g., potassium fluoroborate) into guide tubes located near the core within the reflector graphite similar to the shutdown rod guide tubes. The poisoned salt injection system would be similar to the lithium injection modules proposed by Kambe and Uotani [12]. In this type of system, as illustrated in Fig. 8, pressurized gas forces poisoned salt into the guide tube region of the shutdown module if the reactor temperature exceeds the freeze plug melt point. Restarting the reactor following the activation of the secondary shutdown modules would require removing and resetting the modules. 


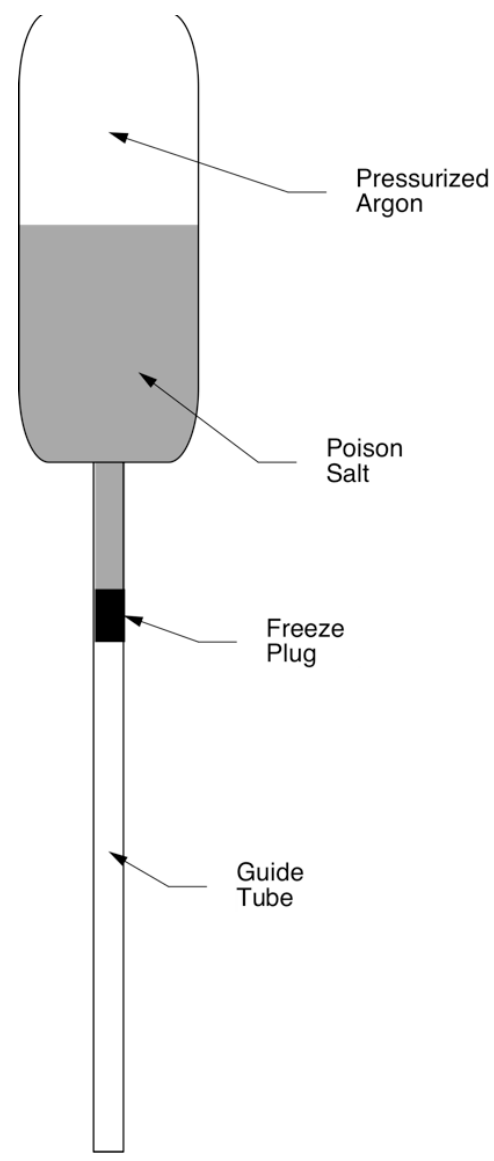

Fig. 8. Poisoned salt secondary shutdown system.

The shutdown system must have both a normal shutdown capability and a reserve shutdown capability, to provide sufficient reliability. The optimal combination of shutdown methods, including the specific method used to provide reserve shutdown, has yet to be determined. Earlier MSRs used shutdown rods inserted into a closed thimble, with the rod mechanism cooled by a gas stream (see Fig. 18 in Sect. 3.2.2). Alternately, rods could be guided directly in the salt environment. In the latter case, shutdown rods may be designed to be neutrally buoyant in flibe at $615^{\circ} \mathrm{C}$, so that they will passively insert upon loss of heat sink, providing a reserve shutdown capability in addition to the forced insertion of the shutdown rods.

\subsubsection{Fuel Handling and Storage System}

The Fuel Handling and Storage System (FHSS) performs the following fuel manipulations required during the life cycle of the FHR-TS.

- Initial fuel loading and start-up of the core.

- Replenishing the core inventory to compensate for burnup. This includes discharged fuel pebbles from the core, assessing the identity and burnup level of individual pebbles, and either returning the discharged pebbles into the bottom of the core or replacing spent pebbles with appropriate fresh pebbles.

- Under direction of the plant control and safety systems, maintaining the core fuel inventory appropriately to match the desired core reactivity. 
- Discharging the spent pebble fuel to spent fuel storage tanks, or possibly to a pebble reconditioning facility.

- Maintaining the radial and possibly axial profile of pebbles in the core, including fuel pebbles, graphite reflector pebbles, and possibly blanket pebbles containing fertile material such as thorium.

An early concept for the pebble fuel handling system for an FHR pebble bed commercial-scale reactor is shown in Fig. 9. This injection and defueling method has been demonstrated using water experiments scaled to match the friction, form drag, and buoyancy forces acting on the pebbles [13].

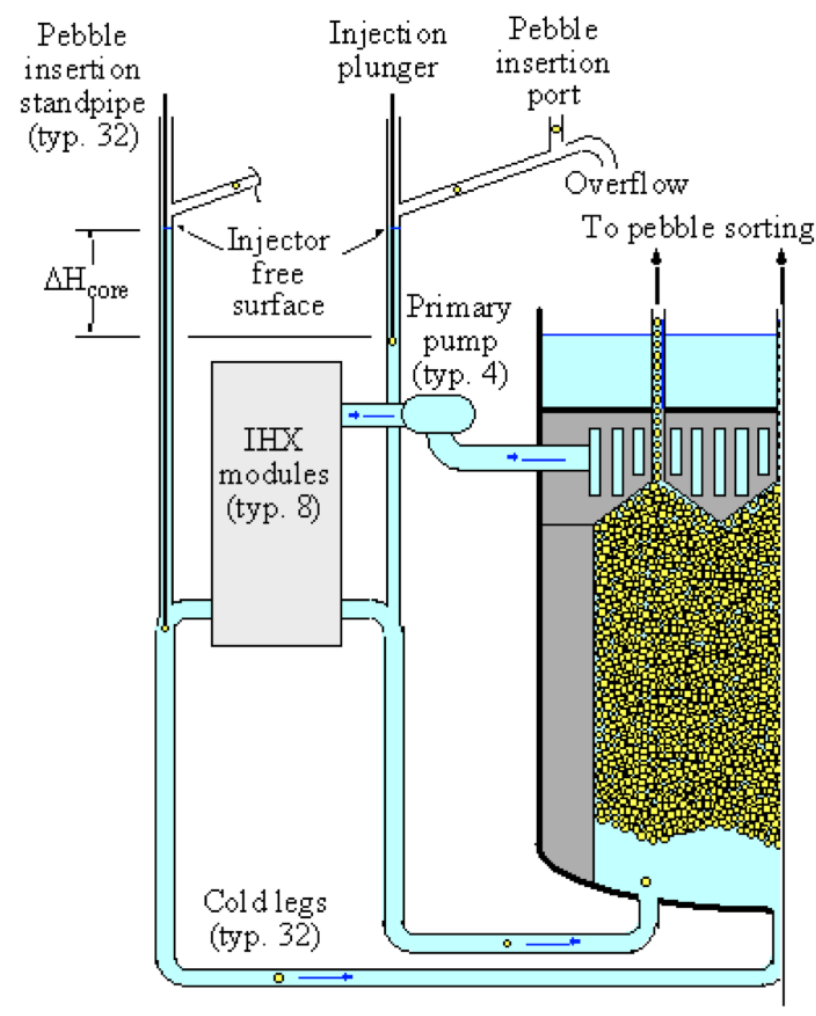

Fig. 9. A conceptual drawing of fuel pebble handling in an FHR.

(Courtesy of University of California-Berkeley)

The FHR-TS FHSS consists of a salt-filled portion and a gas-filled portion. The salt portion, interacting with the coolant circuit, is used to deliver and remove pebbles from the core. The gas portion is used to pneumatically transfer pebbles from (and possibly to) the reactor assembly. A key analysis, to be performed as the overall reactor design evolves, will determine the minimum decay time required before pebbles can transition from the salt environment into a gas-cooled environment.

As pebbles leave the core, they will be scanned by an identification system and sorted as to fuel, graphite, and possibly fertile content. Irradiated fuel or blanket pebbles will be tracked to estimate burnup; radiation measurement data will be used to match predicted burnup for the pebble. A determination of the physical condition of the pebble will also be made. These data will be used to decide whether the pebble is returned to the core or sent to storage, disposal, or a reconditioning facility. A single fuel pebble is expected to make a minimum of six passes through the core before it is transferred to the spent fuel storage system. 
The baseline design for the pebble storage system consists of multiple vertical, cylindrical, passively cooled storage tanks. Pebbles are introduced at the top and can be removed from the bottom of these tanks. A ladder structure in the center of the tank is designed to allow pebbles to drop into the tank with a large number of short falls, to prevent damage.

\subsection{HEAT TRANSPORT SYSTEM}

The FHR-TS heat transport system is an ensemble of two distinct but thermally coupled systems: the Primary Heat Transport System (PHTS) and the Intermediate Heat Transport System (IHTS). Under power operation the PHTS transports the heat generated by the reactor core to the IHTS through the Intermediate Heat Exchanger (IHX). The heat is ultimately transferred to the Power Conversion System (PCS).

The PHTS architecture is based on passively safe reactor design principles. Safety is assured primarily by virtue of the design, material properties, and by means of passive protection systems to provide adequate cooling and prevent overcooling at all times. High heat capacity of the fuel, graphite moderator, and coolant assures that the decay heat can be absorbed a substantial period of time without fuel damage.

The functional requirement of the PHTS is to provide and maintain adequate primary liquid salt flow rate to keep reactor temperature within the design limits to preclude damage to the reactor vessel, fuel, and reactor internals during normal operating and shutdown conditions. The configuration of the PHTS enables natural circulation flow to the IHX under shutdown and accident conditions, as well as natural circulation flow to the DRACS (see Sect. 2.4) heat exchangers. Decay heat can be removed by operation of the PCS, by natural circulation to a normal Shutdown Cooling System (SCS) heat exchanger in the intermediate loop, and by natural circulation flow to the DRACS. Because the combined capacity of the PCS, SCS, and DRACS systems substantially exceeds reactor decay heat generation, all are designed to control the heat rejection rate by limiting the rate of heat removal to the ultimate heat sink fluid (component cooling water for the SCS, and external ambient air for the DRACS).

Due to high melting temperature of salts, a design option is for the PHTS components to be housed in an insulated reactor cavity that acts as a furnace and equipped with electrical heating devices to maintain a sufficiently high cavity temperature to prevent the primary coolant from freezing. The intermediate loop piping, which extends outside the reactor cavity, would be heat traced and insulated and connected to a drain tank. Table 2 shows some selected PHTS operational parameters.

Table 2. Primary Heat Transport System baseline parameters

\begin{tabular}{ll}
\hline Primary Coolant & Liquid salt $\left({ }^{7} \mathrm{Li}_{2} \mathrm{BeF}_{4}\right)$ \\
Primary Vessel Cover Gas & Argon \\
Guard Vessel Cover Gas & Argon \\
Core Bulk Outlet Temperature & $704^{\circ} \mathrm{C}$ \\
Core $\Delta \mathrm{T}$ & $104^{\circ} \mathrm{C}$ \\
Primary Circuit Pressure & Near Atmospheric \\
\hline
\end{tabular}

\section{Liquid salt}

The FHR-TS is intended to use liquid fluoride salt as the primary, intermediate, and DRACS coolants. The primary coolant flows through the reactor core, through the hot legs into the primary coolant pumps, through the intermediate heat exchangers, and back to the reactor vessel through the cold 
legs and the downcomer. The primary salt coolant circuit also forms part of the fuel pebble transport system, which is described in Sect. 2.2.3.

The primary liquid salt should satisfy the following operational requirements:

1. Exhibit chemical stability at temperatures above $850^{\circ} \mathrm{C}$.

2. Maintain stability under intense radiation.

3. Melt at useful temperatures (i.e., below $525^{\circ} \mathrm{C}$ ) and not be substantially volatile.

4. Be compatible with high-temperature alloys and graphite.

Salt performance requirements were first established by Grimes [14] during the MSBR and MSRE programs, and still apply to the considerations for selecting liquid salt for the FHR-TS primary, intermediate, and DRACS coolants. Williams et al. [15] have recently updated Grimes' original assessment focusing on the specific requirements of an FHR.

From a neutronics standpoint, the primary coolant should exhibit sufficiently low parasitic neutron capture cross section and sufficiently high moderating capability to provide a negative void reactivity coefficient. Flibe has excellent material compatibility and very low corrosion rates with high nickel alloys provided that proper chemistry control practices are used. Flibe's activation products include tritium, which needs to be actively managed during reactor operation. The remaining activation products have short half-lives, which reduces radiation levels associated with the coolant [4]. The use of flibe as the primary salt does, however, require use of isotopically pure $(99.99+\%){ }^{7} \mathrm{Li}$. Further, the beryllium in flibe is a potentially fatal irritant and as such will have to be controlled throughout the FHR lifecycle.

The IHTS transfers heat generated by the reactor core from the PHTS to the PCS, providing sufficient heat removal rate to maintain reactor temperature within prescribed limits, and to prevent damage to the reactor vessel, fuel, and other reactor internals during normal operation, shutdown and under various upset conditions. The IHTS is thermally coupled to the PHTS through an Intermediate Heat Exchanger (IHX), and to the PCS through the secondary heat exchanger.

The intermediate coolant flows from the IHX, through the secondary coolant loop hot leg to an intermediate pump, through the secondary heat exchanger, and back into the IHX through the secondary coolant loop cold legs. The intermediate coolant transfers the heat from the PHTS to the PCS. The intermediate loop also includes a Shutdown Cooling System (SCS) heat exchanger. The intermediate loop is configured to operate with natural circulation when the intermediate pump is not operational, and a fluidic diode in the SCS inlet piping initiates natural circulation flow after the intermediate pump is stopped.

The FHR-TS uses liquid fluoride salt as the intermediate coolant, albeit a different salt than the primary coolant. A final decision has not yet been made, but a number of candidate salts exist for the intermediate coolant, with $\mathrm{KF}_{\mathrm{Z}} \mathrm{ZrF}_{4}, \mathrm{KF}_{-} \mathrm{KBF}_{4}$, and LiF-NaF-KF (flinak) being the lead candidate salts. The primary concern for the use of flinak in the intermediate heat transfer loop is in the potential for leaks within the IHX that dilute the expensive isotopically separated ${ }^{7} \mathrm{Li}$ of the primary coolant. Williams has recently published an assessment of candidate salts for an intermediate heat transfer loop [16].

The IHTS liquid salt inventory can be transferred to a temporary storage tank under certain conditions such as maintenance. The IHTS has electric heat tracing capable of maintaining the liquid salt in a liquid state, if required during normal plant operations as well as maintenance. 
The following subsections provide more detailed descriptions of heat transport system components of the FHR-TS.

\subsubsection{Reactor Vessel and Primary Piping}

The reactor vessel constitutes the primary boundary for the primary liquid salt coolant and its cover gas, and supports the reactor and reactor internal structures. The reactor vessel also provides a hermetically sealed containment boundary around the primary system fluids. It is initially planned that the reactor vessel will be designed for a service life of 60 years.

A salient feature of the FHR-TS design is that it avoids penetrations in the lower sections of the reactor vessel to eliminate a number of potential failure modes. The hot and cold legs are the points where the primary coolant leaves and returns to the reactor vessel boundary. Both the hot and cold legs leave the reactor vessel near the top and the hot leg includes a safety-grade suction break to preclude lowering the primary coolant level within the vessel. The hot and cold legs are relatively short to provide a compact configuration for the PHTS. Thermal expansion is accommodated by placing the IHXs on sliding bearing surfaces.

The FHR-TS does not have a completed reactor vessel design, and material technology developments will dominate the eventual reactor vessel configuration. The reactor vessel for the FHR-TS is likely to be a layered structure. The baseline design reactor vessel layer structure is shown in Fig. 10. The interior vessel functions essentially as an open top tub to provide high temperature containment of the primary coolant salt. Carbon-carbon composite is currently the leading candidate material. The next material layer will most likely be a poisoned buffer fluoride salt with a lower melting temperature than the primary salt. The buffer salt layer provides (1) hydraulic support to the interior vessel, (2) chemically similar environments on both sides of the interior vessel, and (3) a strong negative reactivity insertion into the reactor in the event of interior vessel failure. Surrounding the buffer salt will be a fluoride saltcompatible thermal insulation layer. The thermal insulation layer function is to lower the temperature requirements of the exterior reactor pressure vessel. The layered structure would enable the FHR-TS vessel to be fabricated from well-known reactor ASME Class 1 steel. Alternatively, the FHR-TS vessel could be fabricated from a higher temperature alloy such as Alloy $800 \mathrm{H}$.

The FHR-TS design's double vessel provides two separate barriers to loss of coolant and radioactive material release from the core. A buffer salt inventory tank located above the inner and guard vessels keeps the buffer salt at a slightly higher pressure than the maximum anticipated pressure within the reactor vessel, and provides makeup if the interior reactor vessel becomes faulted. The buffer salt will be a poisoned lower melting point fluoride salt such as $\mathrm{KF}-\mathrm{ZrF}_{4}$. The lower melting point of the exterior salt enables the guard vessel to be maintained at a lower temperature during normal operation. The net volume of the guard vessel will be sufficiently small, and the buffer tank inventory sufficiently large, so that following an interior reactor vessel failure the buffer salt inventory will maintain the primary liquid salt level in the reactor vessel at an elevation that will allow natural circulation for cooling of the reactor core. 


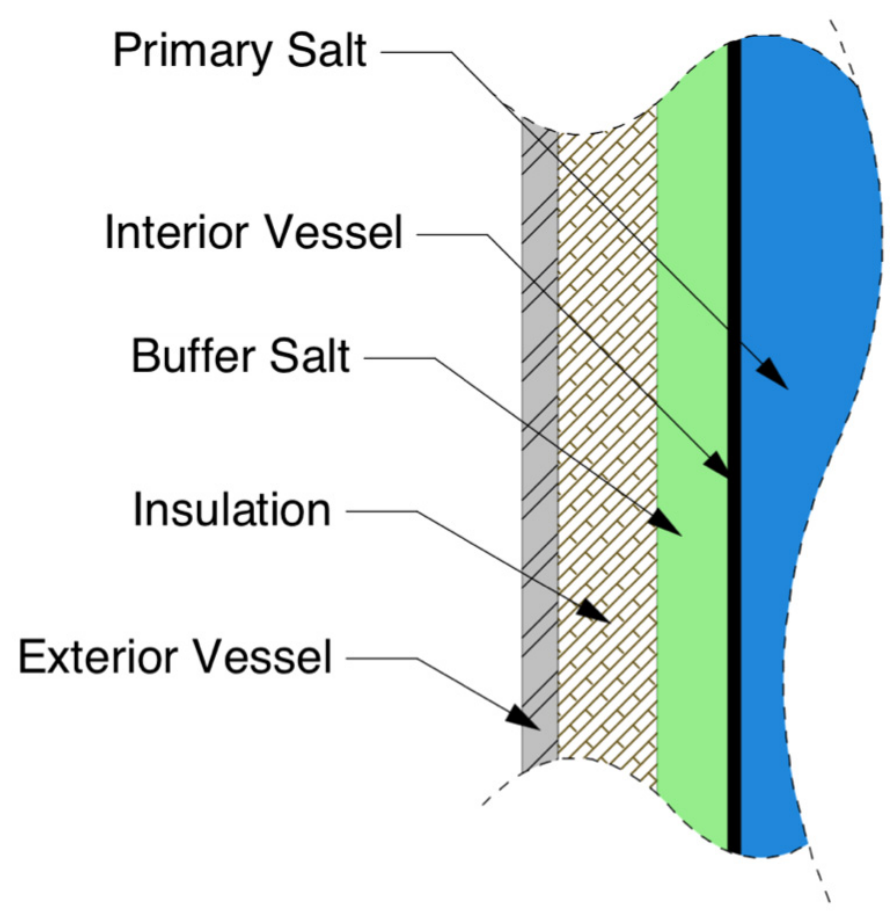

Fig. 10. Reactor vessel layer structure.

\subsubsection{Liquid Salt Pumps}

The primary pump provides pressure to establish an adequate level of forced circulation in the PHTS for effective removal of heat generated by the reactor core. The FHR-TS uses a single primary pump located on the hot leg. Both the hot and cold primary salt legs exit from near the top of the vessel, and the primary salt pump is configured with a vacuum breaker to passively lose siphoning prime upon low reactor vessel salt inventory. The discharge from the primary pump is directed to the IHX. For a $16-\mathrm{MW}(\mathrm{t})$ heat generation rate, the nominal mass flow rate in the primary coolant circuit is approximately $70 \mathrm{~kg} / \mathrm{s}$.

Considerable operating experience exists with liquid salt pumps from the MSRE and MSBR test programs. The primary pump used in the MSRE was a vertical-shaft sump pump with an overhung impeller and an oil-lubricated face seal. More detailed information on the MSRE salt pump design and testing is available as an ORNL technical manual [17]. A cross-sectional drawing of the MSRE fuel salt pump is shown in Fig. 11. It consists of three main components: the pump tank, the rotary assembly, and the drive motor. The main components were bolted together and sealed with oval ring-joint gasketed flanges. The ring-joint grooves were connected to a leak-detection system. The motor and rotary assembly could be removed from the pump tank either as a unit or separately.

The MSRE pump tank provided volume to accommodate the thermally expanded salt of the system. It primarily contains the pump volute or casing, a xenon-removal spray device that can be used for tritium recovery in the FHR-TS, salt level indicators, and various access nozzles. The rotary assembly consists of the bearing housing, the pump shaft, the shaft seals, the shield plug, and the pump impeller. 


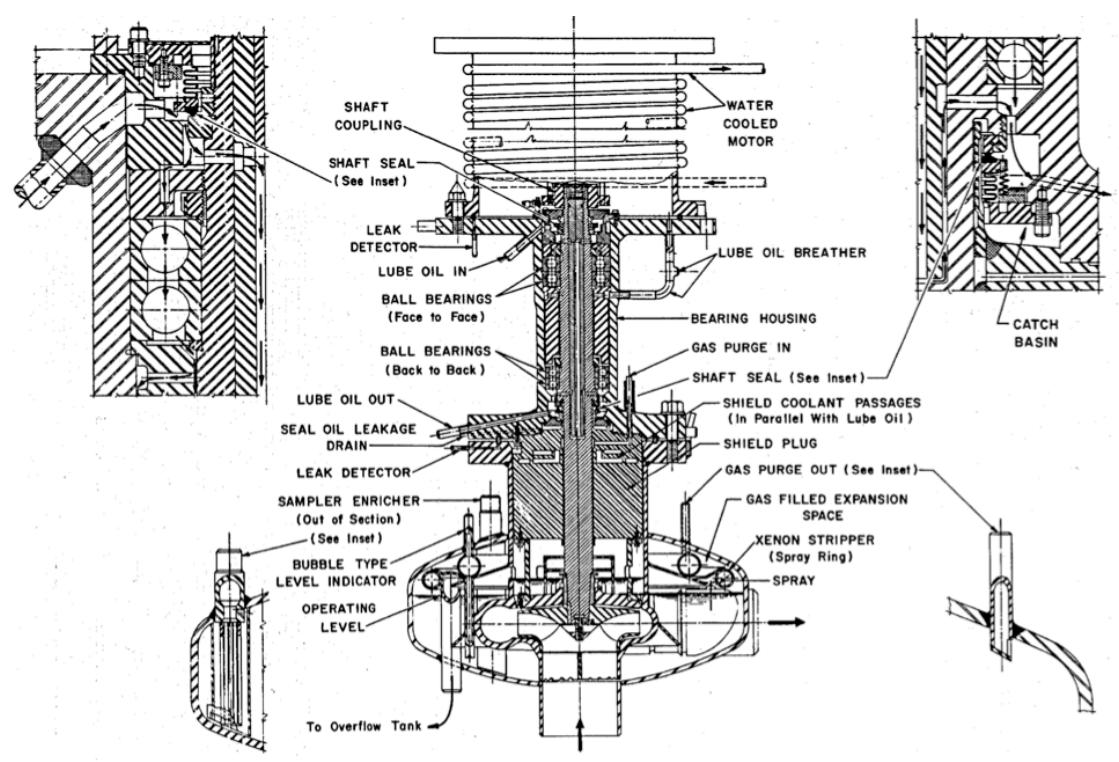

Fig. 11. Cross-section of the MSRE fuel salt pump.

Table 3 shows the design data for the MSRE fuel-salt circulation pump. The pumping requirement for the FHR-TS will be approximately one-half that of the MSRE based on the flow rates.

Table 3. Fuel-salt circulation pump design data for the MSRE

\begin{tabular}{ll}
\hline $\begin{array}{l}\text { Design Flow } \\
\text { Pump Output } \\
\quad \text { Internal Bypass }\end{array}$ & $150 \mathrm{~kg} / \mathrm{sec}(1200 \mathrm{gpm})$ \\
Discharge Pressure & $56 \mathrm{~kg} / \mathrm{min}(65 \mathrm{gpm})$ \\
Intake Pressure & $380 \mathrm{kPa}(55 \mathrm{psig})$ \\
Speed & $48 \mathrm{kPa}(7 \mathrm{psig})$ \\
Design Conditions & $1160 \mathrm{rpm}$ \\
$\quad \begin{array}{l}\text { Pressure } \\
\quad \text { Temperature }\end{array}$ & $345 \mathrm{kPa}(50 \mathrm{psi})$ \\
Motor $\quad$ & $704{ }^{\circ} \mathrm{C}\left(1300^{\circ} \mathrm{F}\right)$ \\
$\quad$ Rating & $56 \mathrm{~kW}(75 \mathrm{hp})$ \\
\hline
\end{tabular}

The primary pump for the FHR-TS will have similar functional requirements. However, certain transients might require that a material with higher approved temperature limit be used. In the current design, the primary pumps are located at the outlet of the hot legs; therefore, they will be subject to the highest bulk coolant temperatures in the loop. Similar design considerations apply to pumps in the intermediate loop. The salt pumps are equipped with thermally activated passive trip systems to shut off the coolant flow in the event of extended shutdown conditions. This protection system will be designed with overcooling transients in mind to prevent freeze-up of the primary liquid salt inventory. 


\subsubsection{Intermediate Heat Exchanger}

The IHX provides an interface between the primary coolant and the intermediate coolant for effective transfer of heat from the PHTS to the IHTS. The IHX is designed to provide adequate static head to establish the driving force for natural circulation in the event of a loss-of-forced-flow event. The IHX also serves as a primary coolant boundary and must be constructed to maintain system integrity under normal, off-normal, and accident operating conditions.

The heat removal requirement per loop for the $16 \mathrm{MW}(\mathrm{t})$ FHR-TS is of similar scale $(2 x)$ to that of the MSRE, but will operate with a significantly lower log-mean temperature difference (LMTD) because it is coupled to a closed gas power cycle rather than a steam cycle. The MSRE IHX was a horizontal, shell and U-tube type, with the fuel salt circulating in the shell and the coolant salt in the tubes. The FHRTS IHX likely must be designed as a single-pass, counter-flow heat exchanger in order to achieve a sufficiently small LMTD, similar to the design adopted for the MSBR. The MSRE heat exchanger was fabricated of Hastelloy-N throughout except for the back-braze alloy used in the tubesheet joints. More detailed design information on the MSRE heat exchangers is available in ORNL-TM-728 [6]. The tube bundle was designed for replacement. Figure 12 shows a drawing of the MSRE heat exchanger, and the design data for the MSRE heat exchanger is given in Table 4.

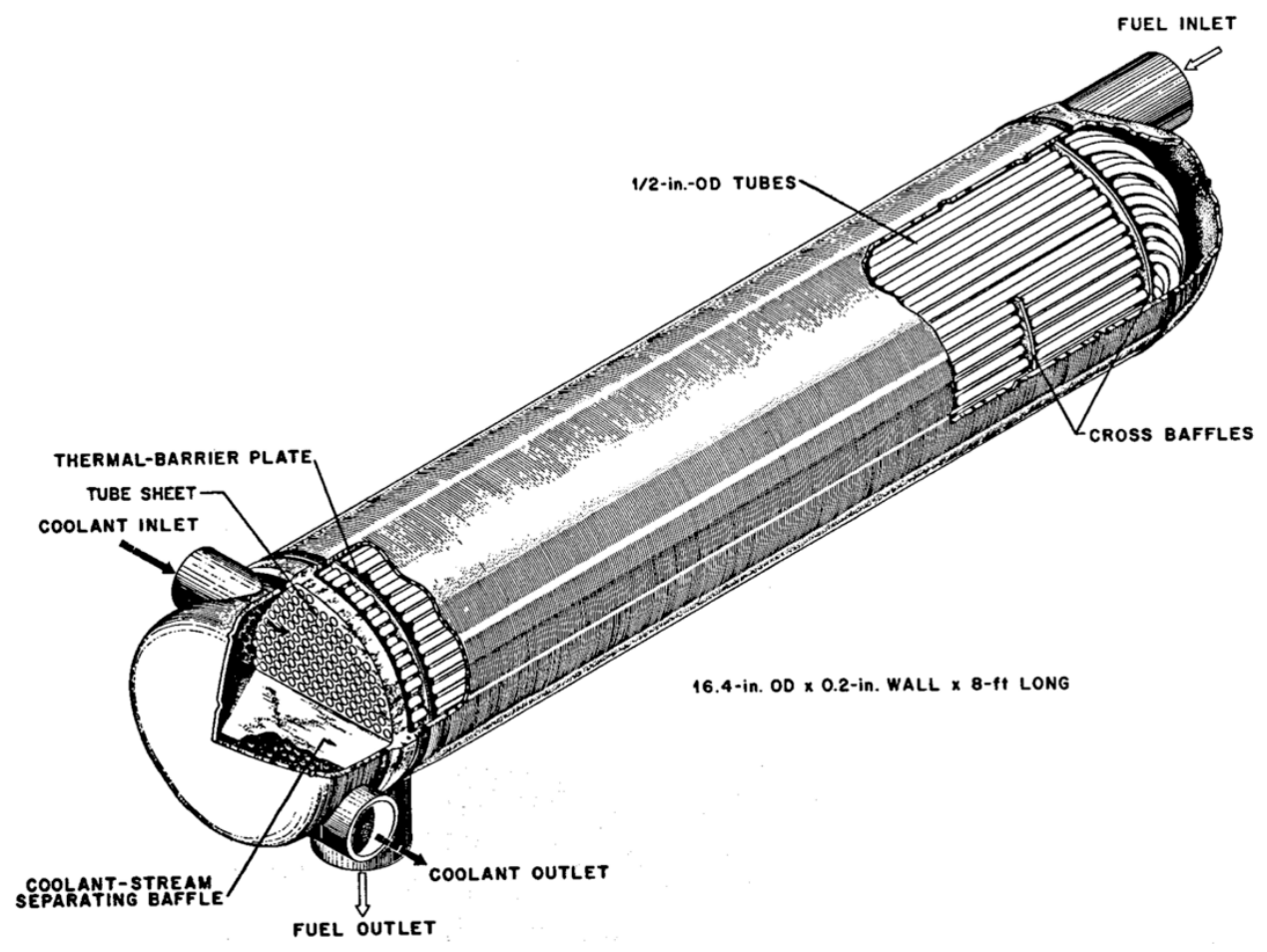

Fig. 12. Heat exchanger for the MSRE.

The MSRE primary heat exchanger was operated with the secondary coolant at a slightly higher pressure than the primary coolant so that the radioactivity would be contained in the primary loop in case a leak develops. In the FHR-TS under power operation the primary coolant is also at lower pressure than the secondary coolant to prevent the spread of beryllium in the primary coolant. Minimizing isotopic contamination of the primary coolant with ${ }^{6} \mathrm{Li}$ thus becomes a primary restriction in the selection of the secondary salt. The operating procedure following tube leak detection involves tripping the reactor and adjusting pump speeds to equalize pressure across the IHXs until the IHTS can be drained and the faulted tube plugged. 
Though shell-and-tube design is the most widely used heat exchanger type across the industry with substantial operating experience, other design types are also under consideration, including plate/fin and micro-channel type heat exchangers. Two major advantages of the shell-and-tube design are that they offer simplified in-service inspection capability if the design allows access to the interior of the heat exchanger vessel, and that they can be more readily designed to minimize steady-state and transient thermal expansion stresses. A single-pass shell-and-tube IHX design, derived from the MSBR IHX, was adopted for the UCB 410-MW(e) PB-AHTR design [18]. The other two designs are generally more compact; therefore, they offer higher power density, which can reduce the initial capital cost.

Table 4. Design data for the MSRE heat exchanger

\begin{tabular}{ll}
\hline Construction Material & Hastelloy-N \\
Heat Load (MW) & 10 \\
Shell-side Fluid & Fuel Salt \\
Tube-side Fluid & Coolant Salt \\
Layout & $25 \%$-cut, cross-baffled shell with U tubes \\
Baffle Pitch (in) & 12 \\
Tube Pitch (in) & 0.775 triangular \\
Active Shell Length (ft) & $\sim 6$ \\
Overall Shell Length (ft) & $\sim 8$ \\
Shell Diameter (in) & 16 \\
Shell Thickness (in) & $1 / 2$ \\
Average Tube Length (ft) & 14 \\
Number of U-tubes & 159 \\
Tube Size (in) & $1 / 2$ OD; 0.042 wall \\
Effective Heat Transfer Surface (ft2) & $\sim 254$ \\
Tubesheet Thickness (in) & $11 / 2$ \\
Fuel Salt Holdup (ft3) & 6.1 \\
Design Temperature & \\
$\quad$ Shell Side $\left({ }^{\circ} \mathrm{F}\right)$ & 1300 \\
$\quad$ Tube Side & 1300 \\
Design Pressure & \\
$\quad$ Shell Side (psig) & 55 \\
$\quad$ Tube Side (psig) & 90 \\
Allowable Working Pressure & \\
$\quad$ Shell Side (psig) & 75 \\
$\quad$ Tube Side (psig) & 125 \\
Terminal Temperature & 1225 inlet; 1175 outlet \\
Fuel Salt ( ${ }^{\circ}$ F) & 1025 inlet; 1100 outlet \\
Coolant $\left({ }^{\circ}\right.$ F) & 133 \\
Effective Log-mean Temperature & \\
Difference ( ${ }^{\circ}$ F) & \\
Pressure Drop & 24 \\
Fuel-salt Flow Rate (gpm) & 1200 \\
Coolant-salt Flow Rate (gpm) & 850 \\
\hline
\end{tabular}




\subsubsection{Secondary Heat Exchanger}

The secondary heat exchanger provides the interface between the intermediate coolant and the Brayton cycle power conversion system. This heat exchanger is designed to transfer the heat from the low-pressure intermediate loop to the high-pressure nitrogen helium mixture or supercritical carbon dioxide working fluid. The heat exchanger serves as the intermediate coolant boundary and must be constructed to maintain system integrity under normal, off-normal, and accident conditions. To maintain high cycle efficiencies, it must also minimize temperature differences between the intermediate molten salt and the power conversion gas working fluid, while minimizing the gas side pressure drop. Since the gas heat transfer is generally low, this usually requires using high gas velocities or high gas side surface areas (enhanced or extended surfaces). The high gas pressure required in the power conversion system (6-10 MPa) also imposes stringent requirements on the heat exchanger design.

Existing NGNP high temperature gas system concepts include a gas-to-liquid salt intermediate heat exchanger to support process heat applications. Both plate-type and printed-circuit-type heat exchanger

designs are being considered for the NGNP application and are also being considered for the liquid-to-gas heat exchanger for the AHTR test reactor as well, along with more conventional tube type designs [19].

Candidate materials of construction include Hastelloy-N and more complex clad designs that enable the use of higher strength and creep resistant alloys. Longer-term ceramic and composite designs may be possible as well. Issues that must be addressed during the design process include materials compatibility with both the intermediate salt and the gas used in the power conversion system, stress levels imposed by both the operating pressures and those thermally induced by the temperature differences that will exist in a heat exchanger of this type, design for acceptable creep over the heat exchanger lifetime, and the fabrication processes needed to manufacture an acceptable design.

\subsubsection{Salt Processing and Inventory Control System}

Long-term operation of the FHR-TS relies heavily on maintaining the purity and low fluorine potential of the fluoride salt. Although high-purity fluoride salts can have low corrosive properties, fluoride salts can become much more corrosive when contaminated by water vapor or oxygen. Other contaminants in the salt could result from corrosion of the pressure boundary material, neutron capture reactions, broken fuel spheres, and graphite dust.

The main function of the Processing and Inventory Control Subsystem (PICS) is to maintain the desired purity and fluorine potential level of the liquid fluoride salt. The PICS also has the capability to transfer salt into and out of a volume control tank to adjust the primary salt inventory. The liquid salt in the PHTS can be pumped from the reactor vessel to storage tanks during maintenance using a gas-lift pump system. Because this operation would only be performed very infrequently, the storage tanks are designed to freeze the salt for removal and storage outside the Reactor Building, using the PICS. To transfer salt from the storage tanks to the primary loop, the tanks are first melted from a free surface and then transferred to the PICS volume control tank using a dip tube and gas overpressure.

The oxygen level in the salt needs to be minimized to reduce the salt's corrosive capabilities. A sidestream flow is diverted from the primary loop into the PICS, which is processed with an $\mathrm{HF} / \mathrm{H}_{2}$ or $\mathrm{NF}_{3}$ purge to remove oxygen contamination. In this purge, fluorine-bearing gas is bubbled through the salt along with hydrogen gas, which chemically reacts with oxygen to produce water vapor, and replaces oxygen with fluorine. For the primary salt, the free fluorine potential is then reduced by flowing the salt past a bare beryllium metal surface. 
The PICS will also be designed to remove other contaminants, including particulates such as graphite dust and larger pebble pieces from broken spheres. The mechanical filter would be fabricated from a corrosion resistant material such as nickel or silicon carbide.

Similar methods are required to provide chemistry control of the buffer salt, the intermediate salt, and the DRACS salt. Because the intermediate salt will not contain beryllium, a different method will be required to adjust the fluorine potential. Depending on the rate of diffusion of tritium through various interfaces, these loops may be required to have tritium control.

\subsubsection{Cover Gas Chemistry and Pressure Control System}

The primary loop cover gas chemistry and pressure control system processes a continuous stream of argon cover gas to sweep tritium, oxygen, and moisture away from liquid salt surfaces and to provide makeup argon flow. The cover gas system provides clean argon flow to purge the shutdown rod activation systems to prevent accumulation of condensed $\mathrm{BeF}_{2}$, and pressurized argon to the pebble transfer system for pneumatic transfer of pebbles.

Tritium is produced by several reactions when using flibe as a primary coolant: ${ }^{6} \mathrm{Li}(\mathrm{n}, \alpha) \mathrm{t},{ }^{9} \mathrm{Be}(\mathrm{n}, \alpha){ }^{6} \mathrm{Li}$ $\rightarrow{ }^{6} \mathrm{Li}(\mathrm{n}, \alpha) \mathrm{t},{ }^{7} \mathrm{Li}(\mathrm{n}, \alpha \mathrm{n}) \mathrm{t}$, and ${ }^{19} \mathrm{~F}\left(\mathrm{n},{ }^{17} \mathrm{O}\right) \mathrm{t}$. The molten salt breeder reactor design estimate was that at a $99.995 \%{ }^{7} \mathrm{Li}$ purity in the primary coolant, roughly $1 \mathrm{Ci}$ of tritium would be produced per day per megawatt (thermal) of reactor size [20]. Tritium and ${ }^{6} \mathrm{Li}$ production have been calculated for a 2400 $\mathrm{MW}(\mathrm{t})$ AHTR configuration [21]. The equilibrium ${ }^{6} \mathrm{Li}$ concentration in the coolant is $0.001 \mathrm{wt} \%$, or about $10 \%$ of its initial concentration. This is due to the burnout of the initial ${ }^{6} \mathrm{Li}$ loading balanced by the generation of new ${ }^{6} \mathrm{Li}$ from Be reactions. At this equilibrium concentration, tritium production is $\sim 500 \mathrm{Ci} /$ day. The time required for the salt to reach the equilibrium ${ }^{6} \mathrm{Li}$ concentration will depend upon the salt inventory, but may be between 18 months and a few years. The initial tritium production will be on the order of $5000 \mathrm{Ci} /$ day, dropping to approximately $500 \mathrm{Ci} /$ day at equlibrium. For comparison, an LWR of comparable thermal power produces $\sim 50 \mathrm{Ci} /$ day, and a typical heavy water reactor (HWR) produces 3500-6000 Ci/day. The largest fraction of the created tritium will become as a dissolved species in the primary salt. Almost all of the remainder will be trapped in the nearby core graphite.

The approach used at MSRE to control the created tritium was to sweep the tritium from the primary salt by bubbling helium through a side stream of primary coolant and chemically trapping the entrained hydrogen isotopes. Ultrasonic degassing is an alternate approach to removing dissolved tritium that has seen significant development since the cancellation of the MSR program. Minimizing the tritium content in the primary loop avoids significantly contaminating the secondary loop (tritium permeability of the intermediate heat exchanger will be a significant design parameter). In general, all cover gas systems will require chemical trapping of the hydrogen isotopes prior to release.

\subsubsection{Valves for Liquid Salts}

While the PHTS and IHTS will both use variable speed pumps for flow control, additional means of controlling the flow of liquid salt will still be needed. Needs range from isolation of flow during fill and drain activities to controlling flow during normal, shutdown, and off-normal operation of the primary and intermediate heat transfer circuits and other supporting molten salt systems.

The control strategy for operating the plant during start-up, steady-state operation, shutdown, and other off-nominal conditions has not been firmly established. It is likely that most salt circuit flow control will be accomplished using variable speed pumps. However, for certain operating regimes or under certain conditions where further flow restriction is necessary, valves can be used for throttling purposes. 
The current FHR-TS plant concept incorporates containment isolation valves in the intermediate salt lines.

\section{Mechanical Valves}

In most circulating fluid systems, mechanical valves are used extensively. Challenges to the use of mechanical valves include general material compatibility in a fluoride salt environment and at FHR-TS operating temperatures. Fluoride salts are excellent fluxing agents, and thus self-welding and galling are important design issues for valve seats and actuator seals.

To date most fluoride salt circulating systems have avoided the use of mechanical valves. Freeze valves (discussed below) have been used, or the system is designed to operate without valves (such as controlling flow by adjusting pump speed rather than through a control valve). For common reactor applications such as containment isolation, however, mechanical valves may be needed to meet performance requirements such as actuation time or capacity to isolate larger diameter pipes.

\section{Freeze Valves}

An alternative to the mechanical cutoff valve is provided by a freeze valve. In a freeze valve, salt contained within a flattened section of pipe is either cooled below its freezing temperature by a stream of air or other coolant, or is melted by electric heaters. Freeze valves were used on the 4-cm-diam drain lines in the MSRE; an example is shown in Fig. 13. Extensive experience has been obtained with freeze valves, and so long as the stress produced in thermal cycling during freeze-thaw operation is properly managed, these valves have performed well.

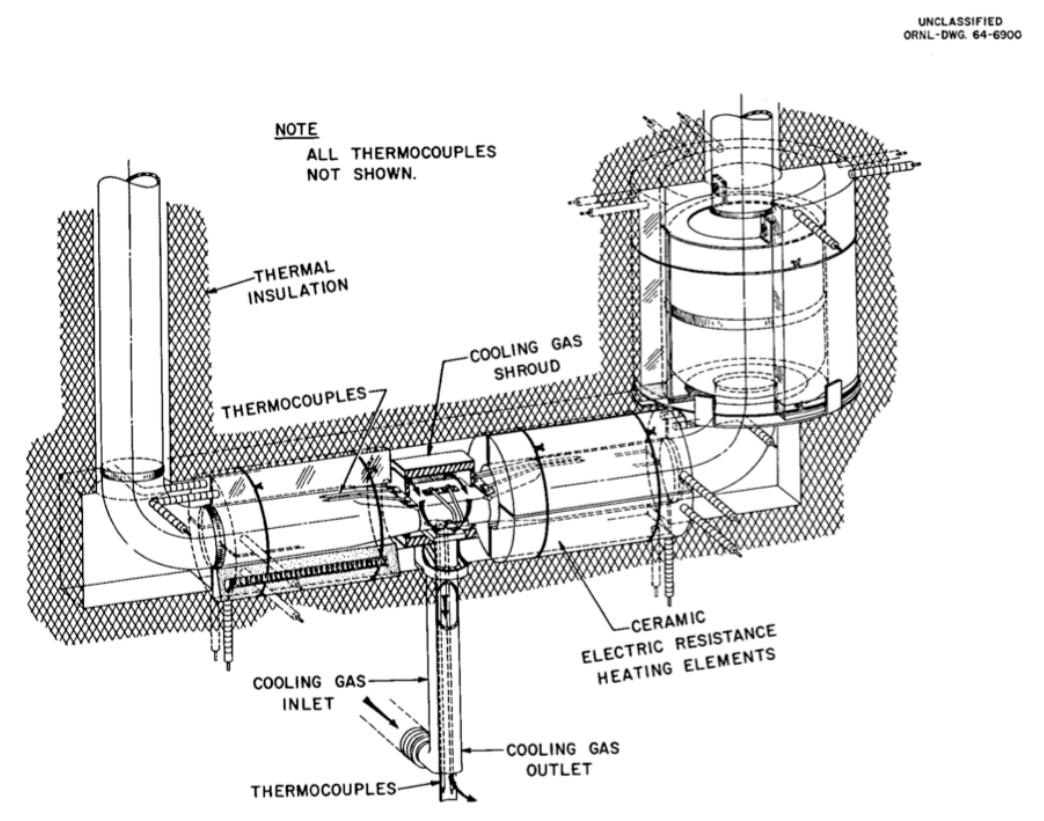

Fig. 13. Freeze valve design from the MSRE.

A freeze valve consists of a flattened section of pipe, cooled at the center by an insulated air stream, and a heated furnace, which encloses the entire valve. This arrangement controls the manner in which the salt freezes and thaws so as to prevent damage resulting from trapped liquid expansion during the thaw. Possible modes of operation for freeze valves are (1) open, (2) closed but ready to open on a power failure, (3) closed and remain closed on a power failure, and (4) closed but ready to open rapidly $(<15 \mathrm{~min})$ on demand. The time required to freeze a valve varies from 5 to $30 \mathrm{~min}$ depending on the 
starting temperature, and the time required to thaw varies from less than $15 \mathrm{~min}$ up to several hours depending on the mode of operation at the time of the demand.

Freeze valves are applicable to shutoff and isolation applications, where the times to close and open are consistent with operational needs. Establishing a freeze plug requires a static salt inventory in the freeze section of the valve. This can be accomplished by stopping flow in a filled line, or by retaining a sufficient volume of salt after a salt transfer is complete. The pot shown in the figure is to retain a sufficient inventory of salt to establish a frozen plug; once the flow of gas used to complete a transfer of salt ceases, the salt in the pot flows back into the low-elevation portion of the valve.

Freeze valves are expected to be used in the FHR-TS to isolate systems associated with filling and draining the reactor and heat transfer circuits, and at other locations where isolation of partial systems may be needed to support maintenance.

\subsection{DIRECT REACTOR AUXILIARY COOLING SYSTEM}

The DRACS provides the primary means for removing decay heat from the reactor core in the event of failure of the primary and shutdown cooling systems. The DRACS concept was originally developed for the Experimental Breeder Reactor II (EBR-II) and has been used in a number of reactor designs. DRACS are modular, natural circulation heat transfer loops with the energy source being the primary coolant and the energy sink being ambient air. Energy is transferred from the primary coolant into the DRACS via an in-vessel natural circulation loop. Thermal energy is then transferred from the in-vessel loop to an external cooling loop via a natural draft heat exchanger. An illustration of the relationship of the DRACS and the primary heat transfer circuit is shown as Fig. 14.

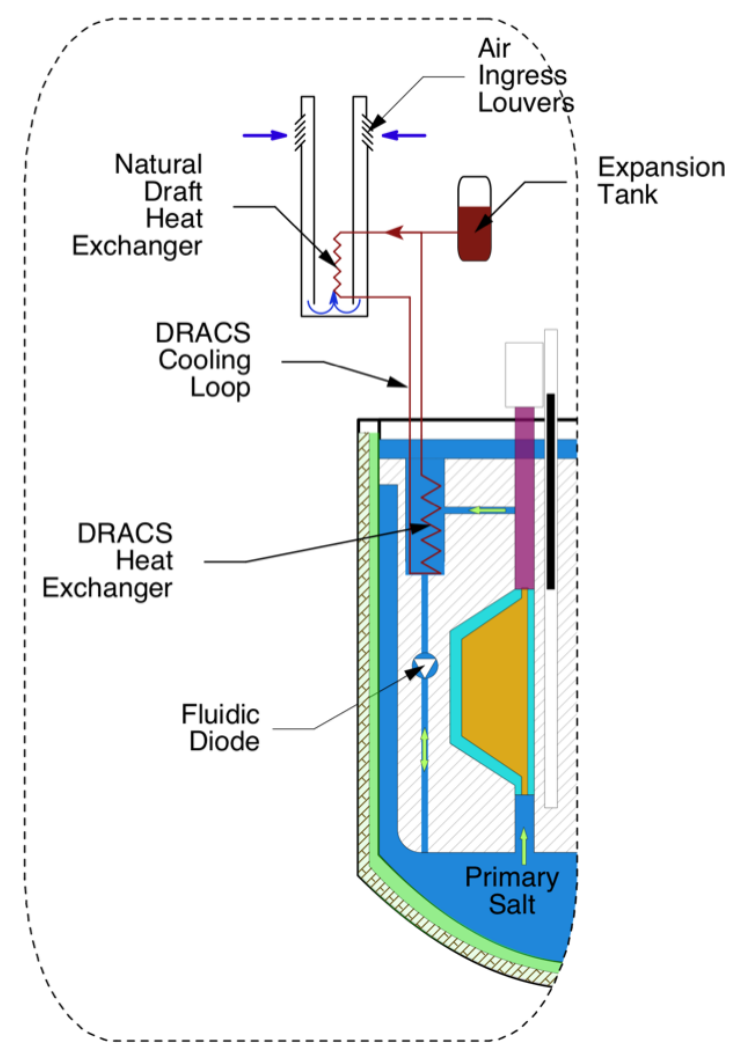

Fig. 14. Schematic drawing of DRACS and its interface with the Primary Heat Transport System. 
Excess energy transfer to the DRACS during normal power operation is avoided by placing a fluidic diode oriented to oppose the primary coolant pump on the downward flow leg of the in-vessel natural circulation loop. The small residual reverse flow though the fluid diode is scaled to provide sufficient energy to maintain the DRACS cooling loop in a liquid state. DRACS used for sodium fast reactors typically use $\mathrm{NaK}$ as a low-melting point coolant; however, for the application to the FHR the use of chemically-reactive $\mathrm{NaK}$ would introduce a source of stored chemical energy that would then necessitate the use of a high-pressure containment structure. Thus the leading candidate materials for the DRACS

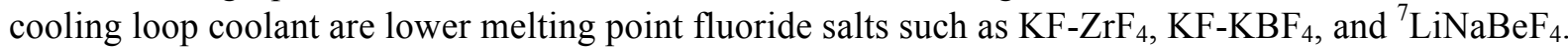

Principal components of the DRACS are in-vessel DRACS Heat Exchangers (DHX), the fluidic diode, a secondary Natural Draft Heat Exchanger (NDHX), an expansion tank, and an exterior stack that forms the natural draft pathway for dissipating the decay heat to the atmosphere. The system operation solely depends on natural convection. The airflow into the NDHX is regulated by a louver system to control the DRACS heat removal rate and to control overcooling transients.

For the baseline calculations, the DRACS was sized to be capable of removing 5\% of the FHR-TS rated power. The actual capacity will be determined subsequently using detailed transient modeling for the thermal response of the reactor to loss of forced circulation and other design basis transients and accidents. In a test scale reactor the required loss of forced flow heat rejection will likely occur naturally via natural conduction and convection to the surrounding environment. Thus the primary purpose for a DRACS in a test reactor is to demonstrate system characteristics to support larger scale deployment. The entire heat rejection capability for the FHT-TS is thus intended to be provided by a single unit, whereas in larger reactors (in which the DRACS performs a safety function) the heat rejection capability would be distributed among multiple, redundant DRACS units.

The DHX provides an auxiliary heat removal mechanism transporting heat directly from the primary liquid salt to a separate DRACS coolant contained within the DHX boundary. The primary liquid salt is the working fluid on the shell side, and the DRACS coolant is the working fluid on the tube side. The DHXs do not have any active components and solely depend on natural circulation.

The NDHX natural draft heat exchanger transports heat removed by the DHX working fluid from the primary liquid salt to the atmosphere. The NDHX is located at a sufficiently high elevation to ensure adequate head to drive the natural circulation mode under the predetermined operating conditions [22].

The NDHX is located at the bottom of a reinforced concrete chimney. The air intake is located high on the chimney, and air flows down the chimney in an annulus around an insulated metal duct in which heated return air flows upward. As an exterior safety component, the DRACS chimney structure needs to be fabricated to be resistant to external missiles. The down flow of intake air maintains the concrete at low temperature near the external ambient temperature. The air intake openings employ a passive controller that is directly coupled to the primary coolant pump(s). Mechanical coupling can be designed in the form of a vacuum formation to keep the louvers closed under normal operating conditions. In the event of loss of forced circulation, loss of vacuum will result in opening of mechanical louvers to allow air intake. Loss of electrical power to the pumps will also release the electromagnets holding the springloaded louvers shut. Both passive control mechanisms can be used to provide redundancy.

Overcooling transients are a potential long-term FHR accident. Therefore, the DRACS air intake system also needs to employ passive mechanisms to shut the mechanical louvers in the event that the primary coolant temperature is reduced below a fixed setpoint (roughly $100^{\circ} \mathrm{C}$ above its melting point). The lower temperature threshold of air intake closure is a function of DRACS heat removal capacity and will be determined by coupled thermal-hydraulic simulations of the PHTS and DRACS with decay heat generation by the reactor. 
To prevent the DRACS from parasitically rejecting heat during normal operation, a fluidic diode is included in the primary coolant in-vessel natural circulation flow path as shown in Fig. 14. Fluidic diodes are passive flow control devices with low flow resistance in one direction and high flow resistance in the opposite direction. In this regard, a fluidic diode works in a similar fashion to a check valve. The forward flow resistance of the fluidic diode is a passive safety-related component necessary to reject reactor decay heat in the event of a loss of forced flow coupled with loss of shutdown cooling. There have been different fluidic diode designs proposed in the literature. A few potential candidate designs are introduced below.

One potential fluidic diode design employs an expansion chamber containing a multitude of balls and an inlet and outlet screen of different size (Fig. 15). When the balls pile up against the smaller screen, they provide high flow resistance. However, an insufficient number of balls exist in the expansion chamber to block the larger screen. Figure 15 (a) and (b) illustrate the behavior of the device under low and high flow-resistance conditions, respectively.

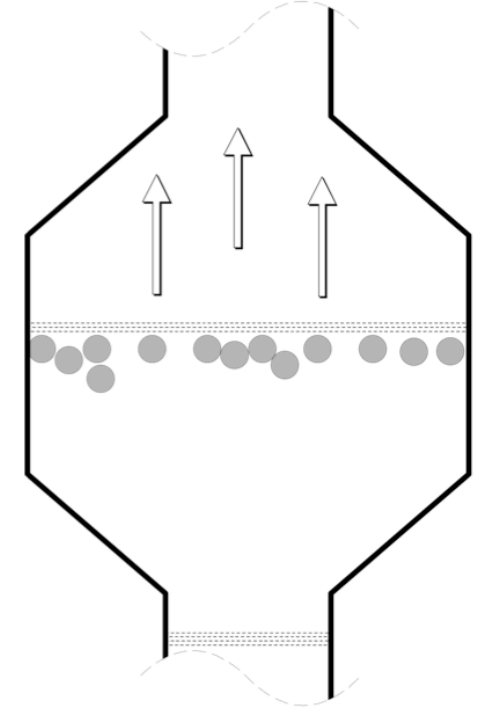

(a)

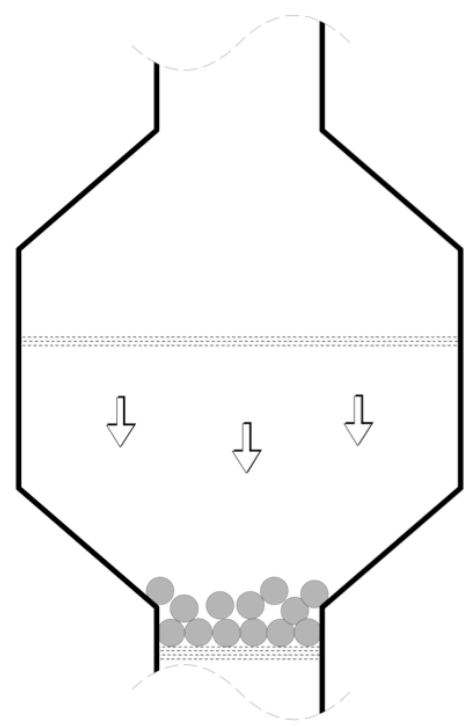

(b)

Fig. 15. Simplified schematic of floating ball type fluid diode: (a) low flow-resistance condition, (b) high flow-resistance condition.

Vortex diodes, as shown in Fig. 16, are leaky flow control devices used to provide unidirectional flow in situations where it is desirable to avoid valves with moving parts. In principle, a vortex diode generates an irreversible loss of kinetic energy by creating a strong vorticity in one direction, while flow in the opposite direction does not have this effect. The basic design of a vortex diode consists of a discshaped chamber with cylindrical axis and tangential ports. The flow entering the device through the tangential port sets up a vortex, which induces a large pressure drop in the flow path. Flow through the axial port creates a predominantly radial flow distribution over the chamber cross section, which develops only a modest pressure drop [23]. 


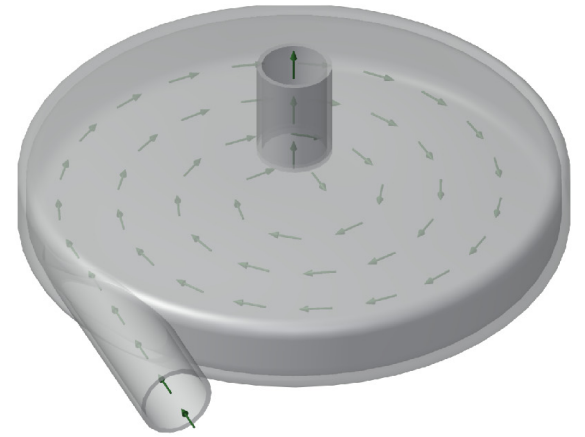

(a)

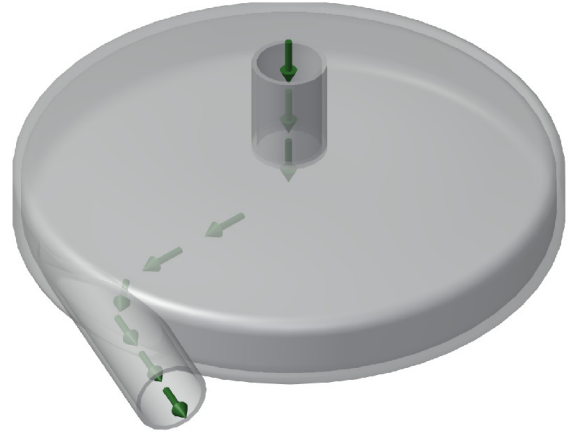

(b)

Fig. 16. Vortex type fluid diode: (a) reverse flow (high resistance), (b) forward flow (low resistance).

An alternative fluidic diode design also based upon fluid flow angular momentum was proposed and demonstrated by Rothfuss et al. [24], as shown in Fig. 17. This design employs a series of bent blades that guide the flow. As shown in the figure, the flow in the upward direction goes through the impeller and develops a rotational component as it exits. Guided along each blade, the fluid experiences a cascade of pressure drops as it progresses throughout the device. The flow on the opposite direction, however, has less resistance and develops much less pressure drop because of a larger fraction of axial velocity. The ratio of pressure drops, which is defined as diodicity, can be designed to exceed 50 .
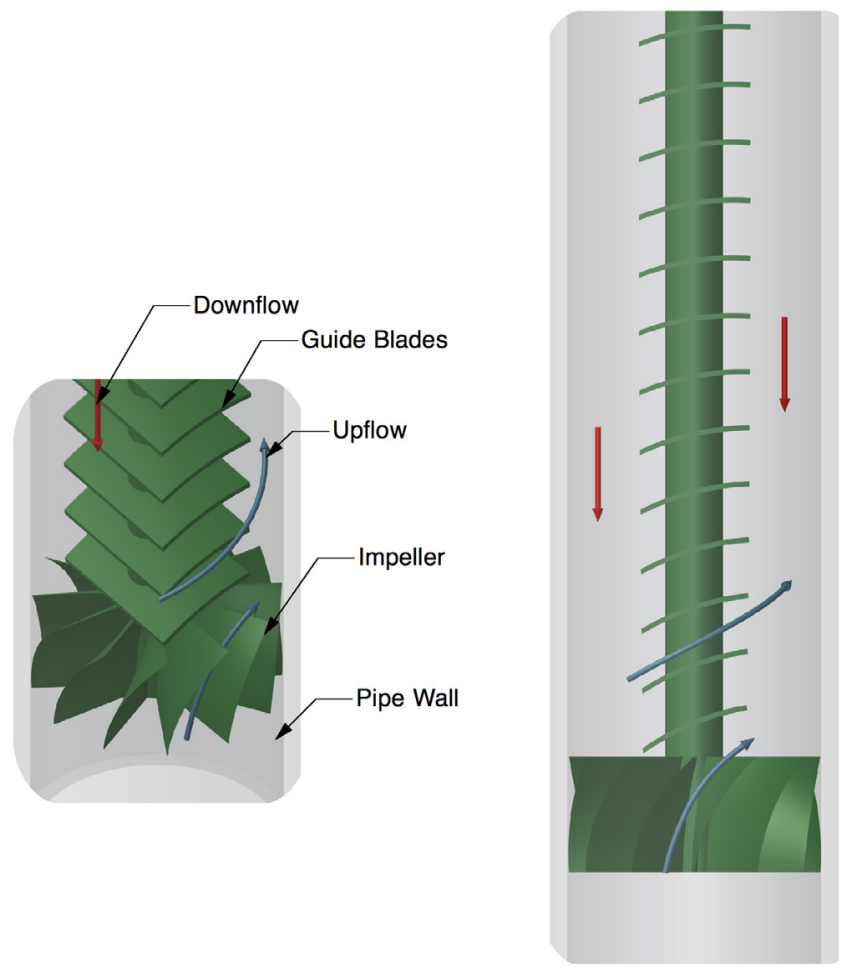

Fig. 17. Fluidic diode design based on guide blades. 


\subsection{INSTRUMENTATION AND CONTROLS SYSTEM}

The Instrumentation and Controls (I\&C) system consists of safety-related and non-safety-related components to perform automated plant protection, control and monitoring functions, and provide interfaces for operator-to-plant interaction, i.e. Human-System Interfaces (HSIs). The FHR-TS will employ passive mechanisms to perform the key safety functions such as reactivity control, decay heat removal, and coolant inventory control.

The operational instrumentation requirements for FHRs differ from those for water-cooled reactors primarily in that the transducers need to survive the high-temperature, fluoride salt environment. Apart from the transducers, FHR instrumentation closely resembles that for any other modern nuclear power plant. This technology evaluation will therefore be limited to the distinctive transducers and control requirements of the FHR.

FHR development and operation require measurement of a broad set of process variables including temperature, neutron flux, flow, and level. Coolant chemistry measurements (as a corrosion indicator) and component health monitoring are also important for longer-term operation. For most process variables, several alternate measurement approaches are possible. This report includes overview descriptions of the principal candidate measurement approaches. However, a more comprehensive instrumentation evaluation task is recommended to capture the technical details of all the potential measurement approaches.

Temperature measurement is both indicative of process conditions as well as a primary component of the energy transfer measurements necessary for efficient power plant operation. Thermocouples are the most common transducer for process condition measurement. However, base metal thermocouples lack the long-term accuracy necessary for the heat balance measurements necessary for efficient electricity generation. Two optical instruments potentially have the stability and accuracy required to support efficient electricity generation. Both fiber optic coupled pyrometry and Fizeau cavity type thermometers are candidate technologies for high-accuracy temperature measurement. Ultrasonic wireline thermometry is also a strong candidate technology for low-uncertainty temperature measurement at elevated temperatures.

As with HTGRs, the high temperatures of FHRs are above the temperature tolerance of commercially available fission counters and ion chambers requiring potentially complex active cooling for these instruments. However, with improved materials the technology of fission chambers may be adaptable to the required temperature environment. Gamma thermometers with high temperature tolerant materials are the leading candidate technology for FHR local flux mapping.

Liquid salt flow measurement will most likely either be performed using external, ultrasonic flowmeters or Venturi-type flowmeters that use differential pressure gauges as their active element. Ultrasonic flowmeters are currently gaining wide acceptance in LWRs as a primary coolant flowmeter due to their low uncertainty and stability. The high temperature of FHRs requires the use of mechanical stand-offs to limit the ultrasonic transducer temperature exposure. The electronics for water-cooled and salt-cooled reactor ultrasonic flowmeters would be essentially identical. The differential pressure gauges required for Venturi base flow measurement either require diaphragm deflection measurement tolerant of FHR temperatures or impulse line interconnection between a high temperature and a low temperature diaphragm, which would be instrumented with conventional low temperature diaphragm deflection technology. Optically based diaphragm deflection measurement is the leading candidate for direct, high temperature implementation. 
Several technologies are available for FHR salt level measurement. Bubbler-type level measurements based upon the pressure required for minimal flow in a vertical tube are commercially available technology. Also, radar-type level measurements based upon reflection off the top surface of the salt are commercially available. The radar gun and electronics would be located in a standpipe above the fluid well outside of the high temperature and high radiation zones. Mechanical float-type level measurements can also be readily adapted to the FHR by attaching a mechanical extension to a float on the surface of the salt. The mechanical extension would be configured such that it would extend into a nonmetallic standpipe above the vessel enabling the position of the end of the mechanical extension to be determined magnetically.

Because the FHR test reactor will be heavily instrumented, it is expected that it will be designed to accommodate a larger number of instrument types than would ultimately be used in commercial FHR plants. 


\section{SYSTEM MATURITY EVALUATION}

\subsection{SUMMARY}

This section presents an evaluation of the maturity of the FHR-TS systems and components described in the previous section. At the present state of reactor design, much of the specific technology to be applied to the FHR-TS remains undetermined. Consequently, the maturity evaluation remains at a higher level of abstraction, describing previous and similar applications of salt-cooled reactor relevant technology applicable to almost any salt-cooled reactor and does not attempt to provide design-specific technology evaluations.

\subsection{REACTOR CORE SYSTEMS}

\subsubsection{Fuel Elements}

The neutronic aspects of fuel elements are outside the scope of component testing. However, the thermal, mechanical, and hydraulic aspects of fuel elements are part of the primary heat transport system. The flow resistance and heat transfer within an idealized bed of spheres are well known. Flow patterns and heat transfer behavior inside the shaped annular flow region of the pebble bed AHTR are topics for further investigation as well as the control of pebble location within the bed if pebble segregation is needed within the core region. The structured motion of the ensemble of pebbles within the primary, with hydrodynamic forces, circuit has yet to be fully demonstrated even with surrogate materials. Less well known is to what extent the fuel pebbles remain pristine spheres as they are irradiated and mechanically wear during use. Also, pebble tribology changes over their lifetime remain largely unknown.

Mechanically induced surface roughening during use could potentially alter a pebble's heat transfer performance. Methods to clean salt from pebbles after defueling also require study. The freeze and thaw of salt on the surface of a pebble when it is extracted and inserted into the salt loop may cause surface pitting. Changes in the surface condition of the fuel elements may also potentially alter the movement patterns of fuel within the core impacting the system nuclear performance. Also of concern is the potential loss of pebble buoyancy due to coolant density decrease during a beyond design basis accident. While the resulting core separation would decrease the system reactivity, potential core motion requires careful investigation.

\subsubsection{Reactivity Control and Shutdown System}

The specific interrelation between reactivity control methods has not yet been defined. The primary means for adjusting the core reactivity is anticipated to be continuous addition and removal of pebbles from the reactor core. The rate of pebble exchange required will be most strongly influenced by the reactivity insertion rate necessary to overcome xenon transients during power shifts. Flow rate reactivity control is also possible for the FHR-TS. FHRs have strong negative thermal reactivity feedback, excellent single-phase heat transfer, and robust fuel, making coolant flow velocity a potential control technique. The fuel insertion and removal rate requirements may be reduced if the primary pump speed can also be employed as a control mechanism.

Control and shutdown rod hardware may be relatively traditional. The kinetic behavior of the graphite-moderated, thermal reactor is expected to be consistent with experience in many other reactors, and the design of absorbers is likely to be fundamentally similar. One key difference is operating temperature; this will impose requirements for high temperature materials and efficient component 
cooling. A second difference is compatibility with the salt coolant. If the control elements operate in a closed guide tube, long-term salt compatibility requirements apply to the guide tube only (although materials that can withstand a salt environment in the short term would guard against control rod failure in accident scenarios that lead to salt leakage into the guide tube). With a sealed guide tube, cooling of control rod elements would require an internal cooling system. If the control rod were to operate in the coolant salt flow, all salt-contacting materials used in the control rod assembly would need to be qualified for long-term exposure to salt at operating temperatures.

Heat is generated in the absorber elements. The FHR-TS is expected to operate with a coolant exit temperature above $700^{\circ} \mathrm{C}$. Unless a significant and safety-related internal control rod cooling system is provided, this will serve as the ambient temperature above which the control rods must operate. Nickel alloys are often used for compatibility with the salt. Nickel absorbs neutrons; this affects neutron economy and the worth of the absorbers inside a guide tube, and raises the temperature of the guide tube (and the ambient temperature of components inside the tube). An assessment of the neutron absorber element temperature has not yet been performed; temperatures approaching $1000^{\circ} \mathrm{C}$ are plausible. The use of high temperature materials such as carbon/carbon $(\mathrm{C} / \mathrm{C})$ or silicon carbide/silicon carbide $(\mathrm{SiC} / \mathrm{SiC})$ composite materials may be considered for control rod cladding, absorber supports and flexible guide joints, and control rod guide tubes. Shutdown absorber systems should be designed to operate with high reliability, preferably utilizing intrinsic features of the reactor system. Specific concepts, operating features, and mechanical components have not yet been defined. While conceptually simple, poisoned salt injection modules have never been demonstrated with prototypic materials. The conceptual system design would closely follow Kambe and Uotani's analysis [12] albeit with different materials. Removal of pebbles from the core can be considered as an ultimate shutdown mechanism.

\section{Operating experience with salt-cooled reactors}

Many of the above requirements were met in the design and operation of the MSRE. The primary salt served both as fuel and coolant; small additions of enriching fuel were made periodically to counter burnup of fuel in the salt. Continuous assessment of core reactivity was used to plan for additions of enriching fuel salt. Other than burnup, no direct removal of fuel from the reactor was performed during operation. Fuel, dissolved in the salt, could be drained from the reactor by thawing a freeze valve under the reactor vessel. This served as an ultimate shutdown mechanism and allowed for maintenance of reactor components after shutdown.

The MSRE control and shutdown assemblies are shown in Fig. 18. The absorber assembly was contained within a Hastelloy-N guide tube; sections outside the core were Schedule 40 pipe, and the section in the core was tubing. The core exit temperature was $654^{\circ} \mathrm{C}$, slightly below the exit temperature of the FHR-TS. The absorber elements were constructed of gadolinium oxide and aluminum oxide, and are driven into the core on a flexible shaft. A common flange was used for all three absorber assemblies. The primary reactor control feature was the strong temperature coefficient in the liquid fuel, and no criterion for fast operation was imposed on the physical control assemblies. The absorbers were cooled by an internal air-cooling system. Further descriptions of the MSRE control assemblies are given in ORNL-TM-728 [6].

The earlier Aircraft Reactor Experiment (ARE) also used fuel dissolved in molten salt. In the case of the ARE, the fuel salt was contained in tubes, and most of the reactor hardware was immersed in and cooled by liquid sodium. The ARE was a fairly simple test and was not designed for long-term operation. Some of the control experience with the ARE may be applicable to the FHR-TS. Experience with other high temperature sodium-cooled reactors may also be applicable. 


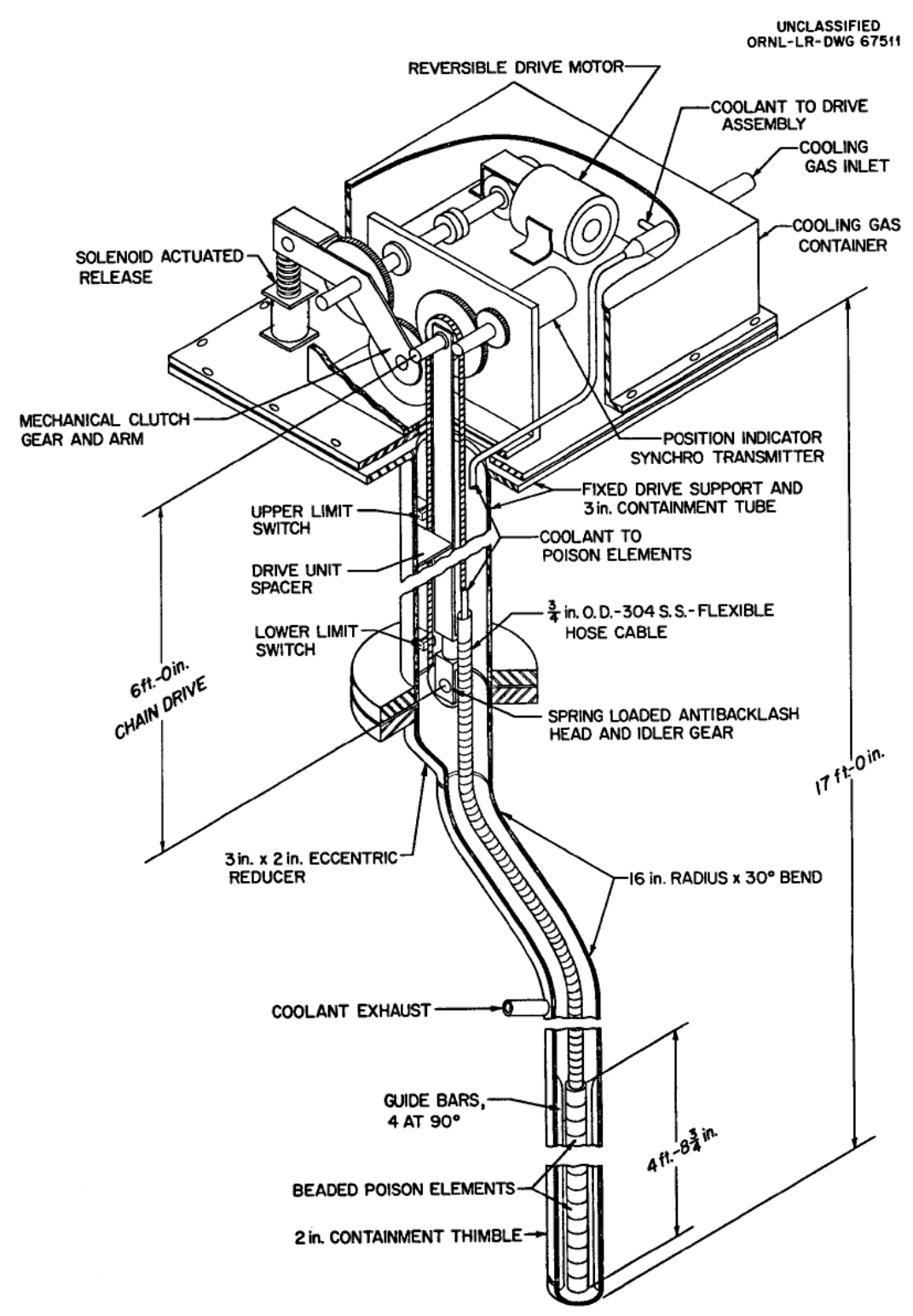

Fig. 18. MSRE control and shutdown assembly.

\section{Operating and test experience with gas-cooled pebble bed reactors}

Many of the potential features for the FHR-TS also appear in existing or proposed high temperature gas-cooled reactors. For example, the preliminary description document for the pebble bed modular reactor (PBMR) design and heat removal describes a reactivity control system based on control rods and small absorber spheres, both using $\mathrm{B}_{4} \mathrm{C}$. Borings in the outer reflector hold both chain-driven control rods, while others are filled with absorber spheres.

\subsubsection{Fuel Handling and Storage System}

A number of gas-cooled reactors have operated on pebble fuel. The AVR and THTR-300 both operated in Germany. The HTR-10 is currently operating in China, largely modeled after the AVR. 
Several high temperature gas-cooled pebble bed reactors are now being constructed in China. The PBMR is being developed in South Africa, with international partners. Since these are all gas-cooled reactors, the pebble fuel is discharged in a gas-cooled environment and all pebble handling, transfer, and storage activities (aside from introduction of fresh pebbles) are conducted remotely using pneumatic systems.

It is expected that the pneumatic portion of the FHR-TS FHSS will be similar to the transfer systems that have been developed for helium-cooled pebble bed reactors such as the HTR-10 and the PBMR. There are key differences in design parameters as well as functional requirements for a salt-cooled reactor:

1. The FHR-TS pebble diameter is smaller than the PBMR or HTR-10 pebble diameter. Currently, a $3-\mathrm{cm}$ fuel pebble diameter is chosen as the design parameter, although not finalized. The PBMR and the HTR, on the other hand, use 6-cm-diam fuel pebbles.

2. Both the HTR-10 and the PBMR are highly pressurized systems. Therefore, the fuel handling system also operates at high pressures, resulting in a more complex design. The FHR-TS, however, operates only slightly above atmospheric pressure.

3. The PBMR fuel handling system loads fresh fuel from the top of the core, and pebbles are discharged from the bottom of the reactor core. In the FHR-TS baseline design, the flow of pebbles is reversed. However, because the FHR-TS primary vessel is not designed to have any penetrations in the lower section, the pebbles need to be injected into the inlet coolant flow and carried to the bottom of the pebble bed.

4. The coolant in the PBMR is helium; therefore, fuel pebbles do not need any cleaning once they are discharged from the core. However, in the FHR-TS, fuel pebbles are likely to have salt residue on them upon extraction. Any residue on the pebbles must be cleaned before they are sent to temporary storage.

The general approach to adding or removing pebbles from the FHR-TS is conceptually similar to the gas-cooled pebble bed reactors, but the salt environment results in several key differences. Pebbles float in the salt coolant; they are introduced to the bottom of the core and removed from the top. An early proof-of-principle Pebble Recirculation Experiment (PREX-1), built at the University of CaliforniaBerkeley, was used to evaluate the general concepts of handling pebbles that float in the coolant. This facility, shown in Figs. 19-20, uses water as a surrogate fluid, with simulated pebble material selected to provide the appropriate buoyancy. This type of test can be used to demonstrate basic pebble handling in a liquid environment.

The FHR-TS design is also based on radial zoning of pebbles. Fuel pebbles are at the center of the core annulus. Graphite reflector pebbles are positioned between the fuel pebbles and the solid graphite reflector. The PREX-3 test, shown in Fig. 5 is a dry, $30^{\circ}$ test demonstrating the layering of pebbles in a partial mockup of a $16 \mathrm{MW}(\mathrm{t})$ reactor core. Because this is a dry test, pebbles are introduced at the top and exit at the bottom. This test is being used to confirm the motion of pebbles through the core, and to show that radial-zoning of pebbles is feasible. 


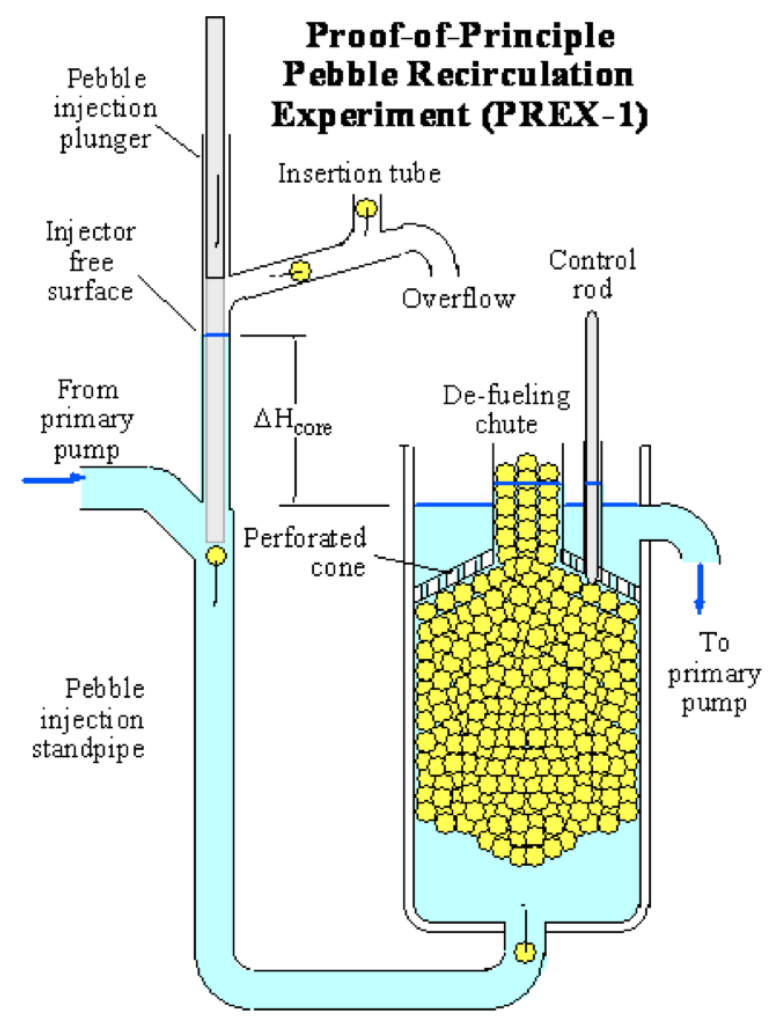

Fig. 19. Test of fuel pebble handling proposed for the PB-AHTR. (Courtesy of University of California-Berkeley)

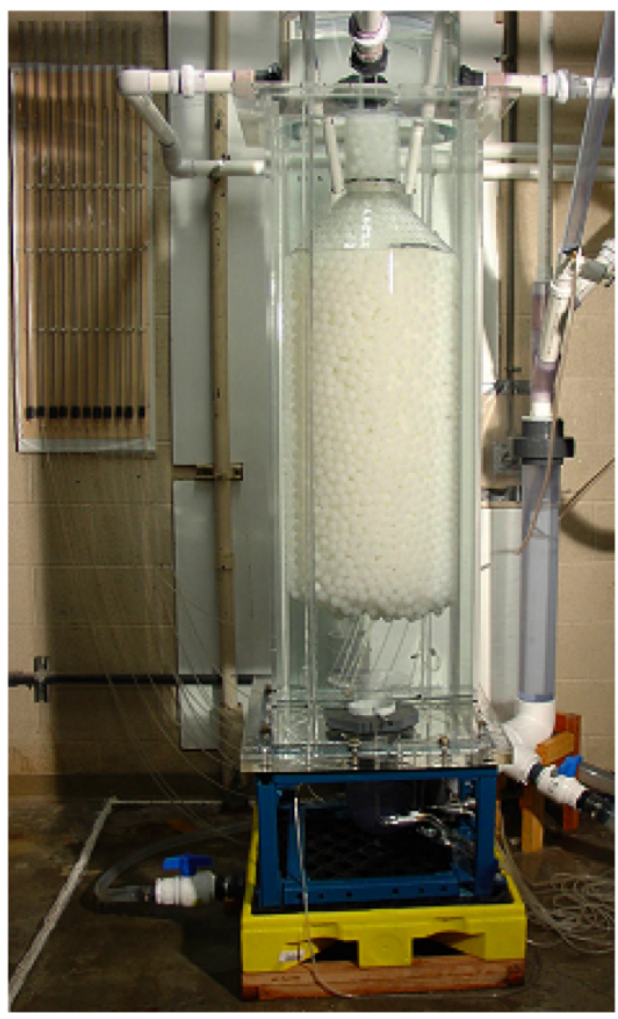

Fig. 20. Photograph of the PREX-1 pebble test facility. (Courtesy of University of California-Berkeley) 


\subsection{HEAT TRANSPORT SYSTEM}

\section{General Discussion on Liquid Salts}

Flibe is the primary coolant selected for the UCB baseline design. Extensive research and measurements on physical and chemical properties of liquid salts have been performed over the years. Also considerable operating experience from the MSRE exists that provides data and insight into fluoride salts as a heat transport medium. Flibe was used in both the primary and secondary loops of the MSRE and related test loops. Some physical properties such as thermal conductivity and infrared absorption are not yet known with the desired precision and merit additional measurements. Accurate measurements of the heat transfer parameters of fluoride salts at high temperature are challenging. Competing modes of heat transfer, i.e., conduction, convection, and radiation, must all be properly accounted for. Moreover, the heat transfer characteristics may change with time as the interface properties of the fluid-material boundary shift, particularly if scale form on the surface of materials.

The high optical transparency of liquid salts in the visible and near infrared promotes effective radiative heat transfer at FHR operating temperatures, while salt absorption in the far infrared can cause salts to act as participating media at these wavelengths. The current uncertainties in high temperature fluoride salt heat transfer parameters may impact the design requirements of individual heat transfer components by necessitating excessive conservatism in their designs. Part of the mission of FHR-TS component testing is thus to acquire improved fluoride salt heat transfer data using prototypic fluid boundary materials.

A critical issue in working with the flibe is beryllium control. Beryllium and its compounds are highly toxic and are designated as carcinogens. Therefore, all areas where beryllium access is possible will be contained within a Beryllium Control Area. While considerable experience has been gained in working with beryllium and successfully enforcing protection measures from the MSRE and other beryllium processes, beryllium control adds significant expense to any experiment. Thus developing a beryllium control program will need to be a primary consideration for any component testing employing beryllium-containing salts and in the design of the FHR-TS.

\subsubsection{Reactor Vessel and Primary Piping}

The FHR-TS vessel and primary piping will likely be a more complicated structure than was employed at the MSRE. A final design does not yet exist for either the vessel structure or piping. To a large extent the issues pertinent to the reactor vessel and primary piping are materials focused and thus mostly outside the scope of a component test-planning document.

The FHR-TS baseline design calls for a high temperature tolerant interior vessel. The vessel may be fabricated from a carbon-carbon composite, a high temperature structural alloy with a nickel-based cladding, or Hastelloy-N. The next layer in the vessel structure is most likely to be a poisoned buffer salt to provide flow into the vessel and to shut down the reactor if the primary vessel fails. Outside the buffer salt will most likely be a layer of salt tolerant insulation (likely a carbon foam). Adherence of the insulation layer to the exterior vessel is a key consideration as large, fully penetrating cracks in the insulation may allow the buffer salt to set up a convection loop and overheat the exterior vessel steel. Finally, the exterior vessel will be comprised of an ASME BPVC rated steel alloy. The FHR-TS exterior vessel baseline design calls it to be at a sufficiently low temperature during normal operation to freeze any fluoride salt that comes into contact with it as a result of insulation cracking, thus creating a frozen salt vessel liner. Tritium permeation resistance is also a design consideration for the intermediate heat exchanger to limit contamination of the secondary salt. 


\subsubsection{Liquid-Salt Pumps}

\section{General Discussion on Liquid-Salt Pumps}

The pumps included in this evaluation are mechanical, free-surface, centrifugal, vertical-shaft sump pumps. These pumps are generally divided into two groups:

1. Pumps with a long shaft with at least one shaft support bearing located in the pumped fluid.

2. Pumps with a short shaft with the impeller overhung.

The first group, long-shaft pump, generally includes reactor sodium pumps for various sodium fast reactors. A liquid-salt pump of this type was designed and operated at ORNL [25]. Long-shaft pumps have shaft support adjacent to the impeller provided by a journal bearing that is lubricated with the pumped fluid. This permits the use of long shafts to separate the drive motor and its sensitive electrical insulation and hydrocarbon lubricant from the intense radioactivity of salt-fueled reactors and high temperature.

The second group, short-shaft pump, includes the remainder of the liquid-metal and liquid-salt pumps that were developed for the MSR program. The MSRE pump is an example of short-shaft pumps. A brief description of the MSRE primary fuel pump is given in Sect. 2.5.4. The current baseline FHR-TS design uses a short shaft pump, located in the hot leg of the reactor primary circuit.

\section{Operating Experience}

A cross-sectional drawing of a long-shaft liquid-salt pump is shown in Fig. 21. The pump shaft was supported at the lower end with a liquid-salt-lubricated hydrodynamic journal bearing and at the upper end by an oil-lubricated ball bearing. Shaft seals were applied to the upper bearing region to prevent leakage of oil to the atmosphere or to the liquid salt.

The distinguishing features of the short-shaft pump are the short distance between the shaft support bearings and the overhung impeller. As a general characteristic, the MSR program pumps had the impeller and volute submerged in a vessel that served as an expansion tank in which there is liquid-free surface. The FHR-TS is expected to have a free surface in the pump expansion tank and in the reactor vessel, so these volumes must be connected by a low-flow-resistance channel to prevent pressure differences from causing differential level swell between the surfaces. A summary of the operating experience with liquid-salt and liquid-metal pumps during the MSR development program is given in Table 5.

Other than the shaft length, the primary difference between the short- and long-shaft pump is the means of supporting the shaft. The short shaft is usually supported with oil-lubricated ball bearings at two places located relatively close together. The long shaft is supported at its lower end with a pumped fluid-lubricated bearing and at its upper end with a conventionally lubricated bearing. The short-shaft design has the advantage of not requiring a salt-lubricated bearing. However, locating the shaft bearings significantly above the impeller results in the maximum impeller immersion depth being at most a few tens of millimeters [4] below the salt-free surface to control shaft flexing and vibration. 


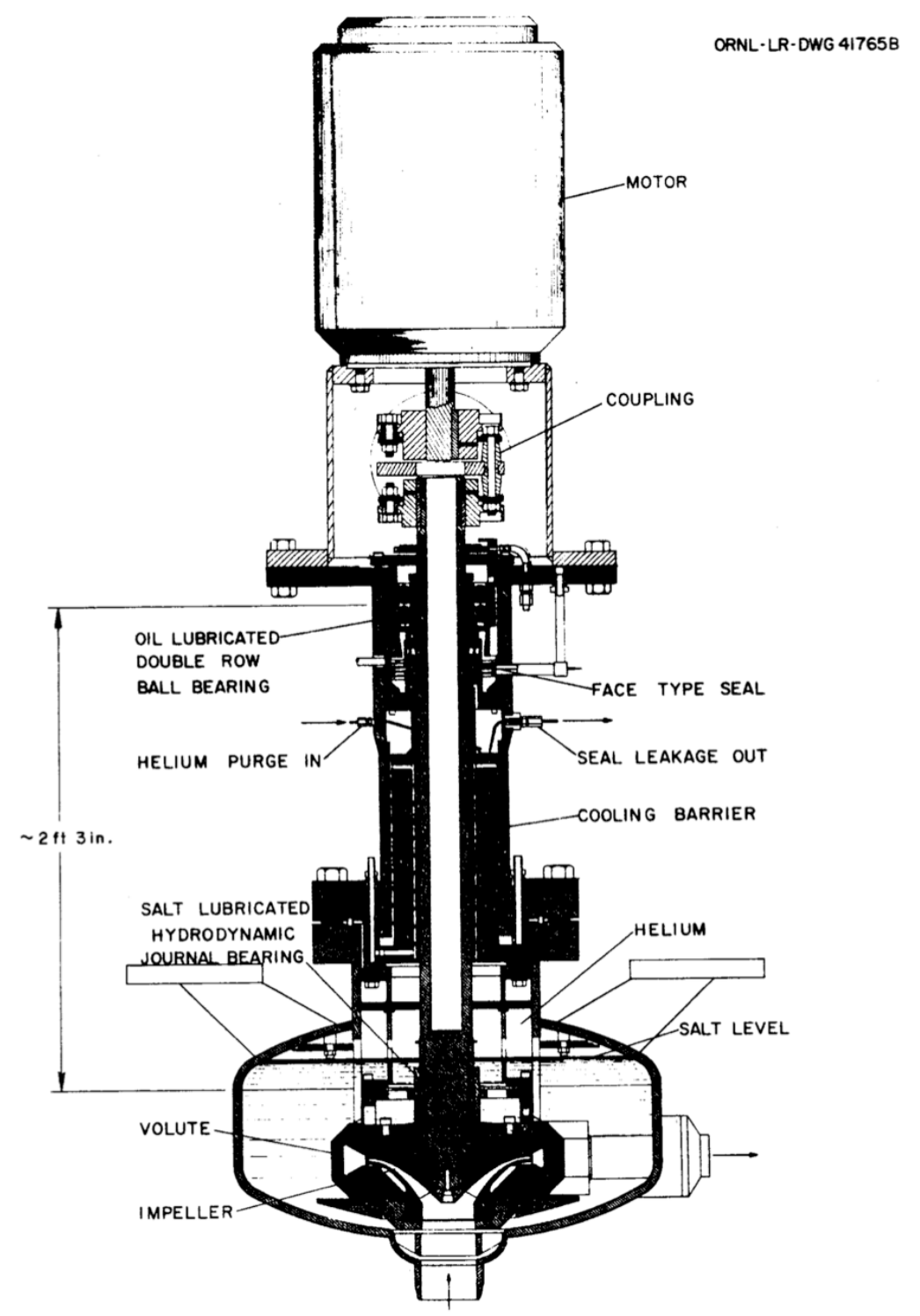

Fig. 21. Long-shaft liquid-salt pump with one liquid-salt lubricated bearing [7]. 
Table 5. Characteristics and operation time for ORNL salt and liquid metal pumps [9]

\begin{tabular}{|c|c|c|c|c|c|c|c|}
\hline Model & Fluid & $\begin{array}{c}\text { Head }^{\mathrm{a}} \\
\text { (ft) }\end{array}$ & $\begin{array}{l}\text { Flow }^{a} \\
(\text { gpm })\end{array}$ & $\begin{array}{l}\text { Speed }^{\mathrm{a}} \\
(\mathrm{rpm})\end{array}$ & $\begin{array}{c}\text { Temperature } \\
\left({ }^{\circ} \mathbf{F}\right)\end{array}$ & $\begin{array}{c}\text { Number } \\
\text { built }\end{array}$ & $\begin{array}{l}\text { Total } \\
\text { hours }\end{array}$ \\
\hline LFB & $\mathrm{Na}, \mathrm{NaK}$, and molten salt & 92 & 5 & 6000 & $1100-1400$ & 46 & $466,000^{b}$ \\
\hline DANA & $\mathrm{Na}, \mathrm{NaK}$, and molten salt & 300 & 150 & 3750 & $1000-1500$ & 10 & 57,000 \\
\hline $\mathrm{DAC}$ & Molten salt & 50 & 60 & 1450 & $1000-1400$ & 3 & 4,000 \\
\hline $\begin{array}{l}\text { In-Pile } \\
\text { Loop }\end{array}$ & Molten salt & 10 & 1 & 3000 & & 8 & $14,000^{\circ}$ \\
\hline MF & $\mathrm{NaK}$ and molten salt & 50 & 700 & 3000 & $1100-1500$ & 3 & $41,000^{\mathrm{d}}$ \\
\hline PKA & $\mathrm{NaK}$ and molten salt & 400 & 375 & 3550 & $700-1500$ & 2 & 21,500 \\
\hline PKP & $\mathrm{NaK}$ and molten salt & 380 & 1500 & 3500 & $700-1500$ & 4 & 45,000 \\
\hline \multirow[t]{2}{*}{$\begin{array}{l}\text { MSRE } \\
\text { fuel salt } \\
\text { pump }\end{array}$} & Molten salt & 50 & 1200 & 1175 & $1000-1225$ & $2^{\mathrm{e}}$ & 31,600 \\
\hline & Helium & & & & $100-1200$ & & 6,000 \\
\hline \multirow{2}{*}{$\begin{array}{l}\text { MSRE } \\
\text { fuel salt } \\
\text { pump }\end{array}$} & Molten salt & 78 & 800 & 1775 & $1000-1225$ & 2 & 24,600 \\
\hline & Helium & & & & $100-1200$ & & 4,000 \\
\hline $\begin{array}{l}\text { MSRE } \\
\text { Mark-2 } \\
\text { fuel salt } \\
\text { pump }\end{array}$ & Molten salt & 50 & 1200 & 1175 & $1000-1300$ & 1 & 14,000 \\
\hline \multirow[t]{2}{*}{ ALPHA } & Molten salt & 300 & 30 & 6500 & $850-1400$ & 1 & 6,000 \\
\hline & & & & & Total & 83 & 734,700 \\
\hline $\begin{array}{l}\text { At desig } \\
\text { Some pr } \\
\text { Includes } \\
\text { Include } \\
\text { Includes }\end{array}$ & $\begin{array}{l}\text { int. } \\
\text { were operated for periods o } \\
0 \mathrm{hr} \text { in-pile operation. } \\
\text { nuous operation of } 25,500 \mathrm{~h} \\
\text { ototype salt pump. }\end{array}$ & singl & 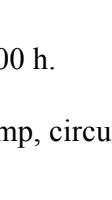 & $\mathrm{mol}$ & lt. & & \\
\hline
\end{tabular}

\section{Technology Issues and Potential Challenges}

Density and viscosity are the two physical properties of liquid salts that strongly affect pump design. Salt density mainly affects the torque requirements for the pump impeller and requires that the shaft has sufficient torsional strength and that the drive motor produces the required torque. Viscosity and materials compatibility of the liquid salt strongly affect the life characteristics of the hydrodynamic bearings, which are needed for long-shaft pumps.

The principal technology problems with large liquid-salt pumps were determined during the MSBE and MSBR programs [7]. These include

1. dynamic response of the rotating components assembly,

2. bearings,

3. thermal and radiation damage protection, 
4. shaft seals,

5. hydraulic design, and

6. fabrication and assembly.

Of particular importance for the extent of this document are potential problems with bearings, seals, and fabrication and assembly issues. Sophisticated CAD tools, FEM solvers, and dynamic analysis software available today allow for predicting the system response of complex systems more realistically than what was available during the conceptual design of the MSBE. However, long-term response as well as response to temperature cycling of rotating elements requires experimental scrutiny.

The short-shaft pump does not require development of bearings, and its reliability is enhanced by the use of conventionally lubricated ball bearings that have good long-term life based on large statistical samplings. The long-shaft pump configuration requires a liquid-salt-lubricated journal bearing near the lower end of the shaft. The problems identified for long-shaft pumps in the MSBR program [7] include

1. the design of the bearing to produce the hydrodynamic lubricating film with liquid salt,

2. the selection of the bearing materials and their form (hard surface coating or integral body configuration),

3. the accommodation of differential thermal expansion if integral body construction is used, and

4. the design of a bearing mounting arrangement that will accommodate some thermal distortion between the pump shaft and casing without interfering with the lubricating film in the bearing.

Limited work was performed to develop salt-lubricated bearings that would permit the use of longshaft pumps. Recent work for nitrate salt pumps has shown the practicality of long-shaft designs, but additional work will be required to develop and qualify bearing materials for use with fluoride salts [26].

Bearing technology has made significant advancements since the design and operation of the MSRE. New materials and fabrication techniques that were not available or were not adequately mature have become available with improvements in science and technology. Diamond and other advanced ceramic composite bearings in particular appear to be a promising technology that was not available during the prior development campaign. Ceramics such as high-purity silicon carbide offer high strength as well as high abrasion and wear resistance, making it an excellent material of choice for bearing. Both high-purity silicon carbide and diamond are also known to be corrosion resistant in a liquid-salt environment.

Another bearing technology that can be employed in the liquid-salt environment is magnetic bearings. Both passive and active magnetic bearing may be of use in a liquid-salt environment. Active magnetic bearings are commonly used by industry for rotor levitation. The challenge of active magnetic bearings is that they require sophisticated control hardware, such as digital signal processors, amplifiers, digital-toanalog converters, analog-to-digital converters, and control software. Furthermore, they require transducers to detect the shaft location to provide input for feedback control. On the other hand, passive magnetic bearings do not require any control system. However, they have the disadvantage of lower stiffness and lower damping than similarly sized active counterparts. A remaining materials issue with passive magnetic bearings near liquid salts is ensuring that the permanent magnets remain sufficiently cool to avoid demagnetization.

A shaft seal is required with either the long- or short-shaft pump to maintain separation between the lubricant in the upper shaft bearing and the cover gas in the pump tank. The following issues are anticipated [7]: 
1. the fabrication of a bellows to accommodate the required pump shaft diameter,

2. the attachment of the rotor wear surface with respect to the axis of shaft rotation,

3. the attachment of the stator wear element to the bellows to obtain a hermetic joint,

4. the assembly of the stator element to provide both squareness and concentricity of its wear surface with the axis of shaft rotation to close tolerances.

Shaft seals proportioned for 75-mm-diam shafts have been operated continuously for more than $25,000 \mathrm{~h}$. However, proof-test seals should be developed programmatically for larger diameter pump shafts for commercial-scale FHRs.

\section{Other Considerations}

The pressure-containing parts of the MSRE pump were designed in accordance with Section VIII of the ASME Boiler and Pressure Vessel Code and Code Interpretation Cases 1270N and 1273N for primary vessels. The primary pump for the FHR-TS can be designed and fabricated in a similar fashion with appropriate Code Cases.

Contact-type seals were used above and below the bearings to confine oil to the lubrication system. Oil leakage into the salt could result in crud accumulation and primary coolant flow path blockage, thus seal leakage is a significant design issue. The seals used for the MSRE primary pump had stationary graphite-based rings supported on flexible stainless steel bellows. The seals in the seal assemblies of the MSRE pump were of the balanced-piston type and were designed to accommodate the pressure transients on the lubricant side that were 14 to $55 \mathrm{kPa}$ higher than that in the pump bowl. Under these conditions, the lower seal accepted, without opening, pressure transients in the pump bowl as high as $450 \mathrm{kPa}$ or as low as $7 \mathrm{kPa}$. Labyrinth-type seals were used on the impeller inlet and support shrouds. These seals were O-rings fabricated of Hastelloy-N [6].

Sealing techniques and sealing materials have also made significant progress over the past few decades, and the pump bearing seal design will need to be revisited with an eye towards modern design capabilities to obtain an optimized pump design [27]. In particular, dry gas type rotating seals have become widely available over the intervening decades. These systems are widely used in the turbomachinery, chemical, oil and gas, etc. industries and can operate at elevated temperatures. However, temperature limits are typically below FHR operating temperatures, so cooling and additional development will be required for FHR-TS application.

Since the primary pumps are located on the hot legs, they may be subject to high temperature excursions under certain transients, such as Anticipated Transient Without Scram (ATWS) conditions. Therefore, portions of the pumps that are in direct contact with the coolant must be fabricated of material that can perform well at high temperatures and withstand thermal cycles. The pump sump and the pump blades are examples.

In general, the vapor pressure of $\mathrm{BeF}_{2}$ in the cover gas of the pump tank will be very low, but the potential for condensation of $\mathrm{BeF}_{2}$ (which is the most volatile component of flibe and has a freezing temperature of $552^{\circ} \mathrm{C}$ ) should be considered in the design of the pump tank and gas purge system. 


\subsubsection{Intermediate Heat Exchanger}

\section{General Discussion on IHX}

The Intermediate Heat Exchanger (IHXs) in the FHR-TS serves as the interface structure between the Primary Heat Transport System (PHTS) and the Intermediate Heat Transport System (IHTS) transferring heat from the primary to the secondary coolant. The IHX is a salt-to-salt heat exchanger with the primary

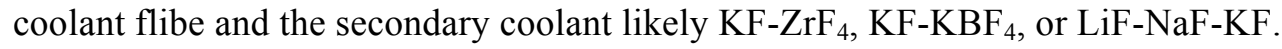

No final design decision has been made for the IHX. Potential design choices include shell and tube, plate/fin, and micro-channel type. Independent of the IHX type, the design must address all aspects of manufacturing and operation including materials, design, fabrication, examination, testing, and in-service inspections that are used in the construction and operation of the respective heat exchanger pressure boundaries and internal designs.

As noted in Sect. 2.3.3, the FHR IHX will be required to reject roughly twice the amount of heat as the MSRE heat exchanger, with a smaller log-mean temperature difference. One potential and near-term design option would be to use a larger shell-and-tube design similar to that of the MSRE, but configured for single-pass flow. A photograph of the primary heat exchanger subassemblies of the MSRE is shown in Fig. 22.

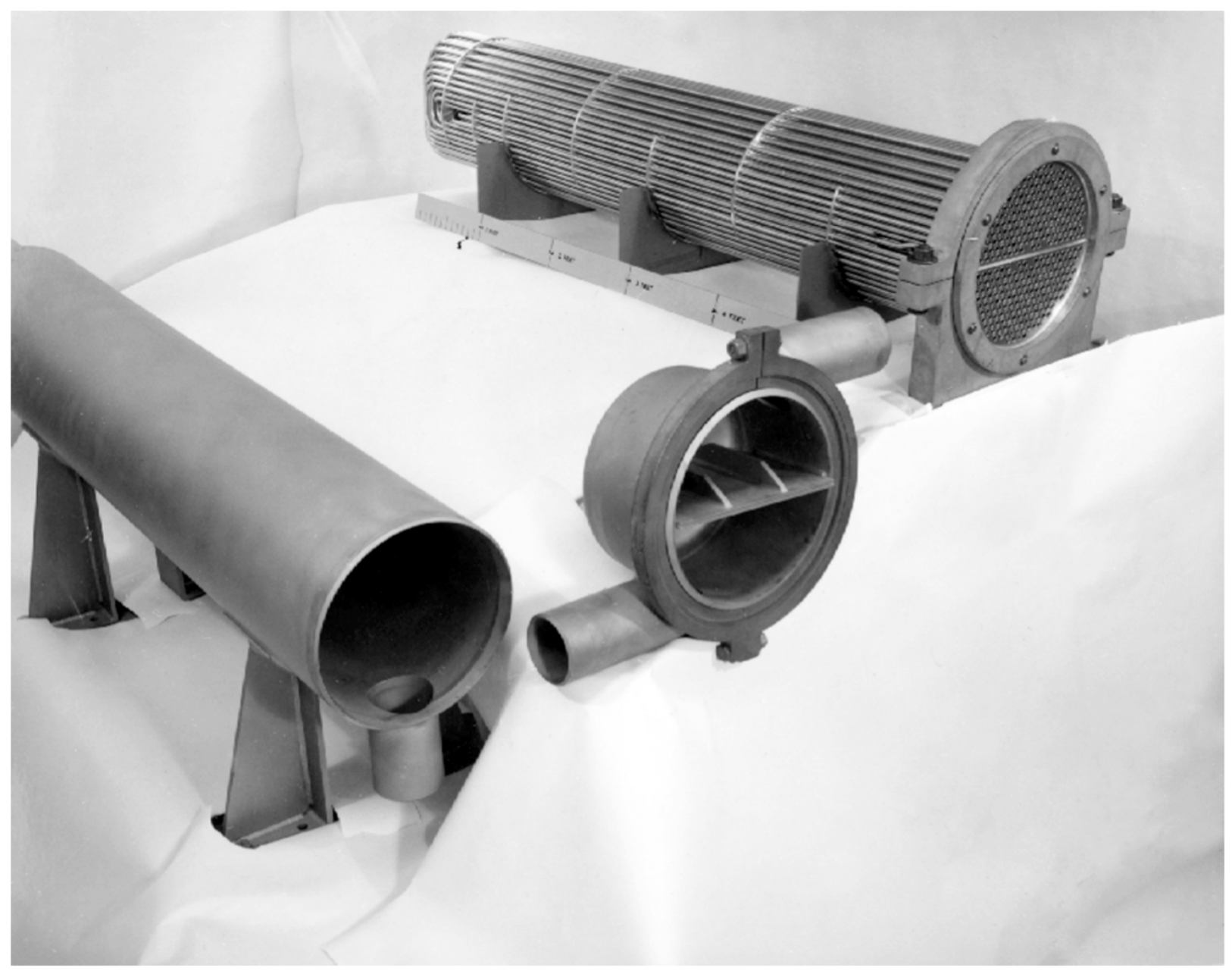

Fig. 22. Primary heat exchanger subassemblies of the MSRE. 
A comparison of major operating parameters for the MSRE and the FHR-TS is given in Table 6. These numbers are given as the results of preliminary calculations for comparison. They are not intended as design parameters for the IHX.

Table 6. Comparison of major operating parameters between the MSRE primary heat exchanger and the FHR-TS baseline design Intermediate Heat Exchanger

\begin{tabular}{|c|c|c|}
\hline & MSRE & FHR-TS* \\
\hline Heat Load (MW) & $10(7.4 \mathrm{MW}$ actual $)$ & 16 \\
\hline Primary Coolant & $\mathrm{LiF}+\mathrm{BeF}_{2}+\mathrm{ZrF}_{4}+\mathrm{ThF}_{4}+\mathrm{UF}_{4}$ & $\mathrm{LiF}+\mathrm{BeF}_{2}$ (flibe) \\
\hline Side & Shell-side fluid & Shell-side fluid \\
\hline Flow Rate $(\mathrm{kg} / \mathrm{s})$ & 150 & 70 \\
\hline Inlet Temperature $\left({ }^{\circ} \mathrm{C}\right)$ & 665 & 704 \\
\hline Outlet Temperature $\left({ }^{\circ} \mathrm{C}\right)$ & 635 & 600 \\
\hline Secondary Coolant & $\mathrm{LiF}+\mathrm{BeF}_{2}$ (flibe) & $\mathrm{KF}-\mathrm{ZrF}_{4}$ \\
\hline Side & Tube-side fluid & Tube-side fluid \\
\hline Flow Rate $(\mathrm{kg} / \mathrm{s})$ & 105 & 64 \\
\hline Inlet Temperature $\left({ }^{\circ} \mathrm{C}\right)$ & 550 & 545 \\
\hline Outlet Temperature $\left({ }^{\circ} \mathrm{C}\right)$ & 595 & 690 \\
\hline Effective Log Mean Temperature Difference $\left({ }^{\circ} \mathrm{C}\right)$ & 56 & 50 \\
\hline
\end{tabular}

* FHR values are scaled from the UCB PB-AHTR design [18].

\section{Requirements for the Intermediate Heat Exchanger}

The primary functional requirements of the IHX are given in Sect. 2.3.3. Additionally, the IHXs are expected to maintain their structural integrity during their design lifetime that includes thermal transients caused by normal operations, various plant upsets, and emergency operating conditions. The much lower LMTD of the FHR-TS IHX requires that it operate in single-pass, counter-current flow. In the case of shell and U-tube type heat exchangers, this requires baffling on the shell side to generate counter-current flow, differential expansions between tubes and shell must be accommodated without the use of bellows, and thermal stresses at critical locations, such as tube sheets and nozzles, should be minimized.

The IHX design must be able to withstand thermal cycling due to cooldown/heatup cycles that will occur during scheduled shutdown. Although the thermal stress loading on the component will be controlled by multiple means such as low rate of change in reactor power as well as external heat input to the component to maintain a quasi-homogeneous temperature profile, stresses caused by differential expansion could be the dominant life-limiting factors, and therefore must be accommodated by the IHX. Additionally, stresses that occur within the IHX due to significant local thermal gradients must be analyzed and design solutions identified. Flow-induced vibration and wear can become issues in untested heat exchanger designs and must be eliminated early in the process. The MSBR IHX was designed to use knurled tubes to enhance heat transfer due to the relatively low thermal conductivity of fluoride salts, and the potential for enhancement of surfaces in the FHR-TS IHX must also be considered.

The IHX is expected to have at least a 60 -year lifetime and be replaceable. The IHX design should also take into account possible maintenance needs over the anticipated life of the component. The IHX designs must also incorporate the ability to perform appropriate inspection and maintenance activities. 


\section{Operating Experience}

Conventional heat exchanger designs (shell and tube, plate type, etc.) are ubiquitous in a variety of industries, and many of the same design practices for those heat exchangers (thermal design, structural analysis, etc.) are directly applicable to molten salt systems. However high temperature molten salt systems also introduce some unique considerations not typically considered. These include the use of unconventional materials for heat exchanger construction, joining issues of those materials, unique corrosion considerations (driving materials selection and operation processes), potential freeze/thaw cycles, and the high temperatures $\left(>700^{\circ} \mathrm{C}\right)$ expected in designs that use these salts. Significant experience, however, under these conditions can be used to guide the IHX design.

The MSRE primary heat exchanger operated for $22,000 \mathrm{~h}$ in the salt circulation mode and $13,000 \mathrm{~h}$ in the equivalent full-power mode. During this period, the overall heat transfer coefficient of the heat exchanger did not change, indicating that there was no buildup of scale and no evidence of gas filming. Postexperiment investigations of the unit confirmed this hypothesis.

Liquid-salt systems and liquid-metal systems share many of the design- and operation-related problems. Therefore, design, fabrication, and operating experience gained in the liquid-metal systems is directly applicable to liquid-salt systems, and will be very useful from the concept to manufacturing phases. Development of high-temperature design criteria, thermal stress analysis of complex geometries, and the experience in the development of techniques for the fabrication of thick tube sheets and joining of tubes to tube sheets are a few examples that the Liquid-Metal Fast Breeder Reactor (LMFBR) experience will be useful although the base materials are not the same.

\section{Technology Issues and Potential Challenges}

For conventional heat exchanger designs, fabrication and assembly issues are limited to those specific to the materials being used (e.g., work hardening of Hastelloy-N, and joining processes). Specific joint designs, fabrication procedures, and inspection procedures would not present critical challenges if the MSRE heat exchanger design is copied. Heat exchanger fabrication issues were both successfully dealt with during the MSRE in preliminary high-temperature tests and proven during the long-term operation of the MSRE heat exchanger during reactor operation. Figure 23 shows the tube to tube-sheet joint in the MSRE primary heat exchanger. The construction material of both tubes and tube sheet was Hasteloy-N (at that time called INOR-8), and the joint was fabricated using a weld at the tube end (which was first expanded into the tube sheet, and a backup braze at the opposite side of the tube sheet. The braze material was a gold-nickel alloy and was introduced into the tube to tube-sheet interface through the feeder hole shown in the drawing. This particular configuration in the MSRE performed well over 22,000 $\mathrm{h}$ of fuel circulation without any leaks.

Bi-clad and tri-clad heat exchanger tubing that could incorporate a corrosion-resistant cladding material with a high temperature base alloy material may also be used as an alternate material selection to Hastelloy-N. The use of more advanced structural materials (higher strength) or alternate construction techniques would require additional development effort. For instance, refractory metal-based alloys are challenging to machine because of brittleness at low temperatures, and are complicated to weld due to high oxidation rates at the interface. However, industrial design and fabrication methods are more advanced than those available during the construction of the MSRE primary heat exchanger and should improve confidence that successful heat exchanger designs can be developed. 


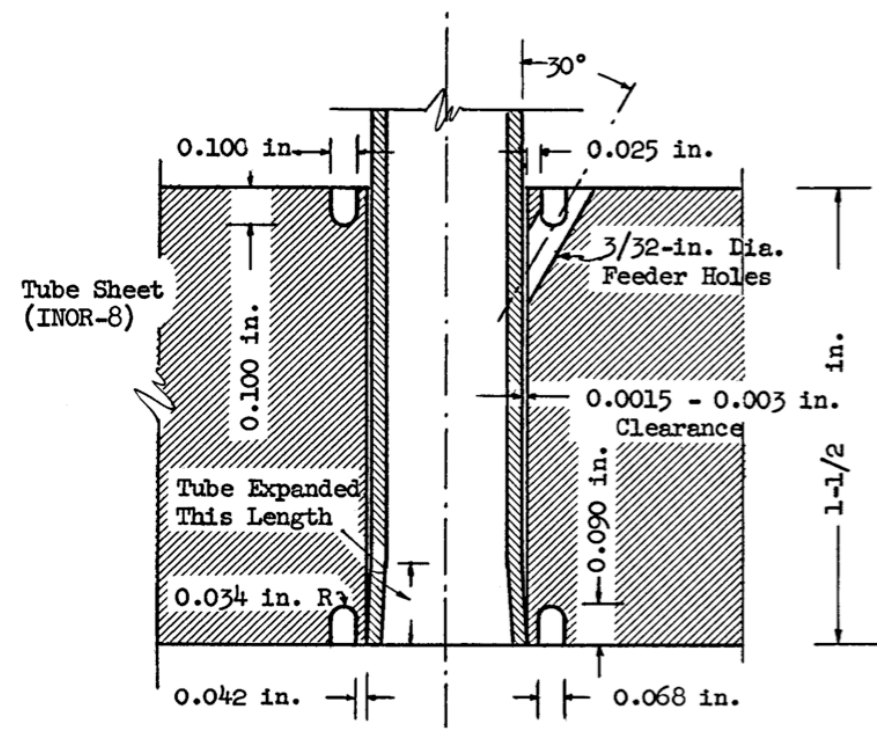

Fig. 23. Tube-to-tube sheet joint in the MSRE primary heat exchanger.

High salt melt temperatures introduce additional operational constraints. In the event of a prolonged loss of heat generation in the reactor, the coolant temperature in the PHTS as well as the IHTS may fall critically over time, potentially leading to freezing of the salt. As long as salt remains in the heat exchanger, a means must be assured to maintain temperatures above the melt temperature. Auxiliary systems that might use electrical heating or high temperature gas heating can be used to provide guard heating in this event to prevent the salt from freezing. For example, the MSRE primary heat exchanger employed 30-kW electrical resistance heaters installed outside the shell to avoid freezing and provide the capability to keep the heat exchanger above the salt melt temperature during salt fill processes.

\section{Emerging Technologies}

New heat exchanger technologies may be available for use in the IHX application, including Printed Circuit Heat Exchangers, Formed Plate Heat Exchangers (FPHE), and Hybrid Heat Exchangers ( $\left.\mathrm{H}^{2} \mathrm{X}\right)$. These heat exchangers are formed from alternating layers, typically hot-cold-hot. These heat exchanger designs offer the possibility of a smaller footprint and potentially lower capital cost. However, no experience exists in designing, constructing, and operating these systems using high temperature molten salts, and it would be expected that significant development of these designs would be needed before they could be incorporated into the FHR design.

\subsubsection{Secondary Heat Exchanger}

The secondary heat exchanger in the FHR-TS interfaces the intermediate salt-cooled heat transfer loop to the Brayton cycle power conversion system. Liquid-to-gas heat exchangers are very important commercially; however, they commonly exchange heat between water and air. Typical applications include automotive radiators, oil coolers, refrigeration equipment, intercoolers, etc., and a significant amount of design and operating experience exists for these systems. This type of heat exchanger is also a component in one version of the NGNP high temperature gas reactor design, serving to separate the primary helium reactor coolant system from an intermediate molten salt coolant loop. As development of this technology is undertaken for the NGNP program, it can be directly applied to the AHTR test reactor. 
A molten salt-to-gas heat exchanger design study was performed for the MSR program in the late 1950s [28]. A cross-sectional drawing of the preconceptual design for the heat exchanger is shown in Fig. 24. The heat exchanger geometry employed serpentine salt tubes with the gas flowing straight through the heat transfer matrix. A four-pass arrangement was provided to allow for differential thermal expansion in the tubing. More recently the NGNP program has investigated helium-to-liquid salt heat exchanger design issues [19, 29].

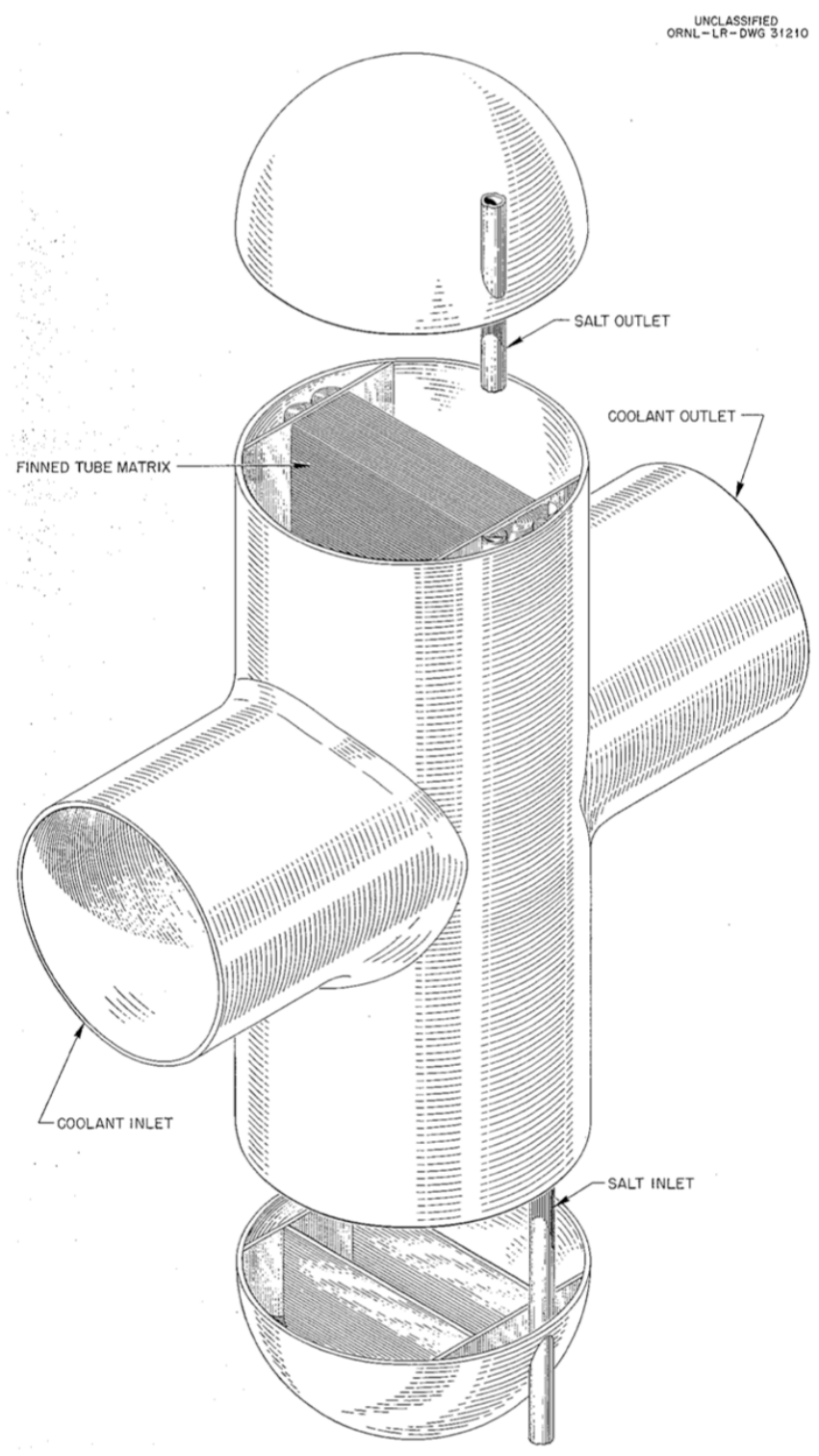

Fig. 24. Early salt-to-gas heat exchanger design.

As in conventional heat exchanger design, the salt-to-gas heat exchanger design must include considerations such as thermal stress analysis, pressure loading, heat transfer analysis, and identification of fluid/structure interactions. Although physical properties of both fluids are reasonably well known (with the possible exception of the absorption characteristics of the salt and its impact on radiative heat transfer, as noted earlier), these designs will be specialized in that pressure differentials between the primary and secondary systems are large, and it will be critical for the design to minimize temperature differentials between the molten salt and gas as well as the pressure drop on the gas side in order to 
maintain high efficiencies in the power conversion system. Additionally, because the gas has a low thermal conductivity, the gas velocities will have to be high or the heat transfer increased on the gas side by using enhanced surfaces or large surface areas, or both, in order to decrease the heat exchanger size and improve efficiencies.

Because the gas flow involves a very large volumetric flow rate, it is desirable to design the gas side with a simple flow path providing low pressure drop to minimize the circulating power, which is the product of the volumetric flow rate and pressure drop. Because salt has very high volumetric heat capacity and a very small volumetric flow rate, the salt side can be designed to have a high pressure drop without significant concern over pumping power. Because the secondary heat exchanger operates with a relatively small LMTD, it is important that the design provides for effective counter-flow heat exchange and that it effectively distribute the flow to different portions of the heat exchanger to provide high heat exchange effectiveness.

Since this type of heat exchanger has not been previously developed, they present several specialized design issues, some of which have been discussed in previous sections. Materials of construction will have to be compatible with both the intermediate salt and the gas. Salt chemistry will have to be maintained and the impurity levels in the gas stream controlled to prevent excessive material corrosion. Potential materials of construction include Hastelloy-N, or a higher strength alloy clad with a nickelbased alloy. Longer-term designs might include ceramics or composites as the structural material.

Additional specialized issues for salt-to-gas heat exchangers may include design features that allow draining of the salt and/or accommodation of freezing (or local freezing after draining) when the system cools. The phase change issues may be particularly problematic if the design incorporates very small cooling channels (such as might be present in circuit board-type heat exchanger designs). Long-term materials transport within the salt of the intermediate loop must also be considered both because of material degradation and because the salt-to-gas heat exchanger will be the coldest portion of the loop and will tend to be where dissolved materials tend to deposit.

\subsubsection{Salt Processing and Inventory Control System}

Few of the details of the salt cleaning systems have yet been designed, thus the system maturity remains mostly to be determined. However, the dominant system maturity issues most likely will be with the transfer and handling of the salt rather than in its processing which was demonstrated with the MSRE. Chemical processing of the MSRE flush and coolant salts was reported on as ORNL-TM-2578 [30]. Further, the instrumentation used to assess the MSRE salt was described in ORNL-TM-1853 [14].

The MSRE used a flush salt to remove impurities in the primary loop before fuel salt was added. The FHR-TS is likely to use coolant salt recovered from the MSRE as its primary salt, to limit the need to produce additional enriched lithium-7. Because the inventory of the MSRE coolant salt is limited, a second reactor volume of flush salt is unlikely to be available, and thus on-line chemistry control to remove oxygen and other contaminants will be required.

Issues such as transfer pump seals, instrumentation, and sampling valves will require significant development before the FHR PICS development is completed. A primary source for salt impurities is corrosion of the contacting materials as well as air and moisture ingress. Thus the scale and nature of chemical control necessary will be largely determined by the salt contacting materials selected. The principal process for impurity control within the primary salt will be by fluorination with impurities being either converted to fluoride precipitates or gaseous species that can be physically removed from the primary coolant. How and whether to remove graphite fines from the primary coolant salt has not yet been determined. 


\subsubsection{Cover Gas Chemistry and Pressure Control System}

In general, gas pressure measurement and control are well understood and fully commercialized. The key issues for an FHR's cover gas control system are tritium and moisture removal from the gas. Moisture removal from an argon gas stream is well known technology and can be accomplished by either physical or chemical approaches. Apart from tritium removal, the cover gas cleaning system will be developed using commercial technologies as part of the plant design process.

Tritium is generated by neutron capture reactions within the primary coolant salt. Tritium emerges from the salt both due to inherent diffusion through the structural materials and by sparging the salt with a carrier gas. Alternatively, tritium can be removed from the primary salt by application of intense ultrasonic energy. Ultrasonic degassing is industrially applied both to light alloy casting and glass melts but has not been applied to fluoride salts [31]. Removing the tritium from the primary salt is important for limiting its ingress into the secondary coolant salts and thereby increasing the difficulties of secondary heat transfer system access. Tritium may also be removed from the primary system cover gas and the power conversion system gas. Further, tritium and its decay product $\left({ }^{3} \mathrm{He}\right)$ are now valuable commodities to be captured and sold.

The current leading candidate structural materials for FHRs are clad structures. The particular layering structure has not yet been determined, thus the details of tritium permeation through structural materials in the FHR remains to be determined. Clad materials have the potential to greatly reduce tritium diffusion by including diffusion barriers within their layering structure. Tritium diffusion is one of the design parameters being considered as the structural material design progresses. Issues associated with tritium diffusion through structural materials in molten salt reactors were studied and reported on during the prior molten salt reactor development efforts [20].

While the specific nature of the need for tritium trapping at the FHR-TS remains unresolved due to design immaturity, the details of the technical maturity for the tritium control system cannot be fully evaluated. Tritium traps continue to be developed and studied in support of fusion energy. In particular, transfer for tritium from neutron irradiated lithium blankets to yttrium metal traps via argon gas has recently been demonstrated [32]. More generally, hydrogen traps continue to be developed in support of fuel cell vehicles.

\subsubsection{Valves for Liquid Salts}

\section{Mechanical Valves}

Early fluoride salt reactor concepts were developed in parallel with liquid-metal coolant systems, especially in the case of the aircraft reactor work in which the fuel was carried in a fluoride salt but the main heat transfer was accomplished with sodium. Many of the key components, including pumps, used in the salt and liquid-metal circuits encountered similar design requirements. Often, solutions to needs of high temperature liquid-metal systems also served well in fluoride salt applications. In the case of mechanical valves, however, more contrast developed between valves for high temperature liquid-metal service and valves for high temperature fluoride salt service.

Two primary types of valves are commonly used in piping systems. Throttling valves are designed to provide a variable flow resistance. Shut-off valves are designed to be capable of providing low leakage when closed. In practice, these functions are commonly performed by different valve designs. When a throttling valve is not designed for shut-off service, its design can be optimized to provide a nearly linear change in flow resistance as the control actuator is moved. Conversely, for shut-off service it is necessary to form a tight closure between the movable element in the valve and a valve seat. Because molten salts 
are excellent fluxing agents, metal-to-metal contacts are subject to galling and self-welding, so the identification of suitable combinations of materials creates a challenge. The same holds true for sliding surfaces in the valve. Commonly, different materials must be used for different parts of the valve, and mismatching between the thermal expansion coefficients must be accommodated by the design. Finally, some mechanism must be provided to apply force to the movable element in the valve in order to open and close it, unless the valve is a simple check valve where the fluid flow provides the activating force.

Mechanical valves have been developed and successfully demonstrated for liquid-metal systems. One approach used in actuator shaft seals for valves in high temperature sodium service is the use of frozen sodium to complete the seal in the actuator shaft. Static seals using frozen liquid salts have been used with freeze flanges, access ports, and freeze valves. However, the glass-like character of the frozen salt complicates its use in a dynamic seal, and frozen seal valve shafts have not been applied to fluoride salt systems. Other alternatives may be considered, including bellows seals that can contain molten salt or actuators that do not require mechanical penetrations, such as magnetically actuated valves.

Limited experience exists for mechanical valves in fluoride salt service. One of the pump test stands used in the development of pumps for the MSRE included a mechanical throttling valve. This loop was originally constructed during development and testing of Aircraft Reactor Test components; it may have been intended for liquid metal service. As described in ORNL/TM-3344 [33], the loop was used to test coolant pumps in a sodium fluoroborate salt circuit. Salt flow could be varied between 400 and 1000 gpm by adjustment of the throttle valve. A metal bellows was used for the steam seal, and an automatic gas pressurization system was connected to a chamber surrounding the bellows to ensure that the difference in pressure across the bellows was kept within acceptable limits. The loop used freeze valves for isolation of the drain system. Later loops constructed for the MSRE project used variable speed pump motors to control flow, avoiding the need for mechanical throttling valves.

An assessment of mechanical valves in molten salt service was presented in ORNL/TM-1855 [5]. A program to develop a fast-acting shutoff valve for the Aircraft Reactor Test is described. This valve consisted of a spherical metal plug that fitted into a similarly shaped metal seat. Positioning of the plug was accomplished by the movement of a stream through a bellows seal. Guiding was on the helium side of the bellows. Valve sticking was observed; the indication was that galling on the exterior guides may have been the cause. The development effort ceased before a clear resolution to the concerns was obtained.

\section{Freeze Valves}

Considerable experience was gained in the use of freeze valves in the MSRE reactor, the earlier aircraft reactor program, and a host of associated test loops. This technology continues to be used in test loops today. The basic technology for use of freeze valves as slow-acting isolation valves is well-known. Specific details, including lifetime under repeated cycling, are application specific.

Further development in freeze valve technology could be directed toward faster operating speeds. In all cases, the thermal and mechanical stresses associated with repeated freeze and thaw operations must be addressed in the design and material selection for freeze valves. The one salt piping leak at the MSRE, occurring as the final shutdown sequence was being performed, was encountered at a freeze valve. A cooling shroud was modified in the field to make it stiffer; this modification resulted in additional mechanical stress at a key location in the freeze valve salt piping and its eventual fatigue failure. 


\subsection{DIRECT REACTOR AUXILIARY COOLING SYSTEM}

The DRACS concept was originally developed for the Experimental Breeder Reactor-II (EBR-II), and has been refined and improved in later liquid-metal reactor designs, such as the Sodium Advanced Fast Reactor (SAFR) and the European Pressurized Reactor (EFR) [34]. In these reactor designs, natural circulation auxiliary decay heat removal systems were designed to be always in operation.

Natural circulation driven decay heat removal systems have a substantial experimental pedigree. Both EBR-II and Super-Phenix included passive decay heat removal loops. The primary differences between the FHR DRACS implementation and those of earlier systems are (1) external event hardening of the exposed external heat transfer components and (2) the use of a fluidic diode in the primary salt loop to limit parasitic heat loss during normal forced-circulation operations.

\section{Operating Experience}

EBR-II included a natural circulation-driven shutdown cooler system. The in-vessel portion of the shutdown cooling system was an immersion-type bayonet heat exchanger, as shown in Fig. 25 [35]. The heat exchanger consisted of two concentric pipes approximately $8 \mathrm{~m}$ long, the outer pipe being closed at the bottom. The inner pipe is thermally insulated from the outer to provide a greater thermal head for natural convection. The shutdown cooler was inserted into a vertical thimble immersed in the primary tank, with a sodium thermal bond filling the gap between the thimble and the heat exchanger. The EBR-II shutdown cooler system used $\mathrm{NaK}$ as the coolant.

The coolant enters the top of the inner pipe of the bayonet cooler and flows downward where the flow reverses direction as it enters the annular region between the outer and inner pipes. As the coolant rises up the outer pipe, it gains heat through conduction from the pool sodium. Upon exiting the bayonet heat exchanger, the coolant flows up into a finned-tube air heat exchanger. This liquid metal-to-air heat exchanger is located in a chimney outside the reactor containment building. Air flow into the chimney is controlled by dampers. Heat is transferred from the coolant to the atmosphere by natural convection. The heat transfer circuit is completed as the cooled coolant flows downward into the inlet of the bayonet heat exchanger.

The rate of heat release from the system is controlled by the position of the stack dampers. Normally the dampers are actuated by automatic control; however, manual control was also provided. During reactor operation, the dampers are normally closed to limit parasitic heat loss. 


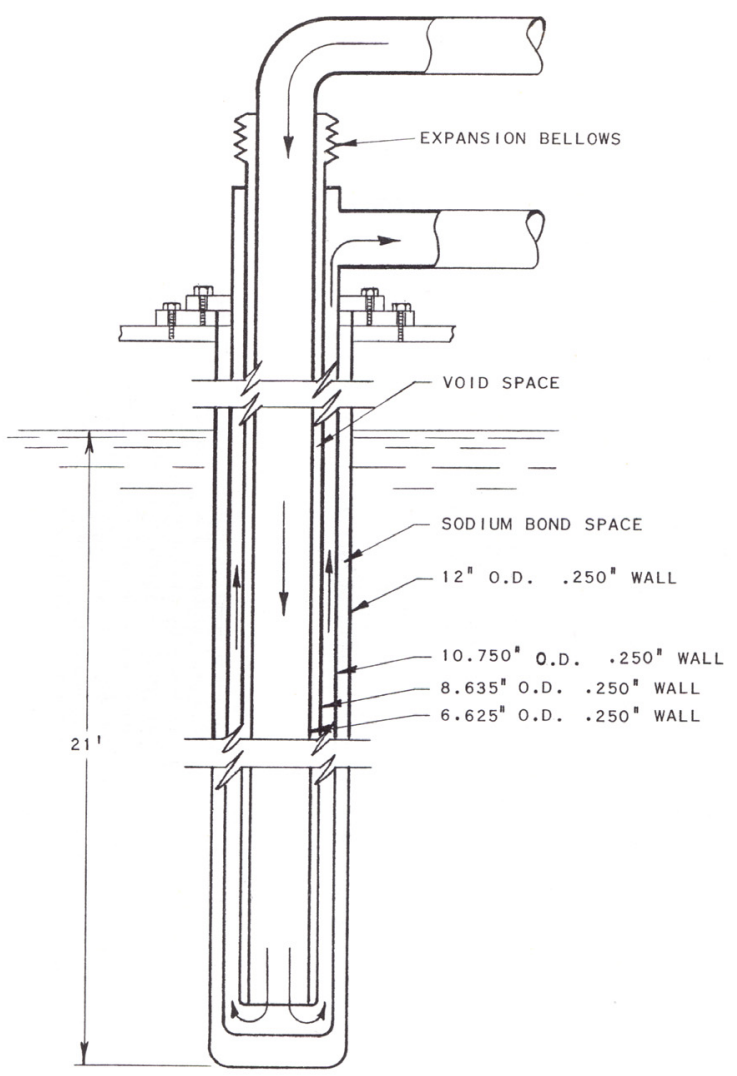

Fig. 25. Shutdown cooler bayonet heat exchanger in EBR-II [35].

The performance of the passive shutdown cooling system was demonstrated at EBR-II with a series of loss-of-heat sink tests [36]. The shutdown cooling system demonstrations involved stopping the reactor primary cooling and observing the resultant system transient. The tests successfully demonstrated that the shutdown cooling system functioned as predicted and provided adequate heat removal to prevent core damage.

The SAFR reactor design also included a DRACS which was evaluated by the NRC in a preliminary safety evaluation report [37]. The SAFR DRACS, however, was not designed as a safety component. The major issues in making the DRACS a safety-related system were developing a safety-grade turn-on mechanism for the in-vessel cooling loop and impact (tornado) hardening the external sodium-to-air heat exchanger. The SAFR DRACS employed helium-driven valves to prevent the sodium loop from flowing during normal operations. Also, the dampers on the air loop were intended to be kept closed during normal operations to prevent sodium freeze-up as well as to limit parasitic heat loss.

The Super-Phenix LMFBR also included a DRACS that was reviewed and approved by French safety regulators [34].

\section{Current Technology Status}

The DRACS is the primary emergency decay heat removal system for the FHR-TS. While at test reactor scale a dedicated decay heat removal system is not likely to be necessary to avoid fuel damage, a primary purpose for the FHR-TS is to demonstrate the functionality of the safety systems for larger FHR plants. 
One of the concerns with the SAFR DRACS design was the possibility of making the in-vessel cooling loop initiation system safety-grade. The FHR-TS design replaces active valves of the SAFR with a passive fluidic diode with no moving parts. The fluidic diode design thus needs to be demonstrated and approved as a safety-related nuclear component. The other SAFR DRACS identified design issue was external event hardening of the exposed components. External event hardening of exposed components is a mature discipline.

The FHR-TS DHX will most likely use a shell-and-tube heat exchanger design. Shell-and-tube heat exchangers have a long operating experience across a wide range of temperatures and chemical constituents. The FHR-TS NHX will most likely have tube banks with salt flowing on the tube side, and air flowing in cross flow over two to five rows of tubes so that the flow resistance for the air will be low. The coolant for the FHR-TS DRACS will have a lower melting temperature than the primary coolant to reduce the possibility of DRACS freeze-up. Also, the DHX will be designed to have substantially lower heat transfer resistance than the NHX, so that the temperature drop across the DHX will be deterministically substantially smaller than the temperature drop across the NHX. The fluidic diode in the in-vessel loop will only partially block primary coolant flow. The residual primary coolant flow will be used to keep the DRACS coolant liquid during normal operations. However, the DRACS loop will require trace heating for start-up and long-term shutdown.

A primary safety advantage of FHRs is the lack of mechanisms for pressurizing primary containment. To preserve this advantage, the DRACS primary coolant will not be a liquid metal with its stored chemical energy potential. A liquid fluoride salt DRACS cooling loop has not previously been demonstrated, so designing and demonstrating this system will be a primary purpose for FHR component and system testing. An additional consideration for the DRACS coolant is the possibility of tube leakage between the DRACS salt and the primary coolant salt. The DRACS coolant salt should thus be chemically separable from the FHR primary coolant (i.e., not contain ${ }^{6} \mathrm{Li}$ ) to avoid potential reenrichment costs. The DRACS coolant salt should, however, include neutron poisons to shut down the reactor in the case of significant heat exchanger tube leakage.

Since the DRACS is designed as a safety-related system in the FHR-TS, the fluidic diode has to be qualified for safety-grade nuclear service. Although fluidic diodes have been used in nuclear systems previously [38], a fluidic diode compatible with liquid salts at elevated service temperatures has not previously been demonstrated.

\subsection{INSTRUMENTATION AND CONTROL SYSTEM}

With the exception of neutron flux, high temperature process instrumentation applicable to the FHR has been developed and demonstrated in laboratory environments. However, little of the instrumentation is commercially available, and its longer-term reliability and drift performance have not been established. In general, the specialized high temperature tolerant, high reliability transducers and the supporting electronics are only available as designs from the literature. A sufficient market has not existed for commercial vendors to establish and maintain sources of supply for the specialized instrumentation.

In the short term, custom implementation of the primary process transducers is recommended. The new implementations can take advantage of prior transducer technology demonstrations while incorporating modern electronics into the instruments. The design rationale and operational performance information can then be captured to provide confidence in the use of the advanced transducers as reliable technologies. Because the reliability of these FHR-TS transducers will be uncertain, sufficient redundancy should be provided, as well as the capability to replace failed transducers. 
Fiber-optic couple pyrometry and Fizeau cavity-type thermometers are available commercially. The remaining issues with the technology are in the longer-term performance of the transducers under plant conditions and the stability and reliability of both the opto-electronics and their control logic. Ultrasonic wireline thermometry has been repeatedly demonstrated in harsh, nuclear environments. However, a high reliability, commercial implementation does not currently exist.

The required temperature for flux measurements at FHRs is roughly the same as those for high temperature gas reactors. Hence FHRs can take advantage of the developments being performed in the NGNP program. When the reactor is at power, ex-vessel (lower temperature) detectors are adequate for flux measurements. However, during start-up higher temperature tolerant, high sensitivity flux measurements are required. Commercial fission chambers are available to roughly $550^{\circ} \mathrm{C}$. Above this temperature the off-gas of the chamber metallic elements tends to re-deposit on the electrical insulators shorting out the detector. An improved detector design, incorporating higher temperature tolerant materials, would be necessary for start-up flux monitoring.

The instrumentation required to characterize the detailed chemical state of fluoride salt exists as laboratory-type instrumentation and is not readily available in an industrial format. Optical absorption spectroscopy is particularly useful in fluoride salts for identifying trace chemical constituents and their valence state. Electrochemistry is an alternate, well-known methodology for determining speciation within the fluoride salt. Optical access to the salt is most readily provided through windows in the pressure boundary. Diamond is well compatible with fluoride salts, and at lower temperatures sapphire is also a candidate window material. The high reactor temperature and the difference in coefficient of thermal expansion between prospective window materials and metals make immersed windows challenging. As an alternative, measurement access to the salt can be provided through a standpipe above the salt containing an inert gas bubble; the optical return path would be provided by a noble metal mirror within the salt. The standpipe would extend into a cooler region at which temperature window technology is readily available.

Gamma thermometers are based on the same technology as thermocouples, and so should have the same survivability characteristics. However, specific high-temperature versions of gamma thermometers have not been developed.

Clamp-on, ultrasonic flowmeters are a commercial technology in widespread use. High temperature standoffs to implement the flowmeter on very hot piping have been demonstrated for more than 30 years and can be ordered as a custom engineered component. However, as a custom component the long-term reliability under engineering service conditions has not yet been established.

Venturi flowmeters require accurate differential pressure measurement. Pressure measurement is often implemented as a diaphragm deflection measurement. Precious metal (or precious metal coated) diaphragms are compatible with fluoride salts. The pressure-sensitive diaphragm can be implemented at the distal end of a small diameter hollow tube. Optical fibers are commercially available with temperature ratings higher than FHR primary coolant temperatures. Interferometric methods are commercially available to measure diaphragm deflection at the distal end of an optical fiber. The central issue for the optical fiber coupled technique is to establish the long-term system reliability under actual service conditions. As an alternative to directly measuring the high temperature diaphragm deflection, an incompressible fluid (such as $\mathrm{NaK}$ ) can be employed to transfer the pressure from the high temperature bellows, along an impulse line, to a lower temperature diaphragm whose deflection can be measured using well-proven technology. 
The core inlet pressure of the annular-core FHR is sufficiently low that another method for measuring pressures inside the reactor vessel is with manometer lines, where the measurement of the liquid elevation would provide indication of the local pressure.

Mechanical float, bubbler, and radar-based level measurement technologies are all established commercial technologies. While custom implementations for a specific FHR variant would be useful, liquid salt level measurement technology is mature.

The pebble handling system will require instruments to measure pebble mass, mechanical soundness, and activity. These measurements will be similar to what is required at gas-cooled pebble bed reactors. Consequently, much of the required technology is near commercial maturity. However, FHR pebbles differ from those for gas-cooled reactors, and part of the recommended FHR instrumentation assessment needs to be devoted to FHR-specific pebble measurement implementation issues. 


\section{IDENTIFICATION OF COMPONENT TEST REQUIREMENTS}

The FHR reactor concept remains at an uneven level of development. Most systems have a viable candidate implementation. For example, the primary coolant pumps for an FHR will closely resemble those successfully implemented during the MSRE program. However, some important reactor aspects remain to be demonstrated. The power density of an FHR core can be 5-8 times as large that of a modular high temperature gas-cooled reactor. Coated particle fuel in the structured pebbles of a pebble bed FHR has not been demonstrated to perform adequately at this flux level. Moreover, the near-core graphite would accumulate radiation damage rapidly at FHR power levels. Radially profiling the core such that nonfueled pebbles are adjacent to the structural reflector graphite is important to the viability of the pebble bed FHR concept in order to avoid frequent graphite replacement. While structured motion of pebbles has now been shown with surrogate materials, demonstration of core pebble motion with prototypic materials under representative conditions is important to establish the viability of the concept.

None of the FHR systems or components has a final design implementation. Significant performance changes to the FHR-TS characteristics are likely as modern materials are applied to the FHR. Composite ceramics were much less well developed during the earlier MSR development effort. Higher temperature tolerant structural alloys are becoming available for reactor vessels and primary piping due to the HTGR program. The higher temperature alloys may increase the FHR achievable core outlet temperature by as much as $150^{\circ} \mathrm{C}$ over the earlier Hastelloy-N. Similarly, salt compatible thermal insulation was unavailable to the earlier development effort. The combination of the material developments makes possible more advanced reactor coolant structures than have heretofore been possible.

The nature of the component testing and the test requirements reflects the component and system maturity evaluation presented in Chapter 3. Specific testing needs are identified in this chapter for each evaluated system. The component testing needs are summarized in a prioritized list in Table 7. Only relatively near-term testing needs are included in the table. System level and long-term endurance testing will be required for all of the components. However, the FHR-TS design is as yet too immature to be able to prioritize the longer-term and system level tests. Also, while some materials testing will be performed using component test facilities, materials test planning is part of the FHR materials development planning process. The near-term tests are prioritized on a four-level scale: (1) vital-the performance of the component directly impacts the FHR concept viability, (2) high-the performance of the component significantly impacts FHR desirability, (3) medium - the test demonstrates important component technology impacting FHR desirability, and (4) low-the test demonstrates useful component technology.

\subsection{SUMMARY}

A complete reactor design does not yet exist for the FHR-TS. Until a complete reactor design is produced that integrates all of the required system functions, establishing a scored maturity evaluation remains inappropriate in that scoring could reasonably be expected to give a misleading impression of design maturity. For each system required testing is classified into research support (development or refinement), reestablishing competency, performance (refinement or proof testing), or reliability (proof testing).

Even with reactor design remaining to a significant degree undefined, reasonable estimates can be made of the constituent system performance requirements. Testing needs are driven by estimated system performance requirements. As the FHR-TS design matures, additional component testing needs will likely be uncovered. Thus this component testing report will periodically need to be revised and updated. Four types of testing are envisioned: 
1. Small-scale tests to evaluate performance at the subcomponent level which have been previously untested in the expected environment. This testing might include evaluation of new materials, evaluation of new designs or design features, testing under operating conditions or modes that were not examined previously, etc.

2. Functional testing at the component level to assure individual component performance.

3. Functional testing at the system level where component interactions will influence performance.

4. Long duration endurance testing to establish reliability and failure rates, both for investment protection, and to develop the required data to make the safety arguments necessary to license the reactor.

As the design of the FHR-TS develops, and understanding of the transient response increases, it may become desirable to perform some system testing using the facilities developed for component testing. The specific transient testing requirements will need to be developed as the design matures.

\subsection{REACTOR CORE SYSTEMS}

\subsubsection{Fuel Elements}

Only limited fuel element testing is required as part of a component test program. The fuel element component testing primarily needs to demonstrate that the pebbles move as planned through the core and that wear and radiation damage do not substantially alter their characteristics. Studies for pebble wear can be performed in separate facilities from those used to perform irradiation testing of fuel pebbles. Thermal/fluid modeling of the core region will also be required - both to characterize motion of the pebbles in the core, as well as to evaluate pebble heat transfer and ensure that fuel temperatures remain acceptable. Graphite dust in the salt due to pebble wear should also be investigated.

The purpose of the pebble mechanical testing will be to establish the pebble surface conditions and primary coolant salt lubricating properties across the range of operational temperatures and with full life accumulated radiation damage. Additionally, repeated pebble insertion and extraction from liquid salt need to be performed to demonstrate whether pebble surface graphite is vulnerable to pitting type damage.

Evaluating the structured pebble motion within the core will be performed initially in scaled experiments using water with high density polyethylene spheres, and subsequently will be a principal fuel element component test. The testing needs to demonstrate that pebbles inserted at specific radial locations within the core progress smoothly up through the core without clumping or adhesion and that no locations exist within the core where the pebbles may hang up or fail to move as anticipated. Local fluid motion and corresponding heat transfer characteristics can be characterized using surrogate fluids, with confirmatory testing in salt performed where critical.

Most pebble testing can be performed with flinak salt as the mechanical and heat transfer properties of fluoride salts are similar and follow known scaling rules. However, as the pebble motion is a key issue to the reactor core design, confirmatory testing of pebble lubrication properties and pebble cleaning/surface erosion needs to be performed using flibe. 


\subsubsection{Reactivity Control and Shutdown System}

\section{Design information needs}

A comprehensive concept for reactivity control and shutdown features, including fuel inventory control requirements, mechanically driven control and shutdown rods, reserve shutdown method, inherently operating shutdown features, and the use of other pebbles (fertile, blank graphite, or special absorber pebbles) is needed before specific test requirements can be identified. The discussion below identifies the general requirements and test features likely to be needed to support finalization of design, and later, to demonstrate the effectiveness and reliability of the reactivity control system. Experiments are expected to be performed at the Czech Nuclear Research Institute (NRI) zero-power test facility using MSRE coolant salt to validate models for negative coolant void reactivity.

\section{Modeling requirements}

Modeling of reactor kinetics will be needed to complete the design and evaluation of the control system. Modeling activities associated with pebble identification and assessment integrate with the fuel inventory control logic. Modeling of the overall performance of the instrumentation system used to assess reactivity and trigger operation of control and shutdown systems will be an integral part of the design of the control and shutdown hardware. Modeling of anticipated transients, such as xenon oscillations or turbine trips, should also be investigated to assure that the control system can handle these potential occurrences properly. These issues, however, are not considered within the scope of the component testing and are expected to be addressed during the conceptual design phase.

\section{Testing requirements}

Criticality testing, in a facility that models all applicable aspects of the reactor system, may be needed to benchmark and support the models used in the design of the control system. Possible features of the criticality test would involve the use of isotopically accurate primary coolant salt (if flibe, the fraction of ${ }^{7} \mathrm{Li}$ would be critical). Features for varying the temperature of the critical experiment about the normal reactor operating temperature may be needed to evaluate temperature coefficients; transfer of pebbles might be required to model control system responses, and modeling of other coefficients such as possible voids or other physical changes may also be important. Criticality testing is not within the scope of the component test facilities addressed in this report and will not be discussed further.

Tests will be needed to validate performance and durability of absorber insertion systems. Injection, defueling, and control of fuel inventory and the distribution of pebbles in the core will likely remain in the scope of fuel handling system test facilities. Testing of special pebble facilities used for control purposes, operated separately from the main fuel handling system, may be required as part of the reactivity control and shutdown program.

The first tests of developmental and prototype components of the reactivity control and shutdown system will likely meet designers' needs for information. Testing will likely be performed first reducedscale experiments with water that match Reynolds and Froude numbers, then at prototypic-scale at operating temperature and other key environmental conditions representative of reactor operation. Heat generation in the absorbers must be accounted for in planning these tests. For absorbers immersed directly in the salt, tests in a flowing salt environment may be required. Ultimately, durability testing will be required for the components of the reactivity control and shutdown system. Testing will include long duration evaluation of issues such as fatigue, wear, condensation of $\mathrm{BeF}_{2}$ in cold regions of the drive assembly, etc. using test stands dedicated to proving reliability of safety-related features. 
Other test requirements for the reactivity control and shutdown hardware will address operational applications, such as installation, on-line monitoring and in-service inspection, and remote maintenance. These tests requirements may be met as part of a larger program to demonstrate overall reactor operation and maintenance features and procedures.

Material development requirements may be required, based on the technological maturity of materials selected for absorbers, shafts or cables, guides, latches, positioning devices, monitoring, and other components. These tests may progress from tests on fiber and matrix materials, to small sample tests, to representative geometry tests, and finally to complete representative components tests. In some cases, irradiation testing may be required to demonstrate performance under representative irradiation conditions.

\subsubsection{Fuel Handling and Storage System}

\section{Design information needs}

The current status of the pebble handling system focuses on demonstrations of the ability to zone pebbles in the core using scaled experiments, and on select issues associated with pebble movement such as demonstrations of the lubricating effect of molten salt. A comprehensive design of the pebble handling system, even at the conceptual level, has not yet been documented.

\section{Modeling requirements}

Modeling of irradiation and decay will be needed to predict the fissile inventory, radioactivity, and decay heat in pebbles. Additional heat transfer models will be needed to provide a design basis for cooling of pebbles in the FHSS, and to identify the parameters, including cooling time in the defueling chute, for safe transfer from a molten salt environment to dry handling systems.

Extensive core physics modeling will be required to define the desired pebble loadings and zoning patterns for fuel, graphite, and possible fertile material pebbles. These models will also be used to determine the precision to which pebbles must be placed and the tolerance of the reactor to misplaced pebbles.

Modeling of the source term, radiation transport, and detector performance will be needed to develop the characterization system used to assay pebble burnup, including both fuel and fertile material content.

Hydraulic modeling will be needed to predict (1) pebble behavior as pebbles are being fed into the reactor core by the salt system, (2) possible impacts on proper positioning of pebbles as they are initially inserted into the core and as the pebble bed passes through the core, and (3) cooling and movement of the pebbles once inside the core. Likewise 3-D computational fluid dynamics will be needed to predict the coolant temperature distribution in the reactor outlet plenum under forced and natural circulation flow conditions, and to predict the peak temperatures of structures in contact with the exiting salt and the mixing processes that equilibrate the salt temperature to the bulk temperature before it reaches the primary pumps.

Additional modeling will be used to determine specific reactivity impacts in special regions as required, such as assessing the possibility of incorporating the placement of fertile material pebbles in regions of high reactivity as a feature of the reactivity control system. 


\section{Testing requirements}

General testing of the fuel pebble handling system will be needed as the overall design concept evolves. Initially, test requirements will be met by simple scaled systems such as those now in place at UCB. Eventually, it is possible that a dimensionally accurate, full-scale overall system may be required to demonstrate all transfers and evaluate pebble distribution in the core and possible locations for pebble trapping or holdup and locations of reduced flow and heat transfer. These tests may use water or surrogate pebble/fluid combinations to simplify construction of the facility.

As the design of the pebble handling system evolves, testing in a salt environment will likely be required. Certain key areas, such as introduction of the pebbles into the core, may require testing in a fullscale, salt-filled mockup at the planned operating temperature and flow rate. Such testing may be integrated with pump and other component tests. Additional testing will be performed as part of start-up testing of the FHR-TS reactor; however, this start-up testing in the FHR-TS must be largely confirmatory since the capacity to modify the FHR-TS design will be very limited at that point.

Test requirements for pebble handling in the gas-filled portion of the system will be simpler. Much information can be gained by observing tests conducted on HTGR systems; specific tests of the FHR-TS systems may be accomplished by adapting existing facilities, or with new facilities based on experience gained with gas-cooled reactor pebble handling test facilities.

A key element of the FHSS is the identification of individual pebbles as part of the return/remove decision process and to track fuel inventory in general. Specific test requirements will be developed as the operational requirements and design concepts evolve. A key question at present is whether identification will be performed in a salt or gas environment. Testing will initially be performed under ideal conditions, then move to assess identification capabilities under degraded conditions (covered with salt, damaged identification markers, etc.).

Testing of the system for assessment of burnup in individual fuel and fertile material pebbles will similarly progress from bench-scale tests of simulated fuel and fertile material to the possibility of field tests with pebbles irradiated in test reactors. Again, specific test requirements will evolve as the requirements for the system are defined, with a significant amount of information for the design and operation of the detection and selection systems derived from pebble bed gas-cooled reactor system designs.

Physical characterization of pebbles is needed to prevent damaged fuel pebbles from being returned to the core, even if their cycling history and burnup indicate that they should be returned. Test requirements may parallel those of the identification systems; testing could move from initial tests under ideal conditions to tests in more challenging environments.

Test requirements may address the capability of the system to identify and locate any pebbles that may not be circulating properly. In particular, a system may be needed to determine if and where pebbles may be trapped in the core or in portions of the FHSS. Test requirements will evolve as the concept for inventory management and overall assessment of FHSS operation is developed, and will likely link with the pebble identification and tracking system and with tests of instrumented mockups of the overall FHSS.

Long duration testing of the pebble handling system at the subscale level will be needed to assure system reliability, pebble and reflector wear due to the handling process, and development of maintenance requirements and procedures. 


\subsection{HEAT TRANSPORT SYSTEM}

\subsubsection{Reactor Vessel and Primary Piping}

The reactor vessel and primary piping are primarily material structural elements and thus generally outside the scope of component testing. However, they do function as integral parts of the primary heat transport system, and the salt flow characteristics may be determinative of their long-term survival. Also, since the layers of the reactor vessel and primary piping function as a system, integrated system performance testing will be necessary to provide confidence in their combined performance.

The largest operational uncertainty in the layered vessel structure is in the performance of the insulation layer, as protecting the exterior pressure vessel from overheating is a safety function. The required system performance of salt-compatible insulation has not yet been established. Relatively small cracks in the insulation could allow the buffer salt to establish a thermal convection loop and transfer heat effectively to the exterior vessel. However, the salt viscosity increases greatly as it cools, converting to a viscous, glassy material that is itself a thermal insulator. Thus to a large extent cracks within the insulation layer may be self-healing, and it may be advisable to employ a frozen salt layer directly on the guard vessel to limit ability of the liquid buffer salt to transfer heat to the reactor vessel.

Significant modeling, material design, and testing remain before the FHR-TS vessel material system can be selected. Initially, laboratory-scale testing with prototypic materials is recommended to support the material design and system assembly modeling. Later, larger-scale performance verification testing will be required under representative hydraulic and thermal conditions. As part of the performance verification testing, demonstration of buffer salt flow into the primary vessel under interior vessel faulted conditions will also need to be performed.

The siphon break element of the primary piping will also be a safety-grade component in the primary heat transfer loop as it prevents the primary coolant salt level from being lowered beyond design limits for faulted salt inventory. While loss of prime to the primary pump can be accomplished via several passive, highly reliable implementations, the siphon break will need to be demonstrated as part of system viability testing and during performance testing.

Beyond the siphon break, the FHR-TS primary piping may not be considered important to safety since once the siphon break has activated, the reactor pool is hydraulically isolated from the primary piping. However, the primary piping is used for normal shutdown and is vital to reactor performance. Primary piping performance will be inherently demonstrated as part of component testing since salt flow into and out of test articles will of necessity be with similar piping. As the primary salt is at low pressure and some candidate primary pipe materials are difficult to weld, pipe segments may be joined with mechanical flanges. Flange and accompanying gasket performance testing would also be necessary at both laboratory scale in support of design and at larger scale in support of performance verification.

\subsubsection{Liquid-Salt Pumps}

The development of the FHR-TS pumps will entail the testing of pumps and certain individual pump components. The results of these tests will be used to refine the design specifications. Some commercial capability exists to construct smaller salt pumps.1

1 Teikoku Electric Mfg. Co., Ltd., Japan, manufactures pumps with the following capabilities: $6-\mathrm{m}^{3} / \mathrm{h}$ max flow, 30 - $\mathrm{m}$ head, $75 \mathrm{~kW}(100 \mathrm{hp})$ power, $400-800^{\circ} \mathrm{C}$ temperature operating range. 
Different pump configurations may be evaluated as the design matures. The current baseline design uses short-shaft pumps located on the reactor and intermediate loop hot legs. However, a long-shaft pump configuration may also be an attractive candidate for pump selection based on the comprehensive design evaluations conducted prior to the cancellation of the MSBE and MSBR programs.

Liquid fluoride salt pumps would also be useful in other energy generation technologies, such as solar power tower applications, which currently use the nitrate salts as both a heat transport medium and heat storage medium [26]. The FHR-TS pump development program can therefore also leverage on the technical know-how and operating experience outside the nuclear field.

Primary pump test requirements are identified in three major categories: design related, operation related, and safety related. Design-related tests are those that deal with certain parts of the component. For the primary coolant pump, the major design-related tests are (1) hydraulic design, (2) rotor dynamic analysis, (3) material, (4) bearings and sleeves, and (5) seals. Once the selected pump design reaches a certain maturity level, the component will be subjected to performance and endurance testing. The performance testing is intended to ensure that the nominal operational objectives such as specific speed, pressure, and head are achieved. Endurance testing is intended to ensure that the component reliably operates at the nominal operating conditions (e.g., pressure, temperature, speed) for an extended period of time. The endurance testing may also involve load cycling, including start and stop operations, temperature variations, pump speed control, etc.

\section{Hydraulic Design}

A base hydraulic design will be selected that will provide the required head and coolant capacity at a reasonable shaft speed and net positive suction head.

\section{Rotor Dynamic Analysis}

Understanding of principal vibration modes of the long shaft at various pump speeds is crucial to extending the life of the bearings and operating the pump reliably. The location and performance characteristics of shaft dampers and bearings necessary to provide a safe margin of fatigue life for the shaft should be determined as a function of shaft diameter (as per p. 12 in [5]).

\section{Material}

It is critical that the thermal expansion of the rotating assembly and stationary components of the pump match to prevent distortion and binding [39].

Temperature cycles can impose increments of plastic strain in the high-temperature portions of the pump due to changes in either thermal stresses associated with steep temperature gradients or mechanical stresses associated with pipe anchor forces and moments exerted on pump nozzles (p. 13 in [5]). The total plastic strain in various parts of the pump must be estimated for a specified number of cycles. Measures must be taken to keep the total strain within the plastic fatigue limit of the container material.

\section{Bearings and Sleeves}

The operating stability of the bearing and the start-stop wear resistance of bearing materials will be verified in a component test as was done for the MSRE (p. 14 in [5]). Different bearing and sleeve materials can be tested in a single test assembly that accommodates multiple bearings. The appropriate bearing configuration can first be operated at room temperature with a fluid having the approximate kinematic viscosity of the salt to ensure stable operation of the bearing. This first test will be followed by 
operation of the bearing in liquid salt for more than the anticipated start and stop cycles with similar operating temperatures. The bearing will then be subjected to thermal cycling over the anticipated temperature range of the FHR-TS. Performance characteristics of the pump will be continuously observed at each juncture. Finally, the bearings will be operated for extended periods to observe the endurance characteristics.

Similar performance and endurance testing for proper bearing design and materials have been underway at other research laboratories and industry $[26,40]$.

\section{Seals}

As the specific pump seal designs are developed, they will require performance, reliability, endurance, and safety focused testing. Much of seal testing will focus on rotating dry gas seals, which have been developed since the cancellation of the prior MSR pump development efforts, under FHR operating and accident conditions. If a liquid lubricant is isolated from the primary fluoride salt coolant with a seal, safety evaluation will be particularly key in that organic materials have the potential to leak into the primary coolant, thermally decompose, and potentially obstruct reactor cooling.

\section{Integrated Pump Tests}

The mature pump design will undergo several tests at room temperature using a suitable fluid, including (1) checking the hydraulic design; (2) developing appropriate controls for required bypass flows through the pump tank; (3) providing adequate capacity for degassing the liquid; and (4) determining the adequacy of the pump design to meet special, transient, or emergency conditions that can be encountered in reactor operation. Long duration pump testing will be used to develop reliability data and required licensing information. These tests will be conducted using salts at operating temperature and will utilize test stands that can be shared with other components. Accelerated durability testing can be incorporated into the test program if schedules require it.

\subsubsection{Intermediate Heat Exchangers}

The use of conventional heat exchanger designs such as were used during the MSRE program, and which are very common industrial designs, would minimize the test program necessary to verify design performance. Although several issues arose with the MSRE heat exchanger at the time, significant advances in design tools have been made since 1961 (the time of the MSRE design), and it is expected that these would significantly decrease the risk of designing a heat exchanger that does not perform as expected. The MSR program has not been actively developing large components for over 35 years, and some of the technology developed at that time would have to be recovered. This would include technologies such as the tube to tube-sheet joint used in the MSRE heat exchanger, and forming tubes and thick cross sections out of the selected material. Likewise, new connection methods will need to be developed if clad tubing is used.

Small-scale development and testing will be required to verify such things as tube to tube-sheet joint integrity and salt heat transfer performance. Larger-scale testing will be needed in a component test facility for heat exchanger performance evaluation. A testing program would include both thermal performance evaluation that might be relatively short in duration, as well as long-term durability testing under prototypic molten salt conditions. Initial testing could begin with surrogate fluids and progress to inserting the heat exchanger in a molten salt loop. A scaled heat exchanger could be used for both thermal performance and long-term durability testing if design features were included, allowing the incorporation of appropriate instrumentation. 
To support heat exchanger testing, the component test facility will be required to provide molten salt at reactor operating temperatures and pressures. Flow rates must be sufficient to maintain molten salt velocities within the heat exchanger at reactor values, and available pressure head must be at least the design value of the primary system heat exchanger. Secondary side salt flow, pressures, and temperatures must similarly be those of the reactor system. Instrumentation included in the component test facility must include sufficient temperature, pressure, and flow measurements to be able to perform primary and secondary energy balances, and measure or infer flow-induced vibration within the heat exchanger. Local measurements (within the heat exchanger) may be required to determine local heat transfer, internal flow distribution, and local accelerations.

If unconventional heat exchanger designs were chosen (either materials or geometry), additional emphasis would have to be placed on small-scale testing and development that concentrated on unique fabrication techniques that might be necessary and on thermal hydraulic performance testing (heat transfer, pressure drop, vibration, etc.). This development would need to include both surrogate fluid experiments as well as molten salt testing at prototypic conditions. Testing in a component test facility would be utilized for testing similar to that described above for conventional heat exchanger designs.

\subsubsection{Secondary Heat Exchanger}

Salt-to-gas heat exchangers are important to the FHR reactor class. A design and development effort will be needed before a design is finalized. Multiple options have been identified as design possibilities, including plate and printed circuit-type heat exchangers. The design of this heat exchanger has significant consequences on the power conversion cycle in that both temperature drop across the heat exchanger and pressure drop across the gas side of the heat exchanger impact efficiency. Conventional design analysis can be used for the thermal and fluid design of these systems, including thermal stress analysis for steadystate and transient response. Structural and vibration analysis will also be needed to assure the reliability.

Testing will be needed to ensure the thermal, fluid, and structural performance for these designs. This testing can be initially conducted using surrogate fluids, with follow-on testing in salts. Testing using scaled designs can be used to characterize heat transfer performance at scaled heat loads and at prototypic temperatures. Long duration hydraulic testing of the designs can be conducted in concert with pump testing. Instrumentation requirements for this testing will be similar to those discussed for the IHX, along with a gas supply sufficient to duplicate those in the power conversion system.

\subsubsection{Salt Processing and Inventory Control System}

The PICS for the FHR-TS has not yet been designed. Consequently, testing requirements for the PICS remain at a conceptual level, and specific methodologies and facilities cannot yet be provided. The individual processes for salt chemistry control were developed and demonstrated during the MSR program. Testing, however, is required as part of the technology demonstration phase of FHR development. Salt cleanliness control and chemical processing require more complex handling of the salts than any other reactor system. As such, system demonstration is required to re-familiarize system designers with the details of salt handling and transferring.

The primary salt will be somewhat radioactive, tritium contaminated, and toxic due to its beryllium content. Process automation for the salt chemistry control is therefore important. As process automation technology has greatly improved since the prior MSR development campaign, salt measurement and cleaning instrumentation and controls technology will need to be demonstrated. Additionally, different salt contact materials are likely to be used in an FHR than in the MSRE, resulting in different salt contaminants. Sensor technology has also progressed over the intervening years. Consequently, the salt 
penetrations allowing the new transducers to access to the coolant salts will need to be verified. Diamond-coated optics and electrical penetrations were not practically available during the earlier development efforts, and on-line salt condition monitoring would provide significant confidence that significant corrosion has not progressed significantly.

In general, most testing can be performed at laboratory scale with nonberyllium-bearing salt as the heat transfer and mechanical properties of fluoride salts are similar and follow known scaling rules. However, chemical treatment of salt will need to be performed using flibe and eventually at representative scale.

\subsubsection{Cover Gas Chemistry and Pressure Control System}

Most aspects of the cover gas and pressure control system use mature commercial technology and as such do not require testing. The two notable exceptions to the high level of design maturity are ultrasonic degassing of fluoride salts and the subsequent chemical trapping of the evolved tritium. Neither ultrasonic tritium removal system nor the tritium trapping system has yet been designed for the FHR-TS. Tritium trapping technology is relatively well known, thus performance demonstration will therefore await FHR-TS cover gas system design to enable the testing to be representative of the plant requirements. Ultrasonic degassing testing will initially be in support of technology development, as ultrasonic degassing technology has not been previously applied to liquid fluoride salts. Because the intermediate and secondary heat exchangers have large surface areas and permeability to tritium, and the primary and intermediate salts have much lower solubility for tritium than the power conversion system gas, tritium control may also require recovery from the power conversion system.

\subsubsection{Valves for Liquid Salts}

\section{Mechanical valves}

The development of fast-acting isolation valves would be essential to the traditional application of isolation valves for the secondary coolant piping passing through the containment boundary. Current freeze valve technology is not applicable to service as flow control or fast-acting isolation valves. If the containment concept used for the FHR does require fast isolation valves, development of mechanical valves is likely to be necessary. Because these isolation valves would only have to operate under highly infrequent accident conditions, they could be designed for one-time operation (for example, using an explosively-activated clamp to crush the pipe closed).

More convential shutoff valve approaches could include fully sealed shafts (for example, a sealed bellows capable of being filled with liquid salt or another buffer fluid) or a nonmechanical coupling between the actuator and the valve internals (such as a magnetic coupling).

The initial test facility could operate in a static system, with molten salt at system design pressure. Such a test would allow development and durability testing of valve seats and actuator systems. As valve technology is developed, testing of valve operation at flow conditions would be required.

\section{Freeze valves}

The use of freeze valves to establish shutoff in small diameter lines in a static salt system is an established technology. Careful analysis is required to ensure that stresses and fatigue resulting from changes in temperature and salt density do not lead to freeze valve failure. Testing of specific freeze valve designs may be appropriate to confirm operating characteristics and to measure stress and strain upon 
repeated cycling. These tests could be performed on dedicated test stands with capability of adding and removing salt from the valve itself, or they could be integrated into a larger loop facility.

If concepts are proposed for fast actuation of freeze valves in a static environment, or for operation of freeze valves with flowing salt, further testing would be required. Specific test requirements, including the appropriate scale of test facilities, would be developed as concepts are identified.

\subsection{DIRECT REACTOR AUXILIARY COOLING SYSTEM}

The DRACS in FHRs will be a primary safety system. One purpose for the FHR-TS is to demonstrate that the DRACS will perform its designated safety functions under a wide range of accident conditions. Integrated DRACS system testing is beyond the scope of this report and will need to be addressed following completion of the FHR-TS conceptual design. Initial testing of DRACS system performance is being performed using Dowtherm ${ }^{\mathrm{TM}}$ as a simulant fluid in the FHR Compact Integral Effects Test (CIET) facility at UCB. DRACS component testing is therefore oriented towards providing the design information required to complete the FHR-TS thermal and hydraulic design.

The FHR-TS DRACS will provide as much as 5\% total heat removal capacity of rated reactor thermal power. For larger plants the heat rejection would be distributed among multiple redundant DRACS loops. Constructed entirely of passive components-i.e., heat exchangers, a fluidic diode and pipes, and having a safety function for FHR class reactors - the DRACS system warrants a more holistic analysis even during early stage design. Since no moving parts are involved, testing requirements on DRACS mostly revolve around phenomenological demonstration, primarily the demonstration of establishing and maintaining the natural circulation mode in the primary loop and within the DRACS heat exchangers under the faulted conditions produced by all anticipated events.

At the component level, the primary testing focus is the performance of the fluidic diode and for heat transfer coefficients for the DHX and NHX heat exchangers. The fluidic diode is operational in the reverse flow mode during normal reactor operation primarily to keep the DRACS salt at liquid state as well as to maintain the circulation in the DRACS loop. The latter is important in assuring that, in the event of a loss of forced flow, the DRACS will be immediately available to provide sufficient heat removal from the primary salt. The thermal dynamics of the primary coolant will be dependent on how rapidly the natural flow will be established in the pool around the DHX as the pool bulk temperature increases.

Though fluidic diodes have been widely used in safety applications, the operation of a fluidic diode at representative conditions of the FHRs has not been demonstrated. Information and data obtained from this experience will be important in providing assurance that fluidic diodes can be designed and operated reliably with liquid fluoride salts over a wide range of temperatures.

\section{Design information needs}

Several different fluidic diode designs are possible as described in Sect. 2.4. The fluidic diode type and design need to be finalized prior to beginning component testing. Also, the DRACS coolant salt must be selected to provide definitive guidance on system testing. Candidate heat transfer salts were assessed by D. F. Williams in 2006 [16]. The most important parameter for the candidate salt is its melting point. A lower melting point reduces the potential for salt freeze-up and simplifies the design of support systems such as trace heating to prevent or mitigate such a condition. Further trade studies may be necessary to finalize the salt selection.

Other essential information necessary for a complete DRACS design is the base material for the construction of the heat exchangers - that is, the DHX and the NHX, the fluidic diode, and the connecting 
pipes. The selection of the base material is dictated by salt compatibility in both the primary side and inside the DRACS loop. Although the selection of materials is part of a larger materials program, experience gained from the component testing facilities will most likely provide significant insight for finalizing the material selection process.

\section{Modeling requirements}

Heat exchanger design for almost any operating environment is a mature field. Design of the DHX and the NHX will follow the design and construction rules of heat exchangers and provisions of other applicable requirement in designing a safety-related system. The operating experience and the maturity of available designs will be taken into account during the selection of heat exchanger type. As the shutdown cooling system, EBR-II employed a bayonet heat exchanger that functionally acted as the DHX. The performance of the system was demonstrated successfully for a number of accident scenarios in real experiments. There have been preliminary design studies for the DHX and NHX that used shell-and-tube type heat exchanger [4].

The DHX will be designed to have significantly lower thermal resistance than the NHX, so that the temperature drop from the primary salt to the DRACS salt will be significantly smaller than the drop from the DRACS salt to air, to prevent the potential for salt freezing. Since the DHX is fully immersed in the primary liquid salt that is near to the temperature of the DRACS salt, it has relatively more uniform temperature distribution (i.e., heat transfer from a high-temperature fluid to high-temperature fluid); hence it is subjected to relatively less thermal stress. However, the NHX will experience a drastic temperature gradient due to its designated function of transferring heat from a high-temperature fluid to air essentially at the ambient temperature over a wide range of environmental conditions. Therefore, accurate modeling and representative testing of the component at realistic conditions must be conducted to confirm safe operation. Confirmatory testing can be performed with a small-scale component; however, stress analysis must be conducted in a representative scale.

Computational fluid dynamics simulations of vortex diodes, a type of fluidic diode, have been recently reported in the literature [23]. Further computational studies might be necessary to capture optimal design parameters in relation to other systems. Diodicity, which is defined as the ratio of forward flow to reverse flow pressure drop, should be maximized so that the bypass flow under forced circulation conditions is limited to a few to several percent of the total flow, comparable to the natural circulation flow that occurs under shutdown conditions for decay heat removal. The performance of the loop (i.e., sustaining the natural circulation) must be monitored on a regular basis. Any departure from designated operating envelope of the system must be used to initiate the support systems such as closing the air-flow dampers, and if this is not sufficient, activating trace heating to prevent DRACS coolant salt freeze-up.

The amount of trace heating is a function of the rate of heat loss from the NHX to the atmosphere, which is inversely related to the ambient temperature. Therefore, the amount of trace heating is determined by the lowest seasonal temperature of the location where the FHR-TS will be built. This seasonal low will also be important in performing stress prediction calculations of the NHX.

During the normal operation of the reactor, resistance through the reactor core will change based on the number of fuel elements, which might be adjusted for controlling the power level. The reactor power is also planned to be regulated by adjusting the primary liquid salt flow rate, thus changing the specific velocity of the coolant. Variations in specific velocity will result in different levels of change in frictional head loss through the core and the fluidic diode. These effects must be well understood by accurate computational models. These models might demonstrate unanticipated phenomena and provide significant insights for the component design. 


\section{Testing requirements}

The most important DRACS component tests are demonstrations that the natural circulation loop can remove heat at the design rate with the design temperature drop, and demonstration that the fluidic diode will perform its intended function for a variety of operating conditions.

The DRACS will operate continuously during the normal FHR-TS conditions in reverse direction, at reduced flow. Air flow control louvers will be in their closed setting, which will restrict the air flow to the quantity needed to maintain the concrete shield structure below its maximum design temperature. The amount of the reduced flow and thus the rejected heat is determined by the reverse flow resistance characteristics (or diodicity) of the fluidic diode. The normal operation condition heat transfer will be sized to maintain the DRACS cooling loop in a liquid state. In the event of a loss of flow in the primary heat transport system, such as primary pump failure, primary coolant flow in the DRACS ducting will reverse direction. The cold salt that fills the DRACS channel has significantly higher density that the hot salt in the core and core outlet plenum, and thus it immediately begins to flow downward when the forced-circulation pressure drop across the core disappears. The resultant increase in core bottom pressure in turn causes the primary coolant to rise through the core, establishing a natural circulation path in the pool around the DHX and through the fluidic diode in its low flow resistance direction. The fluidic diode's performance during the flow reversal transition must be computationally investigated and experimentally demonstrated to provide adequate confidence that the DRACS cooling loop will start up as required.

The fluidic diode must also be tested for long-term operation to demonstrate that the component will meet its functional requirements with minimal corrosion or fouling. As with other material corrosion vulnerability testing, special focus will be on the interaction of the chemistry control processes in the liquid salt and the performance of the fluidic diode. The fouling and corrosion issues for the DRACS components should be examined, and proper chemistry control practices must be addressed. These tests and the chemistry control measures are undoubtedly contingent upon the selected base material and the DRACS coolant.

The NHX design needs to be tested against the inclement weather conditions (e.g., snow, hail, very low temperature). In determining the design parameters of the stack, these conditions must be taken into account to minimize impact on NHX operation and structural integrity.

\subsection{INSTRUMENTATION AND CONTROL SYSTEM}

Apart from source and intermediate range flux measurements where technology development is still ongoing, FHR instrument testing is recommended to focus on establishing system stability and reliability. Reliable instrumentation will be required to support testing of the other heat transfer components of the FHR power generation loop. Hence it is recommended that process instrument development and testing be implemented in an early development phase to support the overall testing program (Table 7).

The FHR process instruments can most easily be tested in surrogate environments. Nonwetted instruments can be demonstrated without requiring a salt loop in standard laboratory equipment. The specific implementations of the ex-core temperature measurements will need to be subjected to a moderate gamma, high-temperature environment to establish reliability. As the core technologies of the temperature measurements have established performance histories under extreme radiation environments, the necessary system reliability testing would be focused on demonstrating that the FHR-specific implementation has not introduced any unanticipated vulnerabilities.

All of the FHR flow and level measurement instruments are applicable to lower temperature fluids where alternate instrumentation is readily available. It is therefore recommended to establish 
instrumentation calibration in a water loop before installing the instruments into a salt testing environment. Salt testing performance would be focused on establishing stability of response through longer-term operation rather than instrument calibration, as reference instrumentation is not readily available for high-temperature fluoride salt environments. Additionally, long-term testing will be needed to establish instrument reliability and failure data that will be required to develop licensing arguments.

Table 7. Summary of FHR-TS near-term component testing requirements

\begin{tabular}{|c|c|c|c|c|}
\hline SYSTEM & COMPONENT & TEST DESCRIPTION & SCALE AND FACILITIES & PRIORITY \\
\hline \multirow{8}{*}{ REACTOR CORE } & \multirow[t]{4}{*}{ Fuel Elements } & \multirow[t]{2}{*}{ Fuel element motion } & $\begin{array}{l}\text { Surrogate materials-small and } \\
\text { prototypic scale }\end{array}$ & \multirow[t]{2}{*}{ High } \\
\hline & & & Fluoride salt — small scale & \\
\hline & & Fuel element cleaning & Flibe-small scale & High \\
\hline & & Fuel element lubrication & Flibe-small scale & High \\
\hline & $\begin{array}{l}\text { Reactivity Control } \\
\text { and Shutdown } \\
\text { System }\end{array}$ & $\begin{array}{l}\text { Poison injection module } \\
\text { performance }\end{array}$ & Furnace and endurance testing & Low \\
\hline & \multirow{3}{*}{$\begin{array}{l}\text { Fuel Handling and } \\
\text { Storage System }\end{array}$} & Fuel insertion and removal & Surrogate materials- $40 \%$ scale & High \\
\hline & & & Fluoride salt—low throughput & \\
\hline & & $\begin{array}{l}\text { Pebble mechanical } \\
\text { inspection }\end{array}$ & Fluoride salt — no radiation & Medium \\
\hline \multirow{13}{*}{$\begin{array}{l}\text { HEAT TRANSPORT } \\
\text { SYSTEM }\end{array}$} & \multirow{3}{*}{$\begin{array}{l}\text { Reactor Vessel and } \\
\text { Primary Piping }\end{array}$} & Siphon break & Fluoride salt-small scale & Vital \\
\hline & & Layered system performance & Fluoride salt-small scale & High \\
\hline & & Flange and gasket mating & Fluoride salt—small scale & Low \\
\hline & Liquid Salt Pump & $\begin{array}{l}\text { Performance and } \\
\text { subcomponent testing }\end{array}$ & Fluoride salt- - small scale & Medium \\
\hline & \multirow[t]{2}{*}{$\begin{array}{l}\text { Intermediate and } \\
\text { Heat Exchanger }\end{array}$} & $\begin{array}{l}\text { Pressure drop and heat } \\
\text { transfer }\end{array}$ & $\begin{array}{l}\text { Surrogate materials-small scale } \\
\text { Fluoride Salt -small scale }\end{array}$ & Medium \\
\hline & & Scale and fouling & Flibe & Low \\
\hline & \multirow{3}{*}{$\begin{array}{l}\text { Secondary (salt-to- } \\
\text { gas) Heat } \\
\text { Exchanger }\end{array}$} & \multirow{3}{*}{$\begin{array}{l}\text { Thermal structural, pressure } \\
\text { drop, and heat transfer }\end{array}$} & Surrogate fluids - small scale & \multirow[t]{3}{*}{ Vital } \\
\hline & & & Fluoride salt—small scale & \\
\hline & & & Fluoride salt-prototypic scale & \\
\hline & $\begin{array}{l}\text { Salt Processing } \\
\text { and Inventory } \\
\text { Control System }\end{array}$ & $\begin{array}{l}\text { Transducer penetration - } \\
\text { windows and electrical } \\
\text { feedthroughs }\end{array}$ & Fluoride salt-small scale & Medium \\
\hline & \multirow{2}{*}{$\begin{array}{l}\text { Cover Gas } \\
\text { Chemistry and } \\
\text { Pressure Control } \\
\text { System }\end{array}$} & \multirow{2}{*}{$\begin{array}{l}\text { Tritium removal via } \\
\text { ultrasonic degassing and } \\
\text { sparging }\end{array}$} & $\begin{array}{l}\text { Fluoride salt-small scale non- } \\
\text { radioactive materials }\end{array}$ & \multirow[t]{2}{*}{ Medium } \\
\hline & & & Flibe_-small scale with tritium & \\
\hline & Salt valves & $\begin{array}{l}\text { Operation of fast acting } \\
\text { mechanical valves }\end{array}$ & Fluoride salt-small scale & Medium \\
\hline \multirow{2}{*}{$\begin{array}{l}\text { DIRECT REACTOR } \\
\text { AUXILIARY } \\
\text { COOLING SYSTEM } \\
\end{array}$} & \multirow[t]{2}{*}{ Fluidic Diode } & \multirow[t]{2}{*}{$\begin{array}{l}\text { Wide range performance } \\
\text { testing }\end{array}$} & $\begin{array}{l}\text { Surrogate materials-small and } \\
\text { prototypic scale }\end{array}$ & \multirow[t]{2}{*}{ Vital } \\
\hline & & & Fluoride salt-small scale & \\
\hline \multirow{2}{*}{$\begin{array}{l}\text { INSTRUMENTATION } \\
\text { AND CONTROL }\end{array}$} & \multirow[t]{2}{*}{$\begin{array}{l}\text { Transducer } \\
\text { Elements }\end{array}$} & $\begin{array}{l}\text { Electrochemical impurity } \\
\text { measurements }\end{array}$ & Flibe_-small scale & Medium \\
\hline & & $\begin{array}{l}\text { Optical impurity } \\
\text { measurements }\end{array}$ & Flibe_-small scale & Medium \\
\hline
\end{tabular}




\section{REFERENCES}

1. C. W. Forsberg, P. Pickard, and P. F. Peterson, "Molten-Salt-Cooled Advanced HighTemperature Reactor for Production of Hydrogen and Electricity," Nuclear Technology, 144, 289-302 (2003).

2. University of California-Berkeley, Department of Nuclear Engineering Modular PB-AHTR System Description, Revision A, April 2009.

3. D. Caron et al., A Modular Pebble-Bed Advanced High Temperature Reactor, Report UCBTH-08-001, NE-170 Senior Design Project, University of California-Berkeley Thermal Hydraulics Laboratory, May 2008.

4. D. T. Ingersoll, C. W. Forsberg, and P. E. MacDonald, Trade Studies for the Liquid-SaltCooled Very High-Temperature Reactor: Fiscal Year 2006 Progress Report, ORNL/TM2006/140, Oak Ridge National Laboratory, February 2007.

5. D. Scott and A. G. Grindell, Components and Systems Development for Molten-Salt Breeder Reactors, ORNL-TM-1855, Oak Ridge National Laboratory, June 1967.

6. R. C. Robertson, MSRE Design and Operations Report, Part I-Description of Reactor Design, ORNL-TM-728, Oak Ridge National Laboratory, January 1965.

7. P. G. Smith, Experience with High-Temperature Centrifugal Pumps in Nuclear Reactors and Their Application to Molten-Salt Thermal Breeder Reactors, ORNL-TM-1993, Oak Ridge National Laboratory, September 1967.

8. A. G. Grindell and C. K. McGlothlan, Conceptual System Design Description of the Salt Pump Test Stand for the Molten Salt Breeder Experiment, Oak Ridge National Laboratory, ORNLTM-2643, August 1969.

9. M. W. Rosenthal, P. N. Haubenreich, and R. B. Briggs, The Development Status of MoltenSalt Breeder Reactors, ORNL-4812, Oak Ridge National Laboratory, August 1972.

10. L. E. McNeese et al., Program Plan for Development of Molten-Salt Breeder Reactors, ORNL-5018, Oak Ridge National Laboratory, December 1974.

11. J. W. Collins, V. F. Tonc, and G. A. Gibbs, Next Generation Nuclear Plant Project Technology Development Roadmaps: The Technical Path Forward for 750-800 ${ }^{\circ} \mathrm{C}$ Reactor Outlet Temperature, INL/EXT-09-16598, Idaho National Laboratory, August 2009.

12. M. Kambe and M. Uotani, "Design and Development of Fast Breeder Reactor Passive Reactivity Control Systems: LEM and LIM," Nuclear Technology, 122, 179-195 (May 1998).

13. P. Bardet et al., "The Pebble Recirculation Experiment (PREX) for the AHTR," pp. 845-851 in Proceedings of Global 2007, Boise, Idaho, September 9-13, 2007.

14. W. R. Grimes, Chemical Research and Development for Molten-Salt Breeder Reactors, ORNL-TM-1853, Oak Ridge National Laboratory, June 1967, pp. 102-132. 
15. D. F. Williams, L. M. Toth, and K. T. Clarno, Assessment of Candidate Molten Salt Coolants for the Advanced High Temperature Reactor (AHTR), ORNL/TM-2006/12, Oak Ridge National Laboratory, March 2006.

16. D. F. Williams, Assessment of Candidate Molten Salt Coolants for the NGNP/NHI HeatTransfer Loop, ORNL/TM-2006/69, Oak Ridge National Laboratory, June 2006.

17. P. G. Smith, Development of Fuel-and Coolant-Salt Centrifugal Pumps for the Molten-Salt Reactor Experiment, Oak Ridge National Laboratory, ORNL-TM-2987, 1970.

18. H. J. Lim and P. F. Peterson, Conceptual Design of the Intermediate Heat Exchanger (IHX) for the PB-AHTR, Report UCBTH-09-005, Department of Nuclear Engineering, University of California-Berkeley, May 2009.

19. P. F. Peterson, Capillary Tube and Shell Heat Exchanger Design for Helium to Liquid Salt Heat Transfer, Report UCBTH-07-003, University of California-Berkeley, May 2007.

20. R. B. Briggs, "Tritium in Molten-Salt Reactors," Reactor Technology, 14(4), 335-352 (Winter 1971-1972).

21. D. T. Ingersoll et al., Status of Preconceptual Design of the Advanced High-Temperature Reactor (AHTR), ORNL/TM-2004/104, Oak Ridge National Laboratory, May 2004, pp. 46.

22. Decay Heat Power in Light Water Reactors, ANSI/ANS-5.1-2005, American Nuclear Society, LaGrange Park, Illinois, 2005.

23. A. A. Kulkarni, V. V. Ranade, R. Rajeev, and S. B. Koganti, "CFD Simulations of Flow in Vortex Diodes," Journal of American Institute of Chemical Engineering (AIChE), 54(5), 1139-1152 (March 2008).

24. H. Rothfuss and F. Vogt, "Reactor Vessel System," Nuclear Technology, 78, 245-254 (September 1987).

25. P. G. Smith, "High-Temperature Molten-Salt Lubricated Hydrodynamic Journal Bearings," ASLE Trans., 4(2): 263-274 (November 1961).

26. D. L. Barth, J. E. Pacheco, W. J. Kolb, and E. E. Rush, "Development of a High-Temperature, Long-Shafted, Molten-Salt Pump for Power Tower Applications," Proceedings of Solar Forum 2001: Solar Energy: The Power to Choose, April 21-25, 2001, Washington, D.C., American Society of Mechanical Engineers, 2001.

27. R. Flitney, Seals and Sealing Handbook, $5^{\text {th }}$ Edition, Elsevier, 2007.

28. R. E. MacPherson, Gas Cooled, Molten Salt Heat Exchanger-Design Study, ORNL-2605, Oak Ridge National Laboratory, October 1958.

29. K. Natesan, A. Moisseytsev, S. Majumdar, and P. S. Shankar, Preliminary Issues Associated with the Next Generation Nuclear Plant Intermediate Heat Exchanger Design, ANL/EXT06/46, Argonne National Laboratory, September 2006, pp. 97-106. 
30. R. B. Lindauer, Processing of the MSRE Flush and Fuel Salts, ORNL-TM-2578, Oak Ridge National Laboratory, August 1969.

31. T. T. Meek, Q. Han, and H. Xu, Degassing of Aluminum Alloys Using Ultrasonic Vibration, ORNL/TM-2006/61, Oak Ridge National Laboratory, 2006.

32. S. Fukada, Y. Maeda, M. Kinoshita, and T. Muroga, "Verification to Recover Tritium in Neutron-Irradiated Li by Y Plate," Fusion Engineering and Design, 82, 2152-2157 (2007).

33. A. N. Smith, Experience with Sodium Fluoroborate Circulation in an MSRE-Scale Facility, ORNL/TM-3344, Oak Ridge National Laboratory, January 1972.

34. Status of Liquid Metal Cooled Fast Reactor Technology, IAEA-TECDOC--1083, International Atomic Energy Agency, Vienna, 1999.

35. L. J. Koch et al., Hazards Summary Report, EBR-II, ANL-5719, Argonne National Laboratory, May 1957.

36. E. E. Feldman et al., "EBR-TJ Unprotected Loss-of-Heat-Sink Predictions and Preliminary Test Results," Nuclear Engineering and Design, 101, 57-66 (1987).

37. E. D. Throm, J. N. Wilson, R. R. Landry, and T. L. King, Preapplication Safety Evaluation Report for the Sodium Advanced Fast Reactor (SAFR) Liquid-Metal Reactor, NUREG-1369, U.S. Nuclear Regulatory Commission, December 1991.

38. G. Petrangeli, Nuclear Safety, Elsevier Butterworth-Heinemann, Burlington, Massachusetts, 2006, p. 30.

39. D. L. Barth, "High Temperature Molten Salt Pumps," Pumps \& Systems, pp. 98-101 (April 2007); available at www.pump-zone.com.

40. D. C. Smith, E. E. Rush, C. W. Matthews, J. M. Chavez, and P. A. Bator, Report on the Test of the Molten-Salt Pump and Valve Loops, SAND-91-1747, February 1992. 


\section{INTERNAL DISTRIBUTION}

1. J. T. Busby (busbyjt@,ornl.gov)

2. M. S. Cetiner (cetinerms@ornl.gov)

3. W. R. Corwin (corwinwr@ornl.gov)

4. G. F. Flanagan (flanagangf@ornl.gov)

5. J. C. Gehin (gehinjc@ornl.gov)

6. S. R. Greene (greenesr@ornl.gov)

7. D. E. Holcomb (holcombde@ornl.gov)

8. D. T. Ingersoll (ingersolldt@ornl.gov)

9. G. T. Mays (maysgt@ornl.gov)
10. C. V. Parks (parkscv@ornl.gov)

11. F. J. Peretz (peretzfj@ornl.gov

12. J. E. Rushton (rushtonje@ornl.gov)

13. J. C. Wagner (wagnerjc@ornl.gov)

14. D. F. Williams (williamsdf2@ornl.gov)

15. G. L. Yoder, Jr. (yodergljr@ornl.gov)

16. ORNL Laboratory Records-RC (hamrindr@,ornl.gov)

\section{EXTERNAL DISTRIBUTION}

17. M. H. Anderson, University of Wisconsin, 737 Engineering Research Building, 1500 Engineering Drive, Madison, WI 53706 (manderson@engr.wisc.edu)

18. C. W. Forsberg, Massachusetts Institute of Technology, MIT Nuclear Fuel Cycle Study Department of Nuclear Science and Engineering, 77 Massachusetts Ave., Room 24-207a, Cambridge, MA 02139 (cforsber@mit.edu)

19. T. O'Connor, NE-20/Germantown Building, U.S. Department of Energy, 1000 Independence Ave., SW., Washington, DC 20585-1290 (tom.oconnor@nuclear.energy.gov)

20. P. F. Peterson, University of California-Berkeley, Nuclear Engineering Department, 4153 Etcheverry Hall, Berkeley, CA 94720-1730 (Peterson@nuc.berkeley.edu)

21. P. S. Pickard, Sandia National Laboratory, P. O. Box 5800, Albuquerque, NM 87185-1136 (pspicka@sandia.gov)

22. R. D. Scheele, Pacific Northwest National Laboratory, P. O. Box 999, Richland, WA 99352 (randall.scheele@pnl.gov)

23. R. M.Versluis, NE-20/Germantown Building, U.S. Department of Energy, 1000 Independence Ave., S.W., Washington, DC 20585-1290 (rob.versluis@hq.doe.gov) 
